\title{
Front Matter: Volume 6968
}

, "Front Matter: Volume 6968," Proc. SPIE 6968, Signal Processing, Sensor Fusion, and Target Recognition XVII, 696801 (12 May 2008); doi:

10.1117/12.802000

SPIE Event: SPIE Defense and Security Symposium, 2008, Orlando, Florida, SPIE. United States 


\section{PROCEEDINGS OF SPIE}

\section{Signal Processing, Sensor Fusion, and Target Recognition XVII}

\section{Ivan Kadar}

Editor

17-19 March 2008

Orlando, Florida, USA

Sponsored and Published by

SPIE

Volume 6968 
The papers included in this volume were part of the technical conference cited on the cover and title page. Papers were selected and subject to review by the editors and conference program committee. Some conference presentations may not be available for publication. The papers published in these proceedings reflect the work and thoughts of the authors and are published herein as submitted. The publisher is not responsible for the validity of the information or for any outcomes resulting from reliance thereon.

Please use the following format to cite material from this book:

Author(s), "Title of Paper," in Signal Processing, Sensor Fusion, and Target Recognition XVII, edited by Ivan Kadar, Proceedings of SPIE Vol. 6968 (SPIE, Bellingham, WA, 2008) Article CID Number.

ISSN 0277-786X

ISBN 9780819471598

Published by

SPIE

P.O. Box 10, Bellingham, Washington 98227-0010 USA

Telephone +1 3606763290 (Pacific Time) · Fax +1 3606471445

SPIE.org

Copyright (C 2008, Society of Photo-Optical Instrumentation Engineers

Copying of material in this book for internal or personal use, or for the internal or personal use of specific clients, beyond the fair use provisions granted by the U.S. Copyright Law is authorized by SPIE subject to payment of copying fees. The Transactional Reporting Service base fee for this volume is $\$ 18.00$ per article (or portion thereof), which should be paid directly to the Copyright Clearance Center (CCC), 222 Rosewood Drive, Danvers, MA 01923. Payment may also be made electronically through CCC Online at copyright.com. Other copying for republication, resale, advertising or promotion, or any form of systematic or multiple reproduction of any material in this book is prohibited except with permission in writing from the publisher. The CCC fee code is $0277-786 \mathrm{X} / 08 / \$ 18.00$.

Printed in the United States of America.

Publication of record for individual papers is online in the SPIE Digital Library.

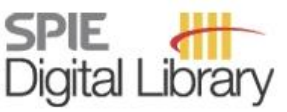

SPIEDigitallibrary.org

Paper Numbering: Proceedings of SPIE follow an e-First publication model, with papers published first online and then in print and on CD-ROM. Papers are published as they are submitted and meet publication criteria. A unique, consistent, permanent citation identifier (CID) number is assigned to each article at the time of the first publication. Utilization of CIDs allows articles to be fully citable as soon they are published online, and connects the same identifier to all online, print, and electronic versions of the publication. SPIE uses a six-digit CID article numbering system in which:

- The first four digits correspond to the SPIE volume number.

- The last two digits indicate publication order within the volume using a Base 36 numbering system employing both numerals and letters. These two-number sets start with 00, 01, 02, 03, 04, 05, $06,07,08,09,0 A, 0 B \ldots 0 Z$, followed by $10-12,20-2 Z$, etc.

The CID number appears on each page of the manuscript. The complete citation is used on the first page, and an abbreviated version on subsequent pages. Numbers in the index correspond to the last two digits of the six-digit CID number. 


\section{Contents}

ix Conference Committee

xiii Invited Panel Discussion: Issues and Challenges in Performance Assessment of Multitarget Tracking Algorithms with Applications to Real-World Problems

I. Kadar, Interlink Systems Sciences, Inc. (USA); E. Blasch, Air Force Research Lab. (USA);

C. Yang, Sigtem Technology, Inc. (USA); O. E. Drummond, CyberRnD, Inc. (USA) and Consulting Engineer (USA); W. D. Blair, Georgia Institute of Technology (USA); G. Chen, DCM Research Resources, LLC (USA); L. Bai, Temple Univ. (USA); C.-Y. Chong, BAE Systems (USA); X. R. Li, Univ. of New Orleans (USA); R. Mahler, Lockheed Martin MS2 Tactical Systems (USA); T. Kirubarajan, McMaster Univ. (Canada)

\section{MULTISENSOR FUSION, MULTITARGET TRACKING, AND RESOURCE MANAGEMENT I}

696802 Some interesting observations regarding the initialization of unscented and extended Kalman filters [6968-01]

A. J. Noushin, F. E. Daum, Raytheon Integrated Defense Systems (USA)

696804 A simple algorithm for sensor fusion using spatial voting (unsupervised object grouping) [6968-68]

H. M. Jaenisch, Alabama A\&M Univ. (USA) and Licht Strahl Engineering Inc. (USA);

N. G. Albritton, Amtec Corp. (USA); J. W. Handley, Licht Strahl Engineering Inc. (USA) and Amtec Corp. (USA); R. B. Burnett, R. W. Caspers, W. P. Albritton, Jr., Amtec Corp. (USA)

696805 A particle-filtering approach to convoy tracking in the midst of civilian traffic [6968-03] E. Pollard, B. Pannetier, ONERA (France); M. Rombaut, GIPSA Lab. (France)

696806 Long-term ground movement prediction by Monte Carlo simulation [6968-04]

M. J. Carlotto, General Dynamics Advanced Information Systems (USA)

696807 Gaussian mixture probability hypothesis density smoothing with multistatic sonar [6968-05] N. Nandakumaran, R. Tharmarasa, McMaster Univ. (Canada); T. Lang, General Dynamics Canada (Canada); T. Kirubarajan, McMaster Univ. (Canada)

696808 Estimating target range-Doppler image slope for maneuver indication [6968-06]

C. Yang, Sigtem Technology, Inc. (USA); E. Blasch, Air Force Research Lab. (USA)

696809 Effect of sensor bias on space-based bearing-only tracker [6968-07]

T. M. Clemons III, K. C. Chang, George Mason Univ. (USA)

MULTISENSOR FUSION, MULTITARGET TRACKING, AND RESOURCE MANAGEMENT II

6968 OA Improving automatic cooperation between UAVs through co-evolution [6968-08]

J. F. Smith III, Naval Research Lab. (USA) 
6968 OD Evaluation of an information-based sensor management system [6968-11]

J. Malachowski, K. J. Hintz, George Mason Univ. (USA)

$6968 \mathrm{OE} \quad$ Collaborative distributed sensor management and information exchange flow control for multitarget tracking using Markov decision processes [6968-12]

D. Akselrod, T. Kirubarajan, McMaster Univ. (Canada)

\section{ASSISTED TARGET RECOGNITION (ATR) I}

6968 OF Experimental feature-based SAR ATR performance evaluation under different operational conditions [6968-13]

Y. Chen, Intelligent Automation, Inc. (USA); E. Blasch, Air Force Research Lab. (USA);

H. Chen, Univ. of New Orleans (USA); T. Qian, Intelligent Automation, Inc. (USA); G. Chen, DCM Research Resources LLC (USA)

6968 OG Detecting missile-like flying target from a distance in sequence images [6968-14] X. Li, G. Chen, DCM Research Resources LLC (USA); E. Blasch, K. Pham, Air Force Research Lab. (USA)

$6968 \mathrm{OH} \quad$ Generation of efficient 2D templates from 3D multisensor data for correlation-based target tracking [6968-15]

C. Witte, W. Armbruster, K. Jäger, M. Hebel, Research Institute for Optronics and Pattern Recognition (Germany)

$69680 \mathrm{Ol}$ An approach for evaluating assisted target detection technology [6968-16] J. M. Irvine, National Geospatial-Intelligence Agency (USA); J. Leonard, Air Force Research Lab. (USA); P. Doucette, A. Martin, National Geospatial-Intelligency Agency (USA)

\section{ASSISTED TARGET RECOGNITION (ATR) II}

$69680 \mathrm{~J}$ On the limits of target recognition in the presence of atmospheric effects [6968-17]

X. Chen, N. A. Schmid, West Virginia Univ. (USA)

6968 OK A distributed automatic target recognition system using multiple low resolution sensors [6968-18]

Z. Yue, P. Lakshmi Narasimha, P. Topiwala, FastVDO Inc. (USA)

$6968 \mathrm{OL} \quad$ Multi-viewpoint image fusion for urban sensing applications [6968-19]

F. Ahmad, M. G. Amin, Villanova Univ. (USA)

$69680 \mathrm{M}$ Virtual simulation tools for artillery [6968-20]

P. Gozard, E. Bret, DGA/DET/ETBS (France); T. Cathala, OKTAL Synthetic Environment (France)

\section{MULTISENSOR FUSION METHODOLOGIES AND APPLICATIONS I}

$69680 \mathrm{~N} \quad$ Multitarget-moment filters for nonstandard measurement models [6968-21]

R. Mahler, Lockheed Martin MS2 Tactical Systems (USA) 
696800 Joint search and sensor management for geosynchronous satellites [6968-22] A. Zatezalo, A. El-Fallah, Scientific Systems Co., Inc. (USA); R. Mahler, Lockheed Martin Tactical Defense Systems (USA); R. K. Mehra, Scientific Systems Co., Inc. (USA); K. Pham, Air Force Research Lab. (USA)

6968 OP Dynamic sensor management of dispersed and disparate sensors for tracking resident space objects [6968-23]

A. El-Fallah, A. Zatezalo, Scientific Systems Co., Inc. (USA); R. Mahler, Lockheed Martin Tactical Defense Systems (USA); R. K. Mehra, Scientific Systems Co., Inc. (USA); D. Donatelli, Air Force Research Lab. (USA)

\section{MULTISENSOR FUSION METHODOLOGIES AND APPLICATIONS II}

$69680 Q$ An initial investigation into incorporating human reports into a road-constrained random set tracker [6968-31]

D. W. Winters, J. B. Witkoskie, W. S. Kuklinski, The MITRE Corp. (USA)

6968 OR Service-based extensions to the JDL fusion model [6968-25]

R. T. Antony, Science Applications International Corp. (USA); J. A. Karakowski, U.S. Army RDEC (USA)

6968 OS Handling target obscuration through Markov chain observations [6968-26] M. A. Kouritzin, B. Wu, Univ. of Alberta (Canada)

\section{MULTISENSOR FUSION METHODOLOGIES AND APPLICATIONS III}

6968 OT Statistical, biological, and categorical sensor fusion: an integrated methodology [6968-27]

J. Bonick, C. Marshall, U.S. Army RDECOM CERDEC NVESD (USA)

6968 OU A confidence paradigm for classification systems [6968-28]

N. J. Leap, K. W. Bauer, Jr., Air Force Institute of Technology (USA)

\section{MULTISENSOR FUSION METHODOLOGIES AND APPLICATIONS IV}

6968 0X Structured pedigree information for distributed fusion systems [6968-24]

P. O. Arambel, BAE Systems (USA)

6968 OY Analytical performance evaluation for autonomous sensor fusion [6968-32]

K. C. Chang, George Mason Univ. (USA)

$69680 Z$ Convergence study of message passing in arbitrary continuous Bayesian networks [6968-33]

W. Sun, K. C. Chang, George Mason Univ. (USA)

$696810 \quad$ Algebra of Dempster-Shafer evidence accumulation [6968-34]

R. H. Enders, A. K. Brodzik, M. R. Pellegrini, The MITRE Corp. (USA) 
696811 Incorporation of indirect evidence into an evidence accrual technique for higher level data fusion [6968-36]

S. C. Stubberud, Rockwell-Collins (USA); K. A. Kramer, Univ. of San Diego (USA)

696812 Results from levels 2/3 fusion implementations: issues, challenges, retrospectives, and perspectives for the future an annotated perspective (Invited Paper) [6968-37]

I. Kadar, Interlink Systems Sciences, Inc. (USA); E. Bosse, Defence R\&D Canada, Valcartier (Canada); J. Salerno, Air Force Research Lab. (USA); D. A. Lambert, Defence Science and Technology Organisation (Australia); S. Das, Charles River Analytics, Inc. (USA); E. H. Ruspini, SRI International (USA); B. J. Rhodes, BAE Systems (USA); J. Biermann, FGAN-FKIE (Germany)

696813 A collaborative eye to the future [6968-38]

K. A. Greene, T. Goan, E. R. Creswick, Stottler-Henke Associates, Inc. (USA)

\section{SIGNAL AND IMAGE PROCESSING I}

696814 Efficient super-resolution image reconstruction applied to surveillance video captured by small unmanned aircraft systems [6968-39]

Q. He, Mississippi Valley State Univ. (USA); R. R. Schultz, Univ. of North Dakota (USA);

C.-H. H. Chu, Univ. of Lovisiana at Lafayette (USA)

696815 Super-resolution image reconstruction from UAS surveillance video through affine invariant interest point-based motion estimation [6968-40]

Q. He, Mississippi Valley State Univ. (USA); R. R. Schultz, A. Camargo, Y. Wang, F. Martel, Univ. of North Dakota (USA)

696816 A smart iterative algorithm for multisensor image registration [6968-41]

S. DelMarco, V. Tom, H. Webb, BAE Systems (USA)

696817 3D organization of 2D urban imagery [6968-42]

P. Cho, Massachusetts Institute of Technology (USA)

696818 Fusing images and maps [6968-43]

M. J. Carlotto, General Dynamics Advanced Information System (USA)

696819 An investigation of image fusion algorithms using a visual performance-based image evaluation methodology [6968-44]

K. E. Neriani, Consortium Research Fellows Program (USA) and Air Force Research Lab. (USA); A. R. Pinkus, Air Force Research Lab. (USA); D. W. Dommett, General DynamicsAdvanced Information Engineering Systems (USA)

$69681 \mathrm{~A}$ Compressed sensing technique for high-resolution radar imaging [6968-45]

Y.-S. Yoon, M. G. Amin, Villanova Univ. (USA)

6968 1C Fast algorithms for video target detection and tracking [6968-47]

C. Li, J. Si, Arizona State Univ. (USA); G. P. Abousleman, General Dynamics C4 Systems (USA) 
6968 1D Interval least-squares filtering with applications to video target tracking [6968-48] B. Li, C. Li, J. Si, Arizona State Univ. (USA); G. Abousleman, General Dynamics C4 Systems (USA)

SIGNAL AND IMAGE PROCESSING II

6968 IE Frustrated polarization fiber Sagnac interferometer displacement sensor [6968-50]

A. D. McAulay, J. Wang, Lehigh Univ. (USA)

$6968 \mathrm{IF} \quad$ On modeling sea clutter by diffusive models [6968-51]

J. Hu, Univ. of Florida (USA); W. Tung, Purdue Univ. (USA); J. Gao, Univ. of Florida (USA);

R. S. Lynch, Naval Undersea Warfare Ctr. (USA); G. Chen, DCM Research Resources LLC (USA)

6968 1G On modeling sea clutter by noisy chaotic dynamics [6968-52]

W. Tung, Purdue Univ. (USA); J. Hu, J. Gao, Univ. of Florida (USA); R. S. Lynch, Naval Undersea Warfare Ctr. (USA); G. Chen, DCM Research Resources LLC (USA)

$69681 \mathrm{~J}$ Large spot size laser vibrometry insensitivity occurs in 1-D vibrations only [6968-58]

M. C. Kobold, General Dynamics-Advanced Information Systems (USA)

SIGNAL AND IMAGE PROCESSING III

$6968 \mathrm{lK}$ The use of interferoceiver for the prevention of fratricide in missile defense [6968-55]

M.-C. Li, Liceimer (USA)

$69681 \mathrm{M}$ Empirical performance of the spectral independent morphological adaptive classifier [6968-57]

J. B. Montgomery, C. T. Montgomery, M\&M Aviation (USA); R. B. Sanderson,

J. F. McCalmont, Air Force Research Lab. (USA)

$69681 \mathrm{~N}$ Comparison of theoretical and empirical statistics of wind measurements with validation lidar (VALIDAR) [6968-59]

J. Y. Beyon, California State Univ., Los Angeles (USA); G. J. Koch, M. J. Kavaya, NASA

Langley Research Ctr. (USA); M. Sahota, California State Univ., Los Angeles (USA)

\section{POSTER SESSION}

696810 A real-time geographical information exchange system with PDA [6968-60]

J. He, X. Zhang, B. Sun, Beijing Normal Univ. (China)

6968 iP Object recognition in infrared image sequences using scale invariant feature transform [6968-61]

C. Park, K. Bae, Samsung Thales Co., Ltd. (South Korea); J.-H. Jung, Korea Advanced Institute of Science and Technology (South Korea) 
$69681 Q \quad$ Image fusion in infrared image and visual image using normalized mutual information [6968-62]

C. Park, K. Bae, S. Choi, Samsung Thales Co., Ltd. (South Korea); J.-H. Jung, Korea Advanced Institute of Science and Technology (South Korea)

6968 IR Dynamic stimulus enhancement with Gabor-based filtered images [6968-63]

A. R. Pinkus, Air Force Research Lab. (USA); M. J. Poteet, Consortium Research Fellows Program (USA); A. J. Pantle, Miami Univ. (USA)

6968 is Correlating military operators' visual demands with multi-spectral image fusion [6968-64] G. L. Martinsen, J. S. Hosket, A. R. Pinkus, Air Force Research Lab. (USA)

$69681 \mathrm{U}$ Multisensory data exploitation using advanced image fusion and adaptive colorization [6968-66]

Y. Zheng, K. Agyepong, O. Kuljaca, Alcorn State Univ. (USA)

6968 IV Multi-mode sensor processing on a dynamically reconfigurable massively parallel processor array [6968-67]

P. Chen, M. Butts, B. Budlong, P. Wasson, Ambric, Inc. (USA)

Author Index 


\title{
Conference Committee
}

\author{
Symposium Chair
}

Larry B. Stotts, Defense Advanced Research Projects Agency (USA)

Symposium Cochair

Ray O. Johnson, Lockheed Martin Corporation (USA)

Program Track Chair

Ivan Kadar, Interlink Systems Sciences, Inc. (USA)

Conference Chair

Ivan Kadar, Interlink Systems Sciences, Inc. (USA)

Program Committee

Mark G. Alford, Air Force Research Laboratory (USA)

William D. Blair, Georgia Tech Research Institute (USA)

Erik Blasch, Air Force Research Laboratory (USA)

Mark J. Carlotto, General Dynamics Corporation (USA)

Kuo-Chu Chang, George Mason University (USA)

Chee-Yee Chong, BAE Systems Advanced Information Technologies (USA)

Marvin N. Cohen, Georgia Tech Research Institute (USA)

Mohammad Farooq, Royal Military College of Canada (Canada)

Charles W. Glover, Oak Ridge National Laboratory (USA)

I. R. Goodman, Space and Naval Warfare Systems Center, San Diego (USA)

Lynne L. Grewe, California State University/East Bay (USA)

Michael L. Hinman, Air Force Research Laboratory (USA)

Kenneth J. Hintz, George Mason University (USA)

Jon S. Jones, Air Force Research Laboratory (USA)

Thiagalingam Kirubarajan, McMaster University (Canada)

Martin E. Liggins II, MITRE Corporation (USA)

Perry C. Lindberg, Teledyne Brown Engineering (USA)

James Llinas, University at Buffalo (USA)

Ronald P. Mahler, Lockheed Martin Company/Tactical Systems (USA)

Raj P. Malhotra, Air Force Research Laboratory (USA)

Alastair D. McAulay, Lehigh University (USA)

Raman K. Mehra, Scientific Systems Company, Inc. (USA)

Harley R. Myler, Lamar University (USA)

David Nicholson, BAE Systems plc (United Kingdom) 
Leslie M. Novak, BAE Systems Advanced Information Technologies (USA)

Andrew G. Tescher, AGT Associates (USA)

Stelios C. A. Thomopoulos, National Center for Scientific Research (Greece)

Wiley E. Thompson, New Mexico State University (USA)

Session Chairs

Multisensor Fusion, Multitarget Tracking, and Resource Management I

Ivan Kadar, Interlink Systems Sciences, Inc. (USA)

Thiagalingam Kirubarajan, McMaster University (Canada)

Kenneth J. Hintz, George Mason University (USA)

Multisensor Fusion, Multitarget Tracking, and Resource Management II

Kenneth J. Hintz, George Mason University (USA)

Ivan Kadar, Interlink Systems Sciences, Inc. (USA)

Assisted Target Recognition (ATR) I

Ivan Kadar, Interlink Systems Sciences, Inc. (USA)

Kenneth J. Hintz, George Mason University (USA)

Assisted Target Recognition (ATR) II

Ivan Kadar, Interlink Systems Sciences, Inc. (USA)

Kenneth J. Hintz, George Mason University (USA)

Multisensor Fusion Methodologies and Applications I

Ronald P. Mahler, Lockheed Martin Corporation (USA)

Multisensor Fusion Methodologies and Applications II

Ronald P. Mahler, Lockheed Martin Corporation (USA)

Multisensor Fusion Methodologies and Applications III

Michael L. Hinman, Air Force Research Laboratory (USA)

Chee-Yee Chong, BAE Systems Advanced Information Technologies (USA)

Ivan Kadar, Interlink Systems Sciences, Inc. (USA)

Multisensor Fusion Methodologies and Applications IV

Michael L. Hinman, Air Force Research Laboratory (USA)

Chee-Yee Chong, BAE Systems Advanced Information Technologies (USA)

Ivan Kadar, Interlink Systems Sciences, Inc. (USA) 
Multisensor Fusion Methodologies and Applications $V$

Michael L. Hinman, Air Force Research Laboratory (USA)

Ivan Kadar, Interlink Systems Sciences, Inc. (USA)

Signal and Image Processing I

Lynne L. Grewe, California State University/East Bay (USA)

Alastair D. McAulay, Lehigh University (USA)

Mark G. Alford, Air Force Research Laboratory (USA)

Signal and Image Processing II

Alastair D. McAulay, Lehigh University (USA)

Lynne L. Grewe, California State University/East Bay (USA)

Mark G. Alford, Air Force Research Laboratory (USA)

Signal and Image Processing III

Mark G. Alford, Air Force Research Laboratory (USA)

Lynne L. Grewe, California State University/East Bay (USA)

Alastair D. McAulay, Lehigh University (USA) 
Downloaded From: https://www.spiedigitallibrary.org/conference-proceedings-of-spie on 25 Apr 2023

Terms of Use: https://www.spiedigitallibrary.org/terms-of-use 


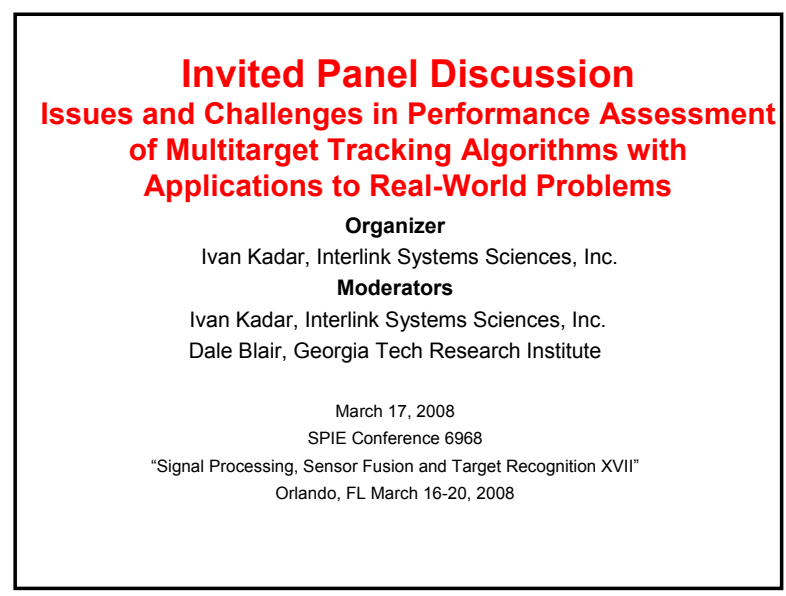

Invited Panel Discussion
Topics
"Issues and Challenges in Model-Based Performance
Assessment of Multi-Target Trackers" Dr. Ivan Kadar,
Interlink Systems Sciences, Inc.
"Target-to-Track Assignment in Performance Evaluation
of Multiple Target Tracking” Dr. Oliver Drummond P.E.,
CyberRnD, Inc., and Consulting Engineer
"Modeling and Simulation: Key to Effective Performance
Assessment of Multiple Tracking Algorithms "
Dr. Dale Blair, Georgia Tech Research Institute.
"Performance Assessment of Multitarget Tracking
Algorithms in the Real World“ Dr. Erik P. Blasch, Air
Force Research Lab., Dr.Chun Yang, Sigtem Technology,
Inc., Dr.Genshe Chen, DCM Research Resources, LLC,
Dr.Ivan Kadar, Interlink Systems Sciences, Inc., and
Professor Li Bai,Temple University

\section{Invited Panel Discussion \\ Participants:}

Dr. Dale Blair, Georgia Tech. Research Institute

Dr. Erik P. Blasch, Air Force Research Lab.

Dr. Chee-Yee Chong, BAE Systems

Dr. Oliver Drummond, CyberRnD and Consulting Engineer

Dr. Ivan Kadar, Interlink Systems Sciences, Inc.

Professor Thiagalingam Kirubarajan, McMaster Univ.

Professor X. Rong Li, Univ. of New Orleans

Dr. Ronald P. S. Mahler, Lockheed Martin MST2 Tactical Systems

Invited Panel Discussion
Topics
"Consumer Reports Approach for Assessing Multi-target
Trackers" Dr. Chee-Yee Chong, BAE Systems
"Challenges and Recent Advances in Estimation
Performance Evaluation" Professor X. Rong Li, Univ. of
New Orleans
"Global Performance MOEs for Multitarget Tracking"
Dr. Ronald P. S. Mahler, Lockheed Martin MS2 Tactical
Systems
"How Useful Are Theoretical Bounds in the Real World"
Professor Thiagalingam Kirubarajan, McMaster Univ.
(Canada)




\title{
Issues and challenges in model-based performance assessment of multi-target trackers
}

\author{
Ivan $\operatorname{Kadar}^{1}$, Erik Blasch ${ }^{2}$ and Chun Yang ${ }^{3}$ \\ ${ }^{1}$ Interlink Systems Sciences, Inc., 1979 Marcus Ave., STE 210, Lake Success, NY 11042 \\ ${ }^{2}$ AFRL/RYAA - Fusion Evaluation, 2241 Avionics Cir, WPAFB, OH 45433-7001 \\ ${ }^{3}$ Sigtem Technology, Inc., 1343 Parrott Drive, San Mateo, CA 94402
}

\begin{abstract}
This paper presents a basic look at the issues and challenges in assessing the performance of multi-target trackers using model-based simulation/prediction/correction methods. Issues of realistic modeling of "real data" including effects of target densities and target behaviors, environment (clutter) and sensor models are highlighted. The use of robust preprocessing is proposed to eliminate measurement outliers. The performance assessment consists of decomposing the tracker into association and state estimation elements, and comparing the performance of the individual components to the tracking system performance, with both simulated model-based and with real data. The potential of either post-hoc, and/or on-line performance-monitoring methods of the tracker under test are proposed along with corrective feedback in order to improve tracking system performance.
\end{abstract}

Keywords: Predictive/corrective performance assessment, multi-target tracking algorithms

\section{INTRODUCTION}

This paper presents a basic and succinct view of issues and challenges in assessing the performance of multi-target trackers using model-based simulation/prediction/correction methods. Issues of realistic modeling of "real data" including effects of target densities and target behaviors, environment (clutter) and sensor models are highlighted. The use of robust preprocessing is proposed to eliminate measurement outliers. Robust-preprocessing removes outliers that persist as propagating errors through the tracker, but does not mitigate the effects of non-Gaussian measurement noise caused by clutter and the multiplicative noise effects of multipath. The proposed performance assessment scheme consists of decomposing the tracker into association and prediction/state estimation elements, and comparing (1) the performance of the individual components over time, (2) tracking system performance over time, and (3) individual-tosystem performance, with both simulated model-based and real data. The potential of either post-hoc, and/or on-line methods of monitoring the performance of the tracker under test are proposed along with corrective feedback in order to improve tracking system performance, sensor [2] and environmental models.

The paper is organized as follows: we first depict methods of performance assessment, followed by the above proposed approach, and subsequently discuss models, methods, issues, and challenges in addressing the complexity of the problem. As system-level performance is challenged by increased complexity (e.g. distributed processing), it is ever important to understand the issues and methods in developing a comprehensive performance evaluation plan.

\section{MODEL-BASED PERFORMANCE ASSESSMENT: ISSUES AND CHALLENGES}

It is well known that there are two ways to predict an estimator kinematic performance [1], one is to use a model-based simulation approach, and other is to use an analytical approach. The model-based approach, both utilizes and combines environment (clutter), target behavior/characteristics, detection and sensor models [2], and uses a time series of simulated or real measurements to form a state estimate, which is compared with respect to a measure-of-merit [3] or ground truth. An analytical model, if feasible, would rely on a steady state filter model to predict the performance of the state estimate, given target, environment and sensor models. Furthermore, the analytical model, in general, assumes stationary target and sensor model parameters [2], and would not allow taking into account time dependence inherent in a tracking system. Note that the above discussion assumed perfect measurement-to-track association. To account for uncertainty in measurement-to-track association, an optimal association metric in terms of probability of correct association, $\mathrm{P}_{\mathrm{CA}}$, can be used to provide a probabilistic bound on the achievable association performance for a given scenario, as proposed by [4]. The probability of correct association is given by, $\mathrm{P}_{C A}=\mathrm{e}^{-\beta}$, where, $\beta$ is the normalized target density defined as the average number of objects in a one-sigma region of the measurement space, $D_{m}$, and $\beta$ is 
given by, $\beta=D_{m}|R|^{1 / 2}$, where $R$ is the measurement error covariance [4]. Typical association algorithms can consist of nearest neighbor, Jonker-Volgenant-Castonon (JVC) assignment, multi-frame association or multiple hypotheses tracking [5].

It is also well known [5, 6, 7], that trackers are intrinsically nonlinear, (even in the case of a linearized model, depending on the type of measurements and parametric excursions) and the measurement error sources can become potentially nonGaussian, and also can be contaminated with outliers. In spite of this, algorithms are usually designed under the usual Additive White Gaussian Noise (AWGN) assumption [5]. In addition, with many maneuvering or crossing and mergeand-split targets present, even under AWGN measurement noise conditions, the tracker may not be able to model all possible target dynamics, and the resultant distribution of the innovations sequence may not necessarily be AWG distributed [5]. While the later condition can be used to identify target maneuvers [5], it will also degrade tracker performance, and thus can become a metric [8] to be monitored for performance degradation as described in Section 3. In order to assess the efficacy of the models inherent in a given tracker, [9] proposed the maneuvering index, $\lambda$, as a measure to determine whether a single model or multiple interacting models are needed, depending of the value of $\lambda$, [5, 9] for best performance. The target maneuvering index, $\lambda$, is given by $\lambda=\sigma_{v} T^{2} / \sigma_{w}$, where $\sigma_{v}$ is the process noise standard deviation, $T$ is the revisit interval, and $\sigma_{\mathrm{w}}$ is the measurement noise standard deviation.

Therefore, depending on computation of specific range of values of $\lambda$ [9] for a given tracker/model, will give the modeler a-priori knowledge what performance to expect under certain stressing maneuvering target conditions before testing and, along with the computation of $\mathrm{P}_{\mathrm{CA}}$, aid the predictive and corrective performance assessment method discussed below.

\section{A MODEL-BASED PREDICTIVE AND CORRECTIVE-FEEDBACK METHOD}

Given the above description of the complex problem setting, the question arises how to evaluate tracker performance, based on performance goals, performance prediction (tracker model) and ground truth, especially since models may not be able to account for the effects of nonlinearities present in trackers. Figure 1 depicts a model-based predictive/corrective approach, consisting of both post-hoc (forensic) assessment and/or an on-line monitoring corrective feedback concept for performance assessment and enhancement.

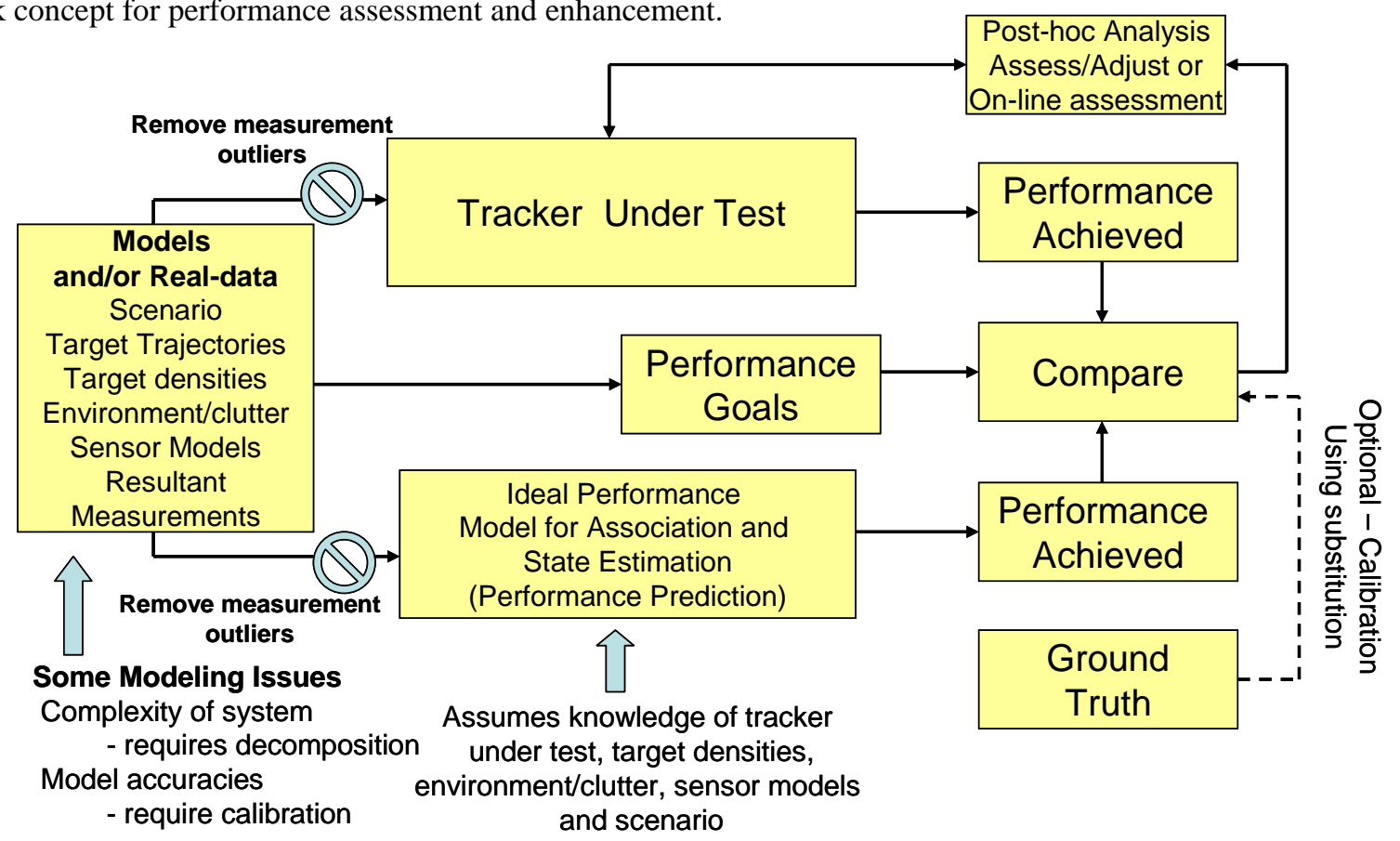

Figure 1. A model-based predictive and corrective performance assessment concept

In order to minimize potential nonlinear effects on performance due measurement noise outliers, the measurements are censored by robust nonparametric methods, such as Tukey's method of Hinges [8, 10], as indicated in Fig. 1. The 
robustized measurements serve as inputs to both the tracker under test, and to the ideal performance model of the tracker. Of course, there are many other error sources remaining, such as the effects of non-Gaussian measurement noise, and large parameter excursions in measurements, etc.

Performance metrics can be either absolute, with respect to ground truth, or using a substitution method to calibrate "predicted (i.e., model) performance" with respect to ground truth, or relative with respect to goals, refer to Fig. 1, as established by the scenario, by using selected families of tracking/estimation metrics as described in [ 5, 6, 7, 11].

In order to optimize tracker performance under test, two methods of corrective feedback are considered, refer to Fig. 1: (1) a post-hoc (forensic) analysis of tracker performance by monitoring the state estimates, and the distribution of the innovations sequence [8], (2) using on-line assessment of the same parameters as in (1), but providing on-line feedback to both the tracker dynamic models and gating to improve performance, as needed. In order to evaluate the tracker components, consisting of association and state estimation, a single target scenario or widely separated targets can be used to remove the effects of association, and to test the estimation component with a range of target motions/dynamics, sensor errors and update rates [7]. In an analogous manner, the association component can be tested with nonmaneuvering targets. It should be noted, that while not explicitly stated, the above method could be applied to evaluate families of trackers, e.g., using linearized and/or extended Kalman filters, and interacting multiple models, utilizing association algorithms such as nearest neighbor, JVC, multiple hypotheses tracking or multi-frame association [5].

\section{CONCLUSIONS}

This paper presented a basic and comprehensive view of issues and challenges in assessing the performance of multitarget trackers using model-based simulation/prediction/correction methods. Issues of realistic modeling of "real data" including effects of target densities and target behaviors, environment (clutter) and sensor models were highlighted. The use of robust preprocessing of measurements was proposed to eliminate measurement noise produced outliers. The proposed performance assessment consisted of decomposing the tracker into association and state estimation elements, in order compare the performance of the individual tracker components, and of the tracking system performance, with both simulated model-based and with real data. The potential of either post-hoc, and/or on-line methods of monitoring the performance of the tracker under test were proposed along with corrective feedback in order to improve tracking system performance. The next step is to test the efficacy of the proposed conceptual method first in a simulation testbed, as an extension to [8], and subsequently in a benchmark like environment [7] with real data.

\section{REFERENCES}

[1] Chang, K.C., Sivaraman, E., and Liggins, M, "Performance Modeling for Multisensor Tracking and Classification”, Proc. SPIE 5429, 335-342 (2004).

[2] Carlotto, M. and Kadar, I., "Multi-source Report-level Simulator for Fusion Research”, Proc. SPIE 5069, 364-378 (2003).

[3] Kadar, I., Chang, K. C., O’Connor, C., and Liggins, M., "Figures-of-Merit to bridge fusion, long term prediction and dynamic sensor management”, Proc. SPIE 5429, 343-349 (2004).

[4] Mori, S., Chang, K. C., and Chong, C-Y, "Performance Analysis of Optimal Data Association with Application to Multiple Target Tracking”, in Multitarget-Multisensor Tracking: Applications and Advances, Vol. II, Chapter 7, Edited by Y. Bar-Shalom, Artech House (1992).

[5] Bar-Shalom, Y., Li, X. R., and Kirubarajan, T., Estimation with Application to Tracking and Navigation, Artech House, (2001).

[6] Drummond, O. E., Blair, W. D., Brown, G. C., Ogle, T. L., Bar-Shalom, Y., Cooperman, R. L., and Barker, W. H., "Performance Assessment and Comparison of Various Tracklet Methods for Maneuvering Targets", Proc. SPIE 5096, 514-538 (2003).

[7] Blair, W.D., Watson, G. A., Kirubarajan, T., and Y. Bar-Shalom, Y., "Benchmark for Radar Resource Allocation and Tracking Targets In the Presence of ECM,” IEEE Trans. Aero. Elect. Sys., 1097-1114 (1998).

[8] Yang, C., Blasch, E. P. and Kadar, I. "Optimality Self Online Monitoring (OSOM) for Performance Evaluation and Adaptive Sensor Fusion”, to appear.

[9] Kirubarajan, T. and Bar-Shalom, Y., "Kalman Filter versus IMM Estimator: When do we need the later?,” IEEE Trans. Aero. Elect. Sys., 1452-1457 (2003).

[10] NIST/SEMATECH e-Handbook of Statistical Methods, http://www.itl.nist.gov/div898/handbook/

[11] Li, X. R. and Zhao, Z., "Relative Error Measures for Evaluation of Estimation Algorithms", Proc. Fusion05 (2005). 
Target-to-Track Assignment in Performance Evaluation of Multiple Target Tracking

Excerpts From

Excerpts From SPIE Panel on Issues and Challenges in Performance Assessment of
Multitarget Tracking Algorithms with Applications to Real-World Problems 17 March 2008

Oliver E. Drummond, Ph.D., P.E.

CyberRnD, Inc. and Consulting Engineer

Phone: 310-838-5300

Email: drummond@worldnet.att.net

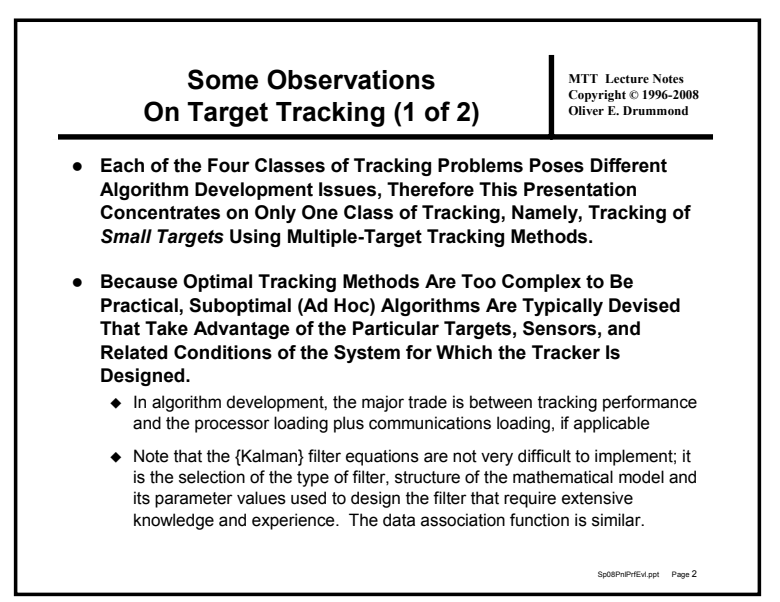

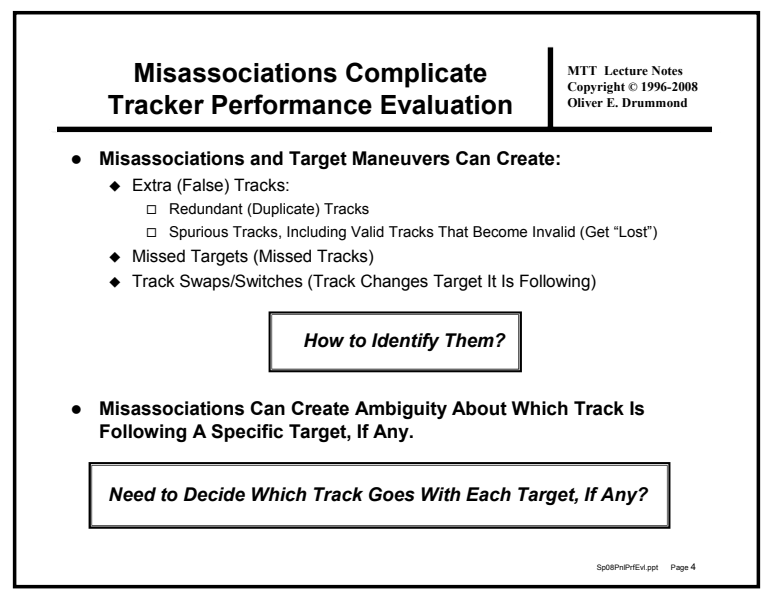
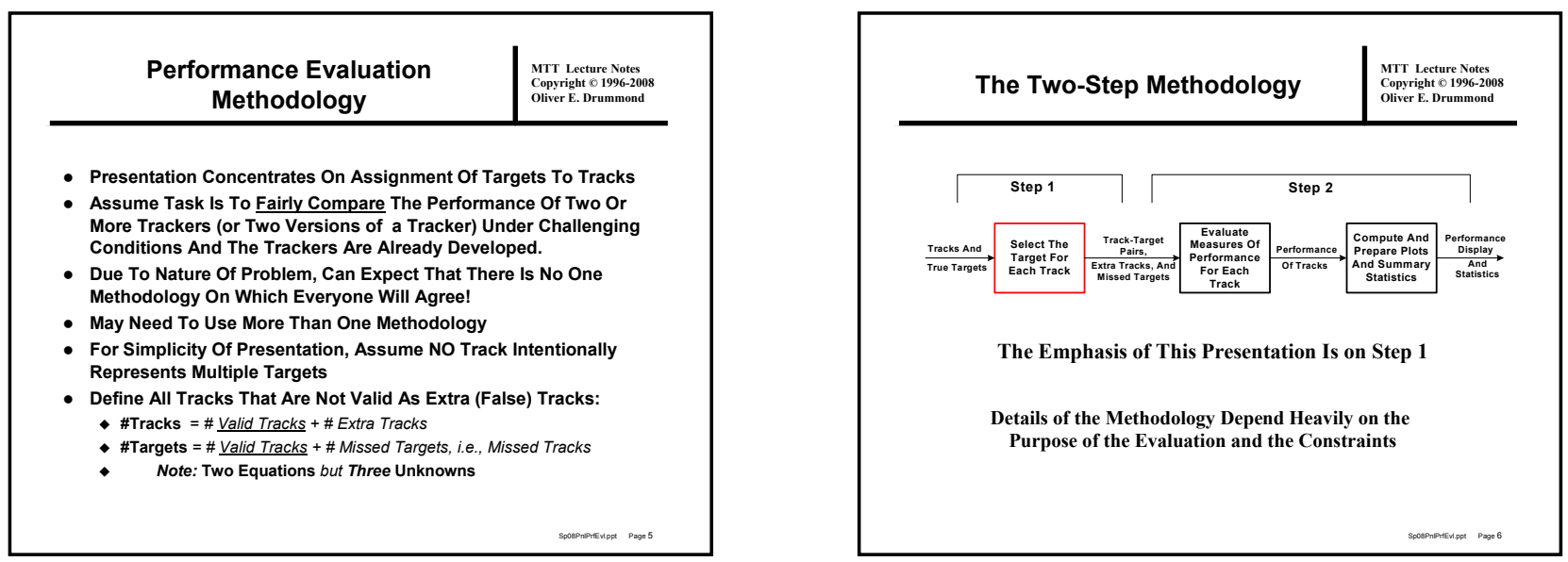

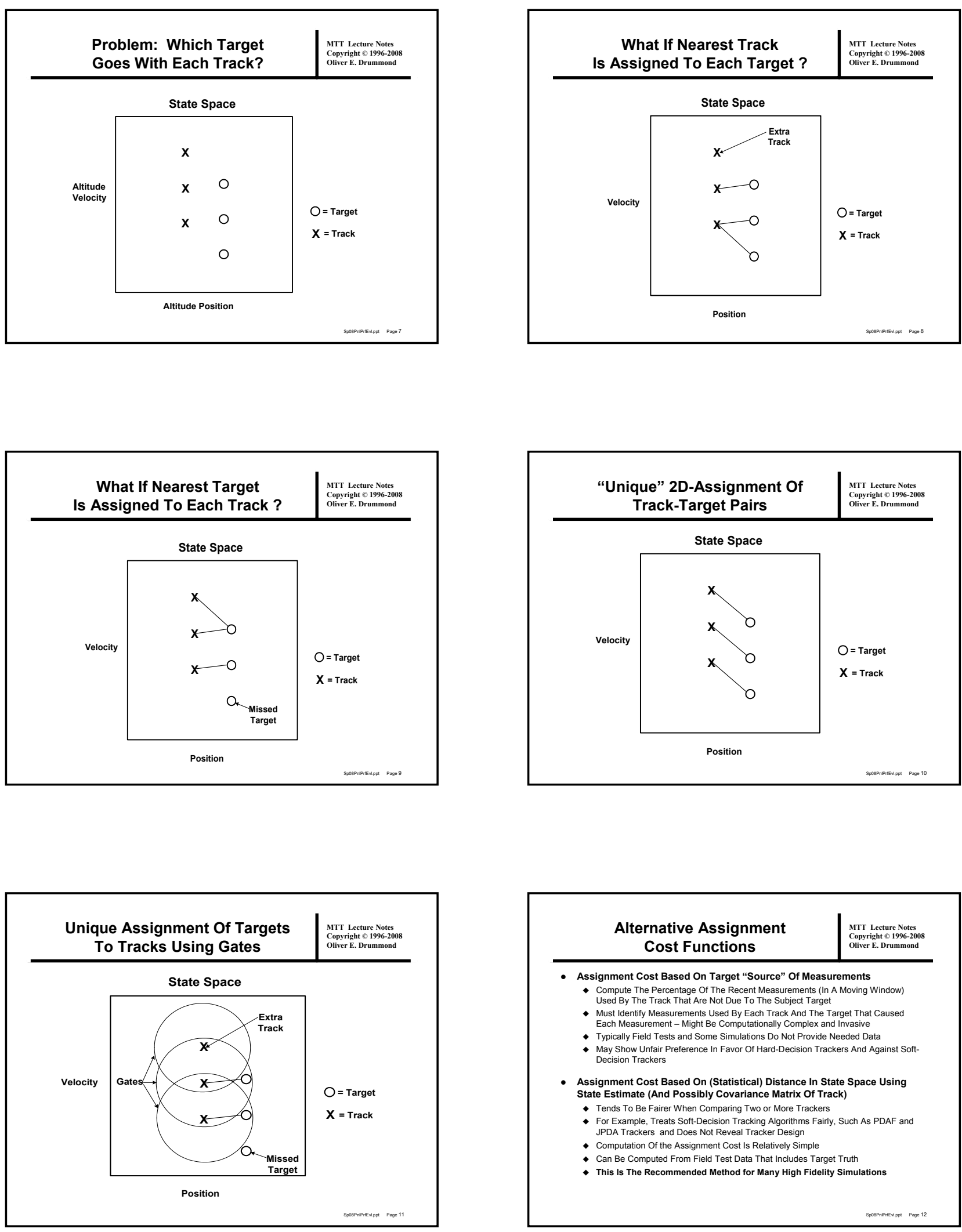

xviii 

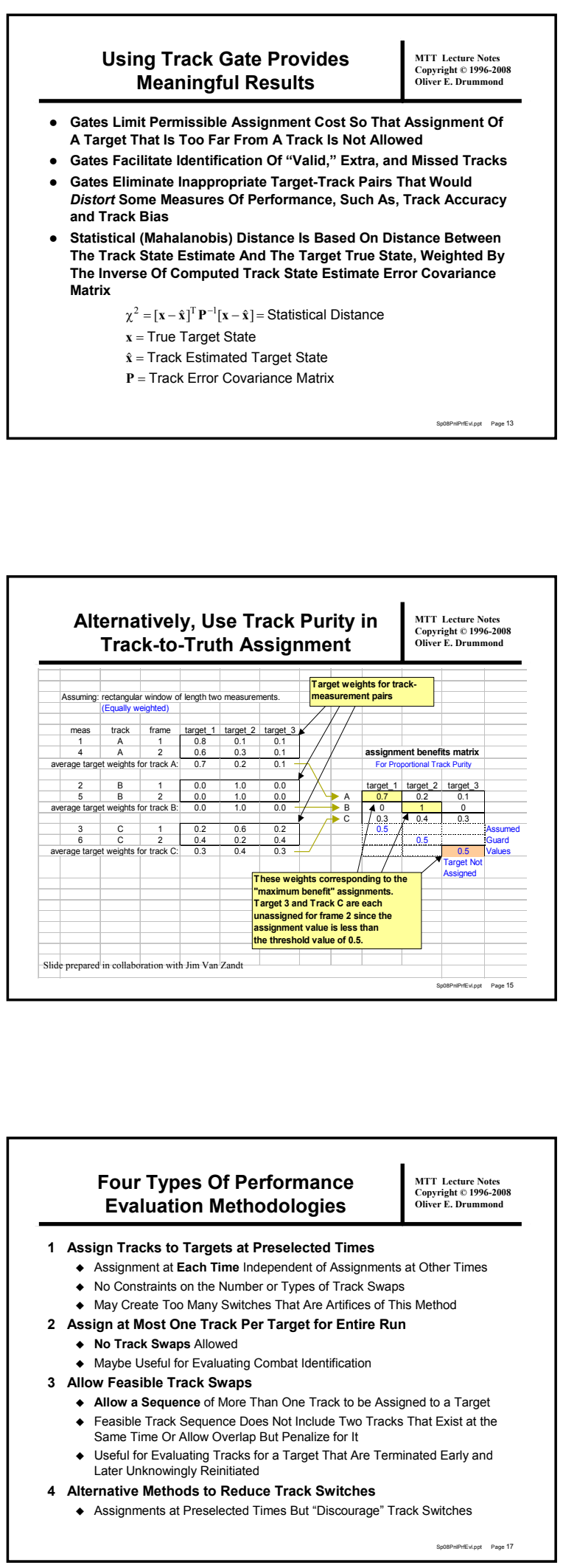

For High Fidelity Simulation Consider Using "Statistical Distance" And Gates To Uniquely Assign Targets To Tracks MTT Lecture Notes Copyright $C$ 1996-200
Oliver E. Drummond

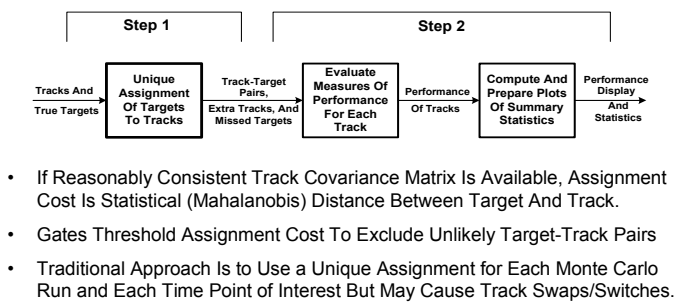

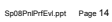

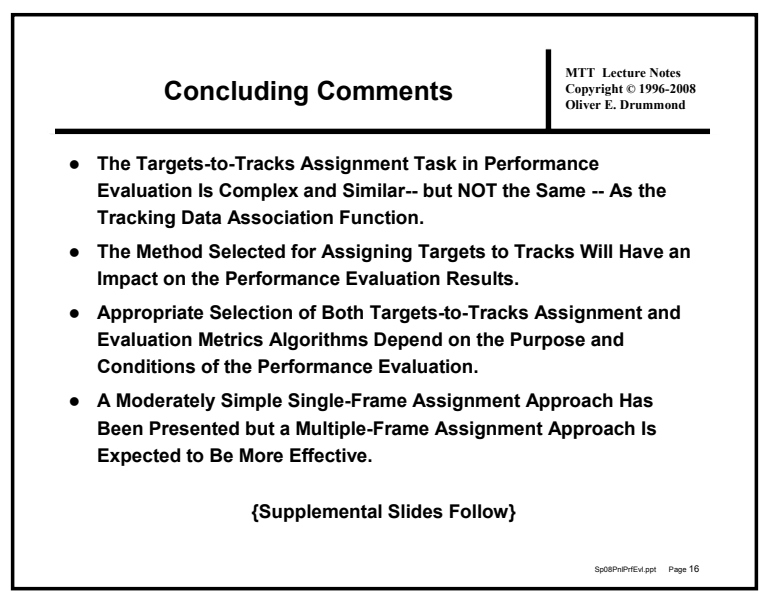

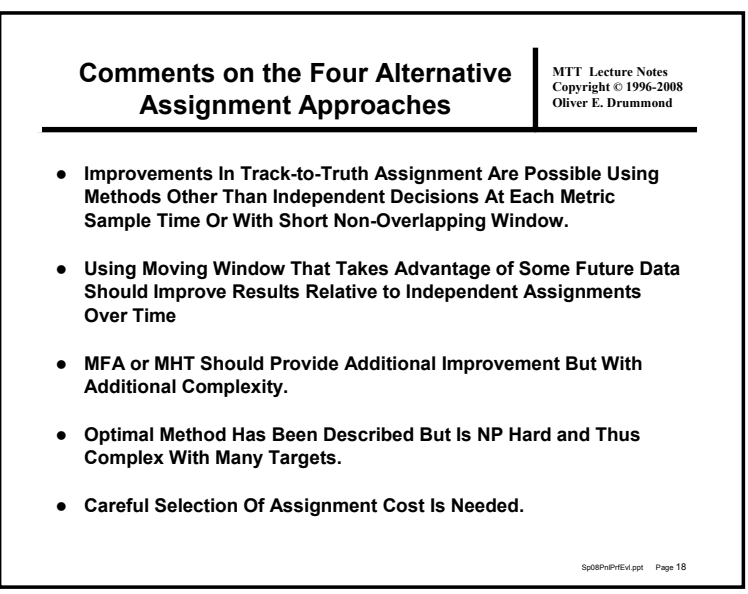



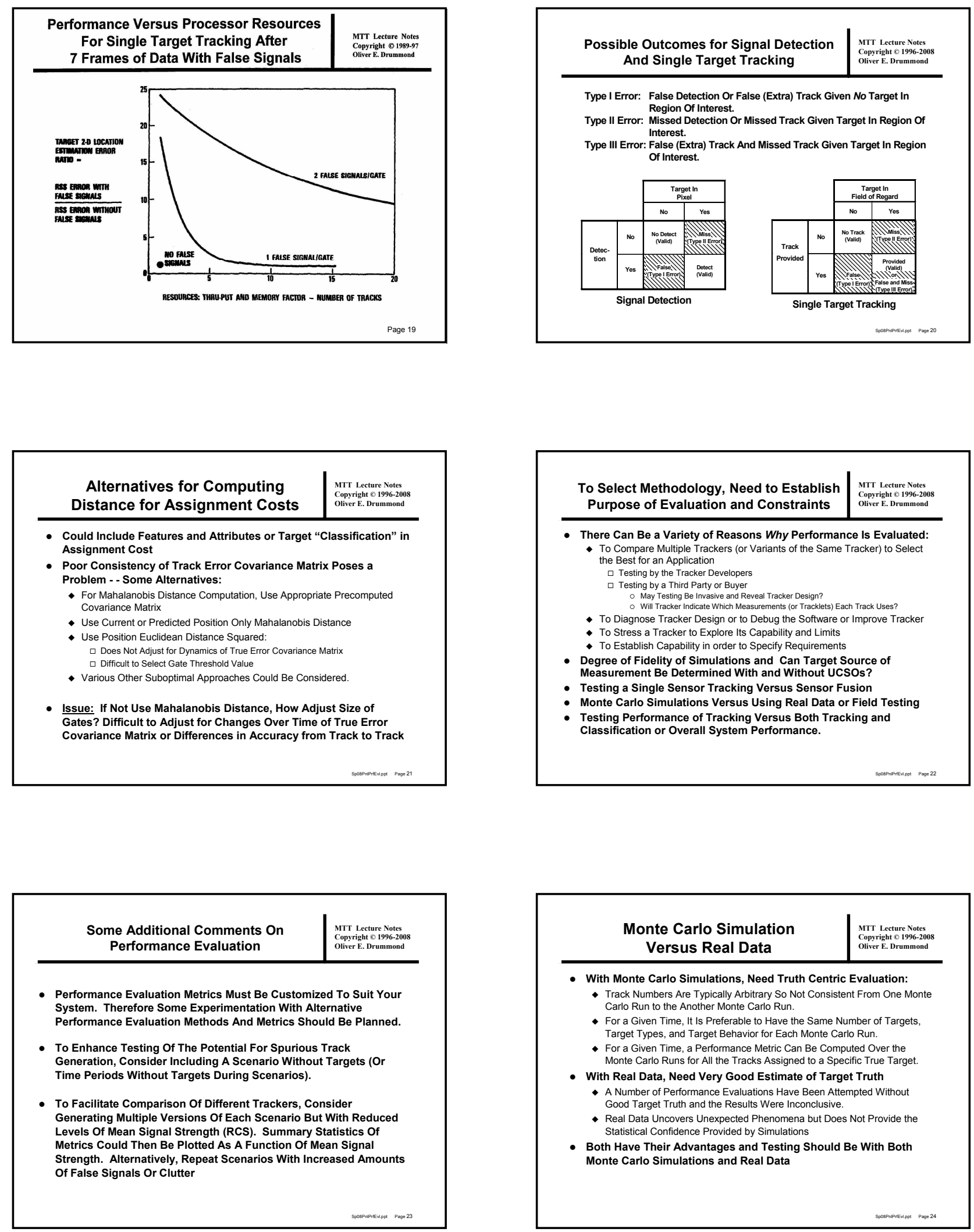

$\mathrm{XX}$ 


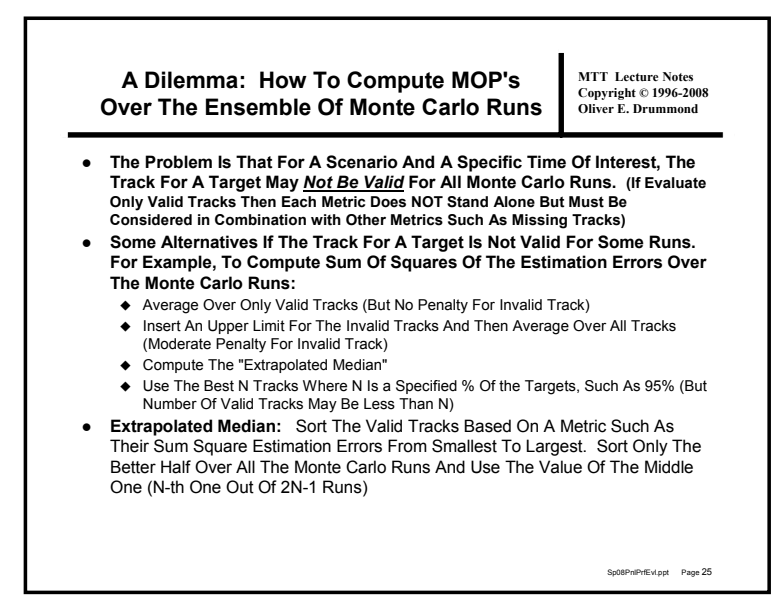

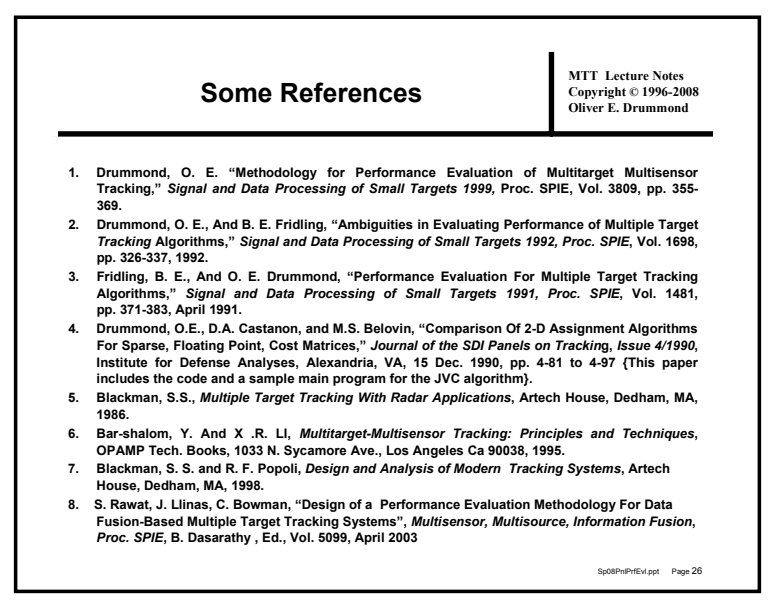




\title{
Target-to-Track Assignment in Performance Evaluation of Multiple Target Tracking
}

\author{
Oliver E. Drummond, Ph.D., P.E. \\ CyberRnD, Inc. and Consulting Engineer \\ 10705 Cranks Road, Culver City, CA 90230 \\ Internet E-Mail: Drummond@worldnet.att.net
}

Phone: (310) 838-5300

\begin{abstract}
Because there may be misassociations, performance evaluation of target tracking is complex due to ambiguities that can cause confusion about which target a track is following. This paper and companion PowerPoint slides presents a methodology to deal with these ambiguities so that performance metrics can be used to evaluate performance. The emphasis of this paper is on the first step of a two-step methodology, namely, the decision on which target to compare to a track so that measures of performance can be computed. However, no single methodology may be best for all the various types of tracking systems. In addition, no one methodology may be best for all the performance metrics that are appropriate to a specific system. Therefore, alternative target-to-track methods are described to permit selection of the appropriate methodologies for a specific tracking system.
\end{abstract}

Keywords: tracking performance evaluation, measures of performance, performance evaluation metrics, false tracks, error types, target tracking, multiple sensor tracking, multiple target tracking, sensor fusion, Bayesian method, Kalman filter, and target-to-track assignments.

\section{Presentation Summary}

Target tracking and classification problems can be broadly categorized into four generic types $[1]^{\otimes}$, as follows:

1. Sensor tracking of a single (bright) target

2. Tracking of large targets

3. Tracking of medium sized targets

4. Tracking of small targets.

Note that the size indicated in this list is in terms of the number of resolution elements or pixels of a target. Typically, a small target is less than 100 pixels. The algorithms used in the signal, image, and track processing for each of these types of problems differ substantially. Since each type of tracking problem poses different algorithm development issues, this paper and the accompanying Power Point presentation concentrate on only one type, namely, the performance evaluation of tracking of small targets using either single or multiple target tracking methods. Multiple target tracking is a relatively new field. The first book dedicated exclusively to multiple target tracking was published in 1986 [2] and a number of recent books are available, such as $[3,4]$.

Target tracking exhibits properties and unexpected results that are not common to most statistical estimation tasks. For example, tracking exhibits properties significantly different from signal processing; in addition to Type 1 and Type 2 errors, tracking exhibits Type 3 errors, that is a combination of both missed and false tracks [5] $\{3,19\}^{\otimes}$. One of the major causes of unexpected results is that tracking involves random variables from both continuous sample space and discrete sample space. Accordingly, using a high fidelity simulation is a vital step in algorithm development [1] $\{3\}$. In addition, tracking performance evaluation is both complex and somewhat controversial.

\footnotetext{
${ }^{\otimes}$ Note: $\{$ braces $\}$ refer to pages of the PowerPoint presentation and [brackets] refer to references in Section 2.
} 
Ultimately, performance of tracking algorithms is judged by the success of the system that they support. Tracking performance evaluation metrics serve as a vital intermediate measure of tracking effectiveness, to diagnose the algorithms, and to predict tracking performance for use in system studies and establishing requirements. However, ambiguities can occur in evaluating performance because of misassociations [6]. Misassociations can cause missed targets and false tracks such as redundant, spurious, switched, and lost tracks $\{4\}[5]$.

As a result of misassociations, it may not be clear which target a track is following, if any. Measures of performance cannot be meaningfully evaluated with the aid of a high-fidelity simulation plus field-tests (or hardware-in-the-loop testing) without first designating that target each track is following. A number of evaluation methodologies have been proposed to address this problem $\{17,18\}$ and some involve multiple frame assignment approaches $\{17\}$ [5]. Care is needed not to use a methodology that gives unfair advantage to one tracking approach over another. Both the target-to-track assignment and the evaluation metrics need to be customized to the sensor application of interest and the purpose of the performance evolution task $\{22,23,24\}$.

One relatively simple methodology for resolving these ambiguities is to use a 2-D assignment algorithm to uniquely assign the targets to tracks $[6,7]\{10,11\}$. The use of the statistical (Mahalanobis) distances between targets and tracks for the cost elements in the assignment matrix tends to treat the alternative tracking algorithms fairly. Then the tracking errors and other measures of performance can be computed given these unique track-target assignments. This is a two-step methodology $\{6,14\}[1]$.

In Step 1, the targets-track pairs are identified from the best unique assignment of targets to tracks. The assignment of targets to tracks could be based on the distance between each possible track-target pair and then minimize the sum of distances. If the tracker computed estimated state error covariance matrices are realistic, then the Mahalanobis distance is a meaningful statistical distance that tends to treat all applicable types of trackers fairly. An alternative is to compute the benefit of alternative track-measurement pairs based on the target source of each measurement of a track, which will be referred to simply as track purity $\{15\}$.

The use of track purity in target-to-track assignment requires two special computations $\{12,15\}$. First, it is necessary to determine how much each target contributed to the detection of each measurement. This computation is not practical in some high fidelity simulations that realistically simulate unresolved closely spaced objects. The second computation is to report how much each measurement contributes to each track. If a third party is evaluating performance, the tracker developer might not want to reveal this information. Knowing the target source of each measurement and how much each measurement contributed to each track permits computing a metric of the track purity of each track-target pair that can be used as a benefit value to maximize the sum of benefits with an assignment algorithm.

In Step 2, the performance evaluation metrics are computed and the results are recorded and plotted $\{14\}$. Some of the common measures of performance include the root mean sum square of the error biases, the position root mean sum square errors (rmse) and the velocity rmse; covariance consistency; the number of misassociations; track purity and duration; time to initiate tracks; and the number of missed, switched, and false tracks [8]. If the system involves multiple platforms, then performance metrics may also be needed to determine if all platforms exhibit the same information about the threat and friendly forces (SIAP). Typically for a tracking application, no single critical performance metric can be selected that can be used to evaluate the trackers. One reason a collection of metrics is needed is that usually the tracker parameters could be adjusted to favor one metric at the expense of others.

Both tracking performance and required hardware capacity should be evaluated since this is the major tradeoff in tracking. The choices of the algorithm architecture, algorithms, locations for each function, and the algorithm parameters will affect both performance and required processor capacity (plus communications load, if applicable). An example of this tradeoff between performance and required hardware resources is shown in $\{19\}[1]$. This figure summarizes results of the simulation of tracking a single target with data (that included false signals) from a single passive sensor. The results are shown after seven frames of data have been processed. The tracking algorithm was similar to a single-target version of so-called multiple-target, multiple-frame assignment. The number of frames in the sliding window was varied from 1 to 6 so that the curve in the figure was obtained. The values for the horizontal 
and vertical axes have been normalized by dividing by the corresponding value that is exhibited by tracking without false signals. Note that the results for the independent nearest neighbor (INN) tracking algorithm are at the left end of each of the two curves. This figure illustrates the major tradeoff between performance and the required processor capacity (processing time or required memory) for tracking with a single sensor.

\section{References}

1. Drummond, O. E., "Target Tracking," Encyclopedia of RF and Microwave Engineering, John Wiley \& Sons, Vol. 6, pp. 5081-5097, 2005.

2. Blackman, S. S., Multiple Target Tracking With Radar Applications, Denham, MA: Artech House, 1986.

3. Bar-Shalom, Y. and X. R. Li, Multitarget-Multisensor Tracking: Principles and Techniques, Los Angeles: OPAMP Tech. Books, 1995.

4. Blackman, S. S. and R. F. Popoli, Design and Analysis of Modern Tracking Systems, Norwood, MA: Artech House, 1999.

5. Drummond, O. E., Methodologies for Performance Evaluation of Multitarget Multisensor, Signal and Data Processing of Small Targets 1999, Proc. SPIE, 3809:355-369 (1999).

6. Drummond, O. E., and B. E. Fridling, Ambiguities in evaluating performance of multiple target tracking algorithms, Signal and Data Processing of Small Targets 1992, Proc. SPIE, 1096:326-337 (1992).

7. Drummond, O. E., Multiple-object Estimation, Ph.D. dissertation, Univ. of California at Los Angeles, Los Angeles, CA, 1975, Xerox Univ. Microfilms No. 75-26,954.

8. Rothrock, R. L. and O. E. Drummond, Performance Metrics for Multiple-Sensor, Multiple-Target Tracking, Signal and Data Processing of Small Targets 2000, Proc. SPIE, 4048: 521-531 (2000). 

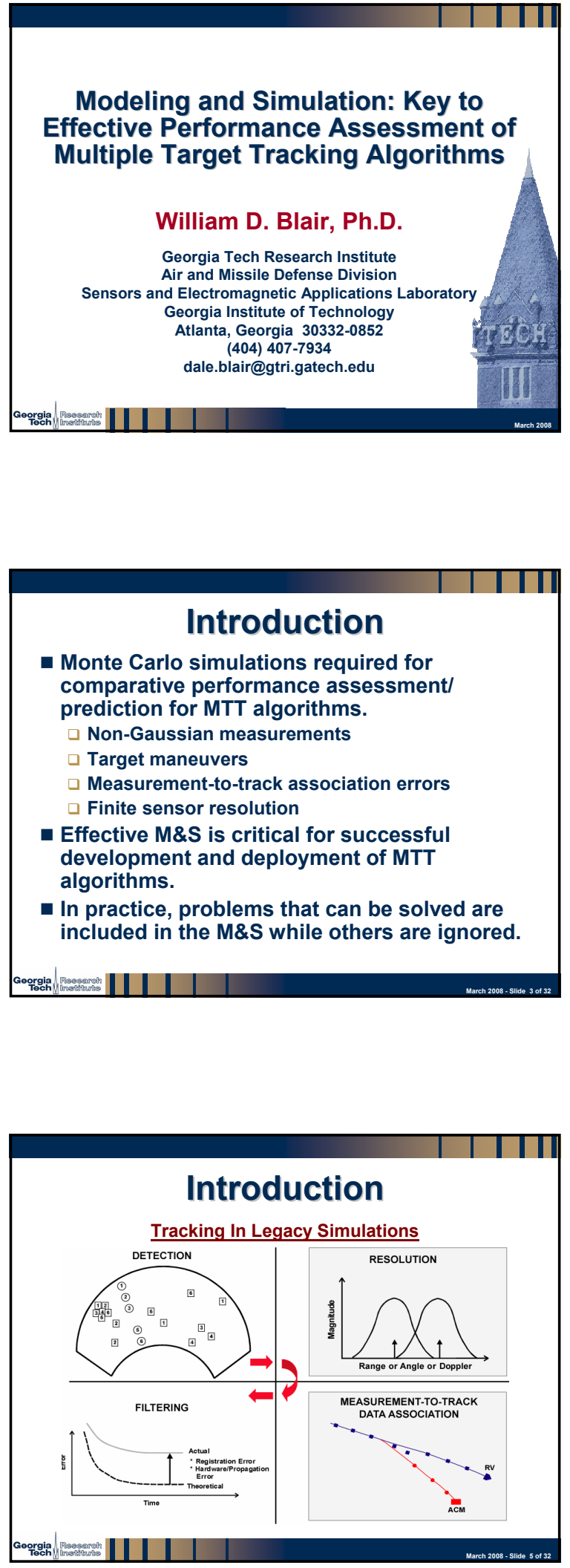
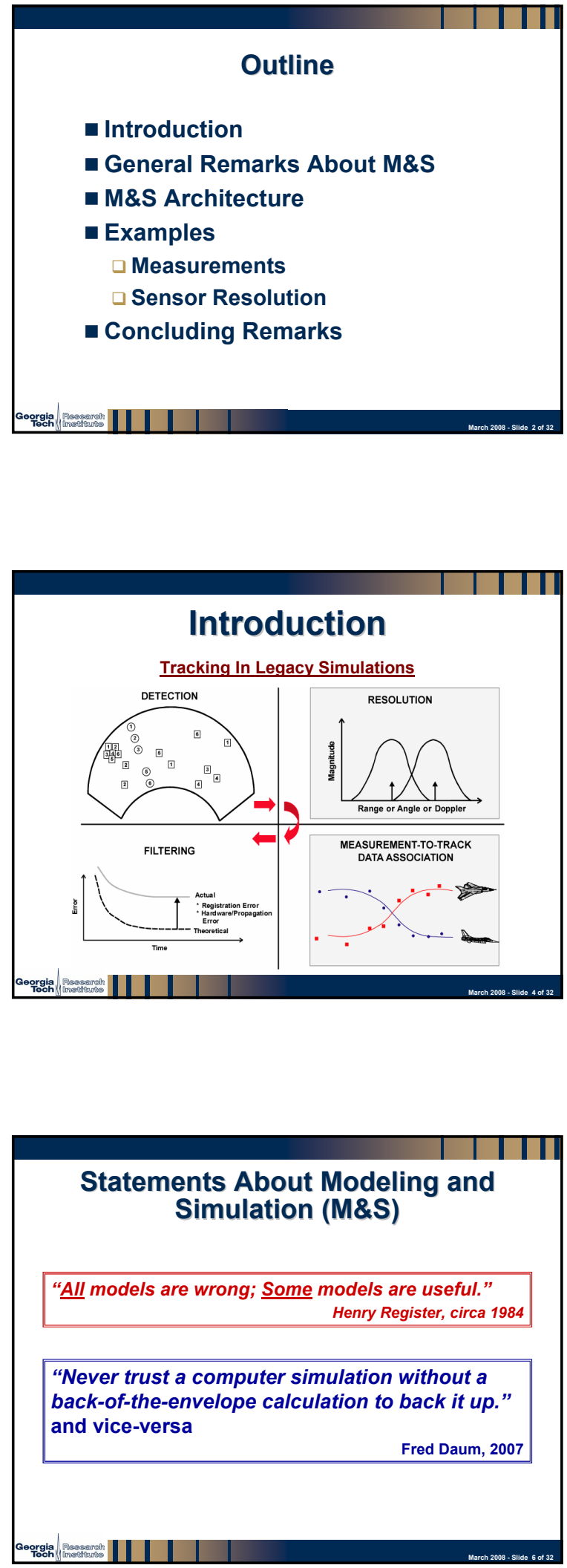

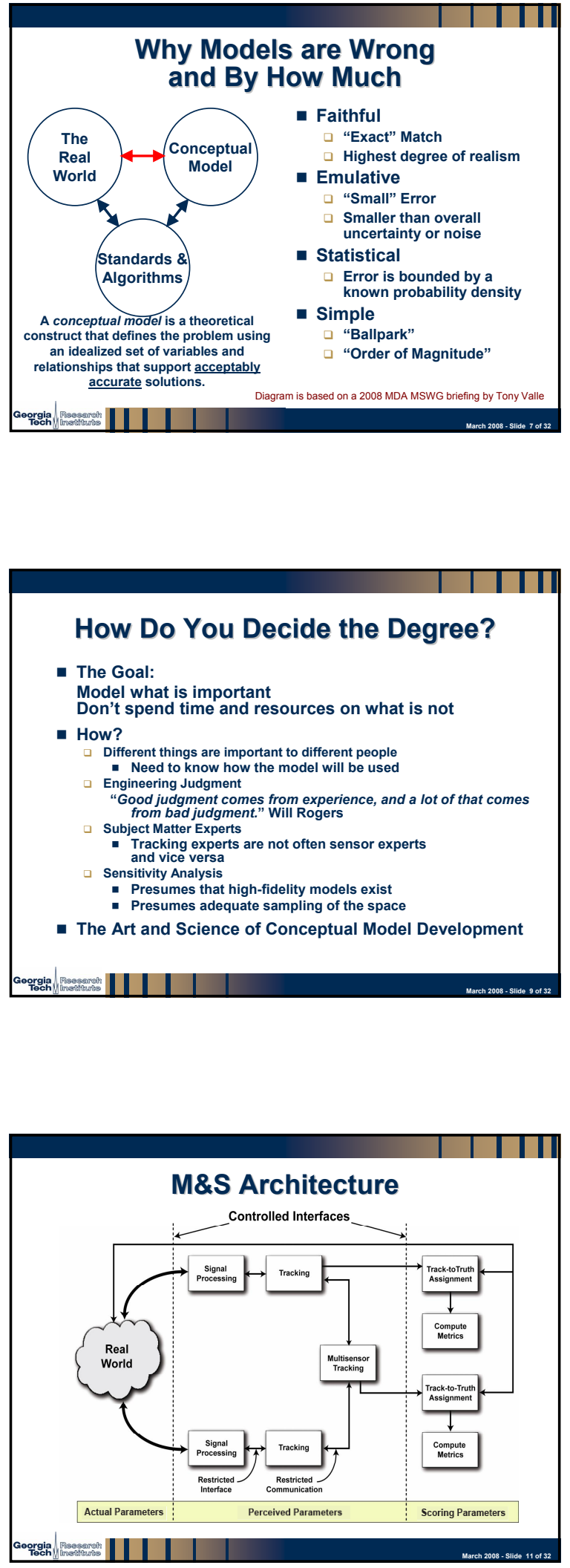
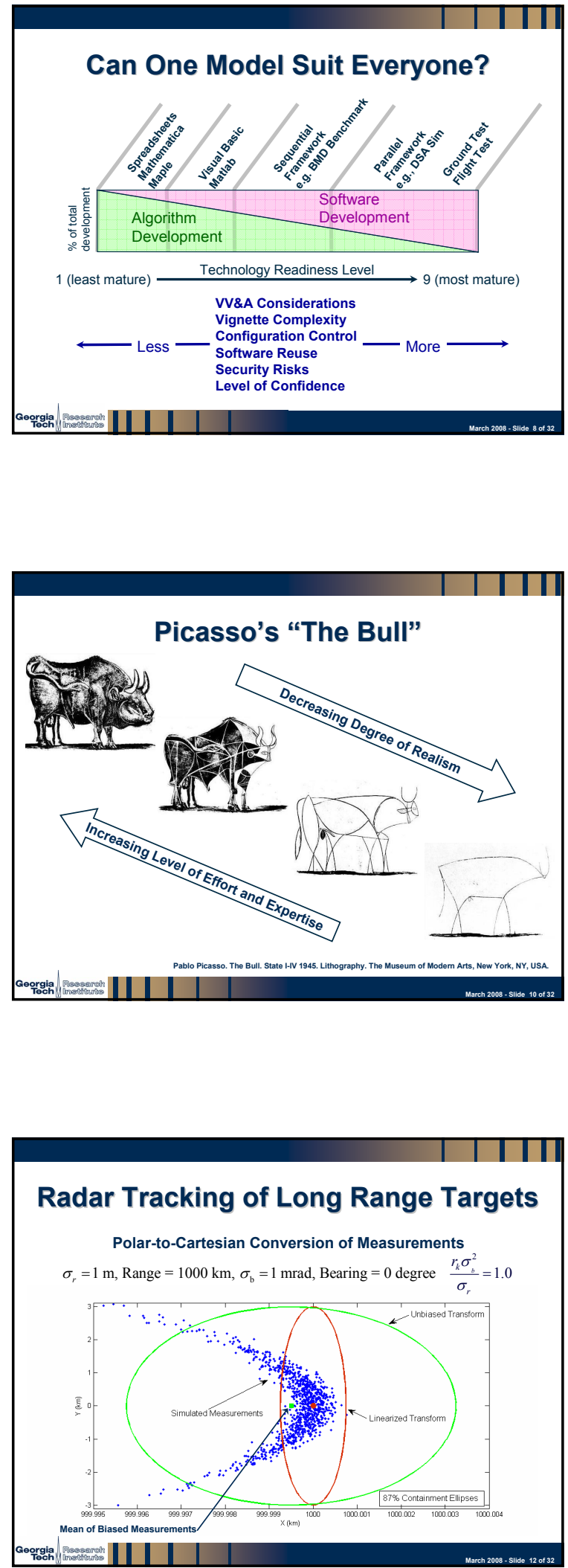

xxvi 

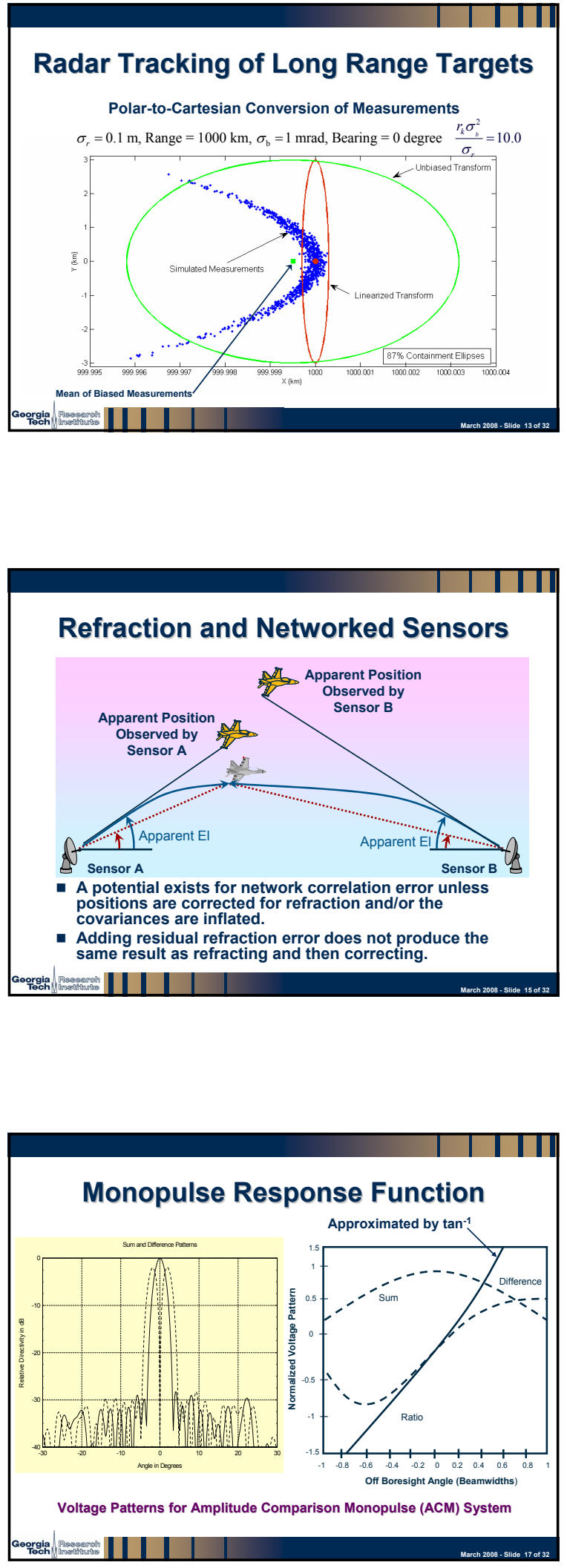
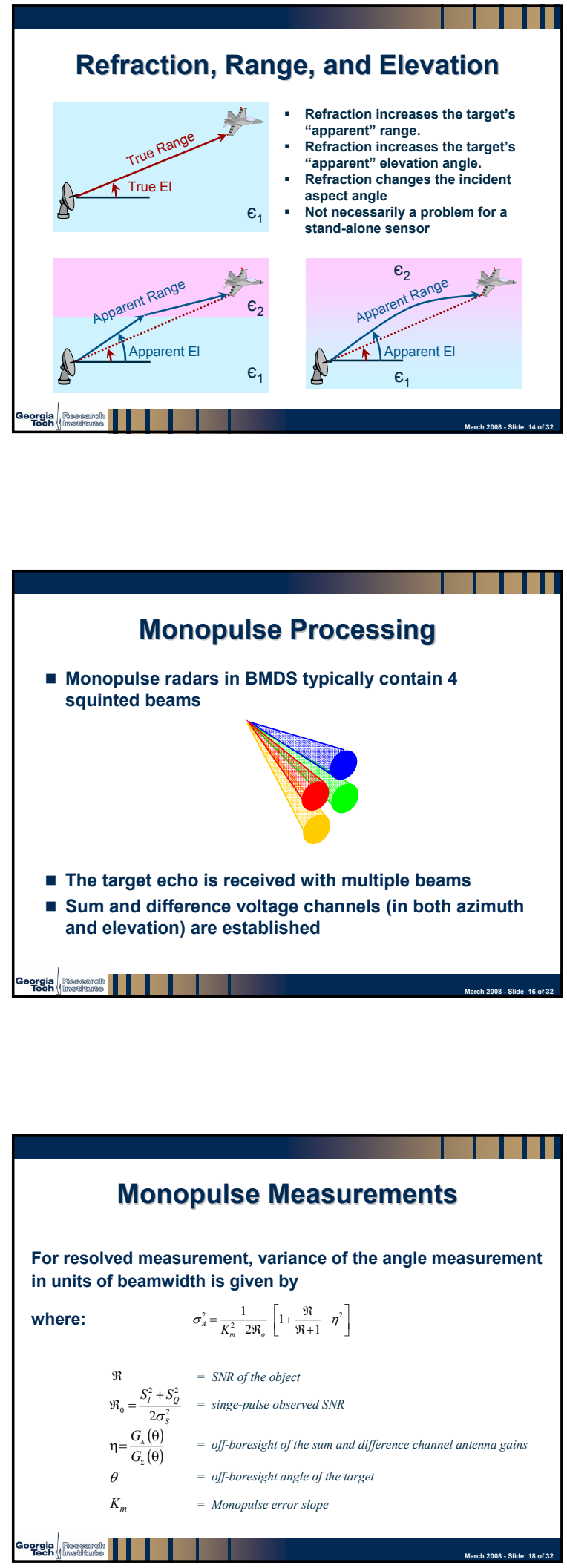

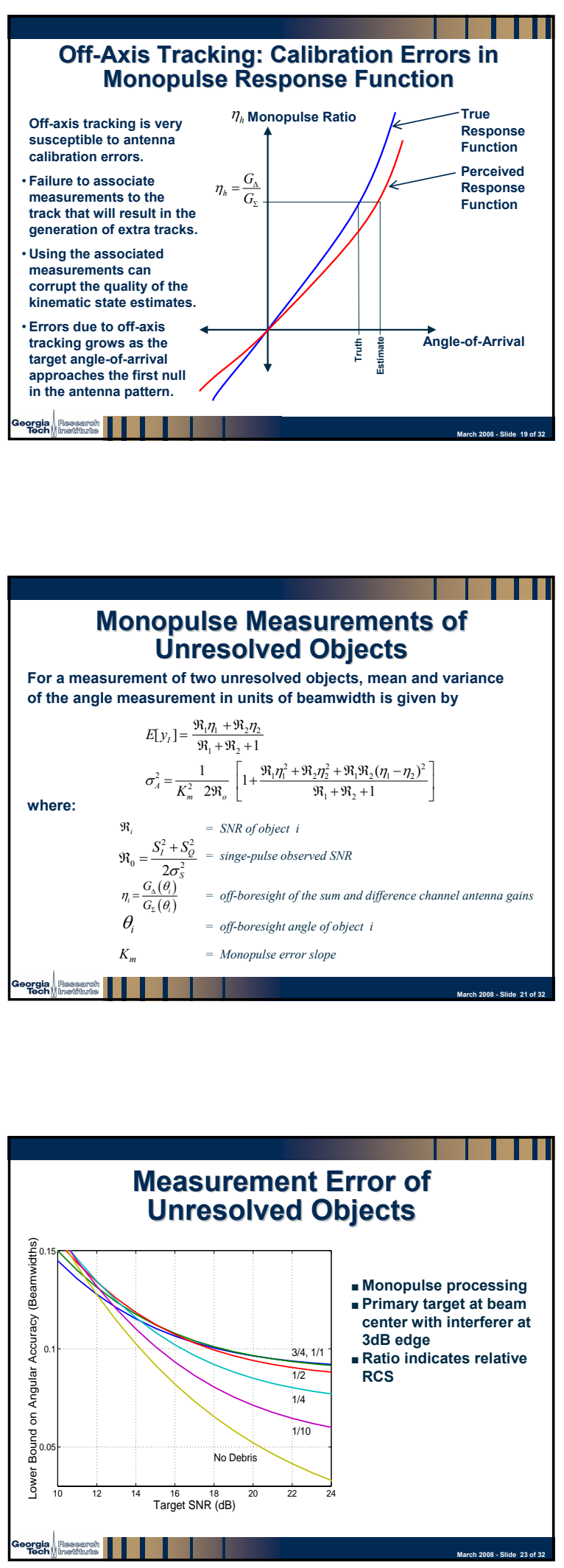
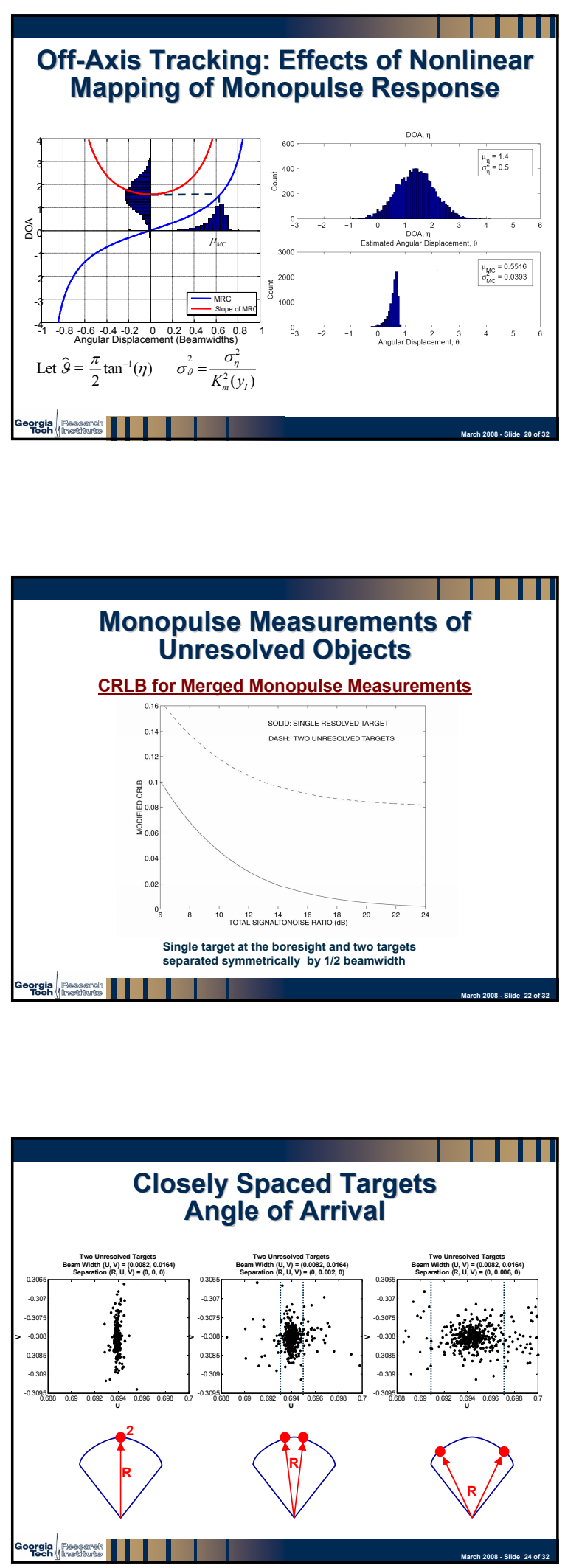

xxviii 

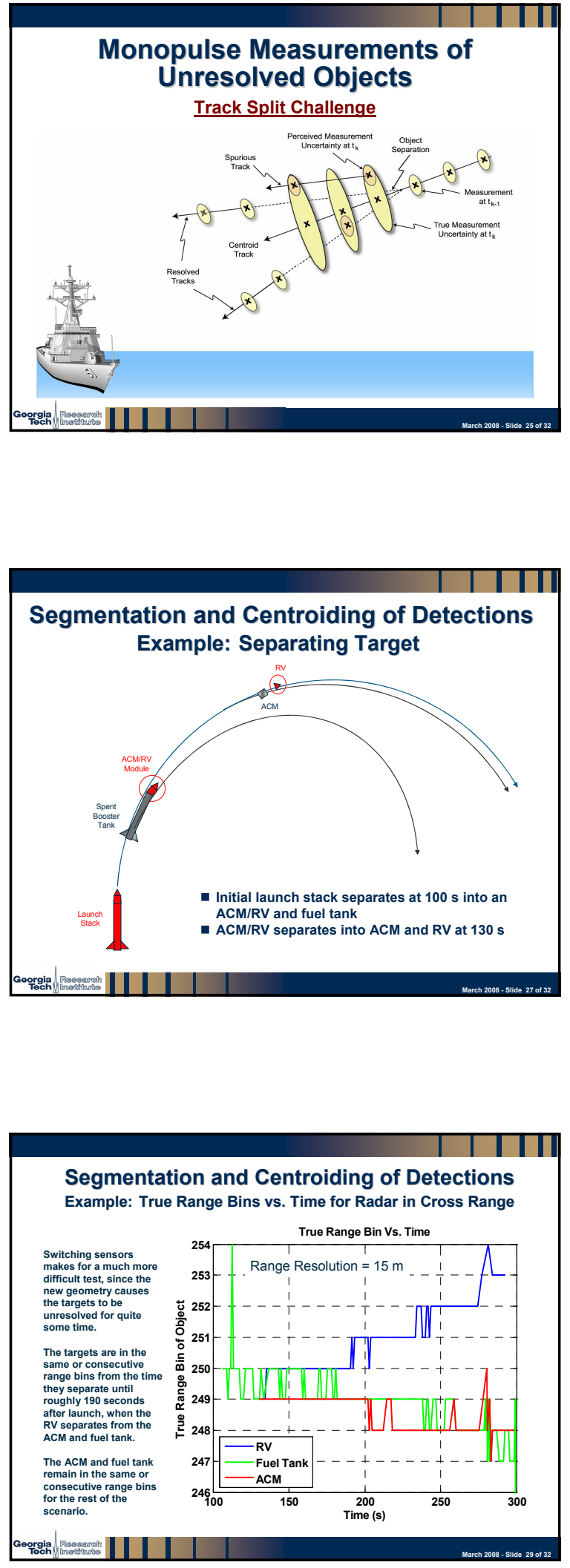
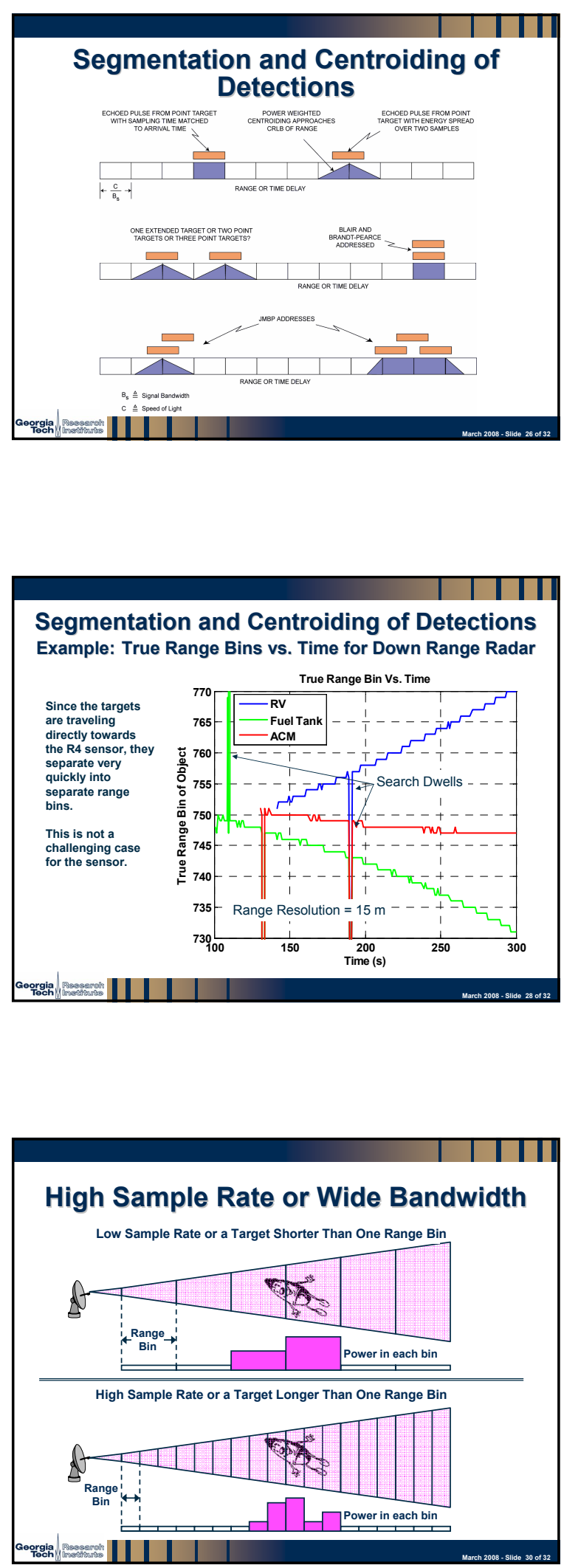


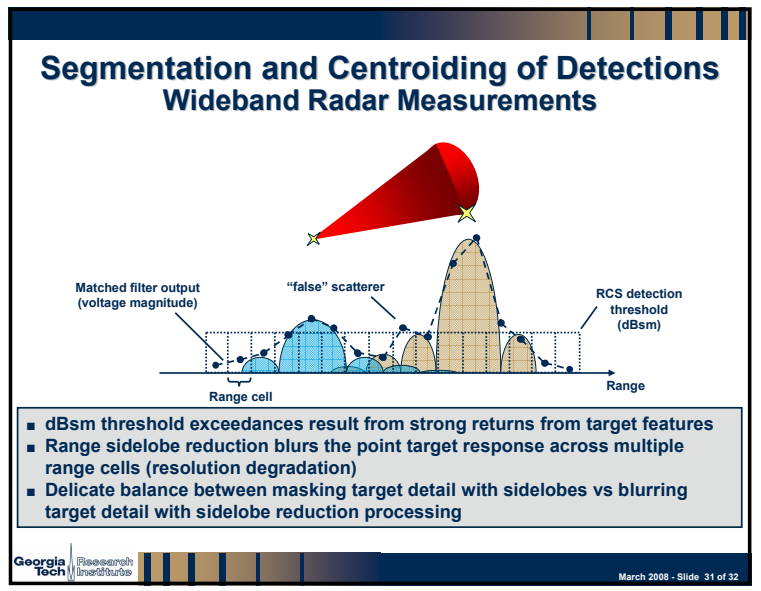

\section{Concluding Remarks}

- In practice, problems that can be solved are included in the M\&S while others are ignored.

- M\&S should be pursued so that the MTT algorithms will be successful when deployed.

- Effective M\&S is critical for comparative assessments of the performances of multiple algorithms

dBsm threshold exceedances result from strong returns from target features range cells (resolution degradation)

target detail with sidelobe reduction processin 


\title{
Modeling and simulation: key to effective performance assessment of multiple target tracking algorithms
}

\author{
W. D. Blair* \\ Georgia Institute of Technology, Atlanta, Georgia 30332-0857
}

\begin{abstract}
Since the errors sources associated with tracking maneuvering targets and measurement-to-track association for multiple targets are extremely complex, analytical calculation of performance are impossible for most all practical problems. Thus, one must resort to Monte Carlo simulations to assess the performance of the multiple target tracking algorithms. Therefore, effective modeling and simulation is critical to successful development and deployment of multiple target tracking algorithms. However, in many cases, the modeling and simulation is performed by the algorithm developer and it is effectively an "inverse" of the solution. Furthermore, many legacy simulations neglect the effects of sensor resolution and measurement-to-track association. Modeling and simulation for tracking algorithms should be decomposed into the "real-world," test article algorithms, and performance metrics. In this talk, the roles of each of these items will be discussed along with critical components of effective modeling and simulation and the challenges to be faced by the developer.
\end{abstract}

Keywords: ECM, multiplatform multiple target tracking, modeling and simulation

\section{INTRODUCTION}

Since the errors sources associated with tracking maneuvering targets and measurement-to-track association for multiple targets are extremely complex, analytical calculation of performance are impossible for most all practical problems. Thus, one must resort to Monte Carlo simulations to assess the performance of the multiple target tracking algorithms. Therefore, effective modeling and simulation are critical to successful development and deployment of multiple target tracking algorithms. However, many legacy simulations neglect the effects of sensor resolution and measurement-totrack association. In many cases, the modeling and simulation is performed by the algorithm developer and it is effectively an "inverse" of the solution. Effective modeling and simulation requires an understanding of the problem and the impact of the environment and hardware on the tracking algorithms. Algorithm developers or engineers must view the modeling and simulation as part of the challenge to make the algorithms work effective when deployed in the field. Along these lines, modeling and simulation for tracking algorithms can be decomposed in the "real-world," test article algorithms, and performance metrics. The roles of each of these items will be discussed along with critical components of effective modeling and simulation.

As illustrated in Figure 1, multiple target tracking can be viewed as four functional areas: detect, resolve, associate, and filter. As represented by the upper left corner, detection is the first step in the tracking of a target. Detection of the true target echoes occurs in the presence of false alarms and clutter detections. A threshold is applied to the signal amplitude to reduce the false alarm rate. However, a higher detection threshold reduces the probability of detection of the target. Thus, the detection threshold sets the false alarm rate and probability of detection and sets the performance limits of the tracker. Clutter is often countered with Doppler processing for clutter rejection and/or clutter tracking. As represented by the upper right corner, the second step of multiple target tracking is resolving the signals of closely-spaced targets into separate/isolated measurements. In radar systems, closely-spaced targets are typically resolved either in range, angle, and/or range rate. As represented by the lower right corner, the third step of multiple target tracking is assigning of measurements to tracks. This involves assigning measurements to existing tracks, initiating new tracks, and deleting tracks that are perceived not represent a truth object. As represented by the lower left corner, the fourth step involves the track filtering for estimating the position, velocity, and possibly acceleration of each target. Often, the performance of the track filter is analyzed under ideal conditions and its performance does not meet expectations when tested on actual sensor data. In most legacy simulations for tracking, finite resolution and the measurement-to-track data association are omitted from the modeling.

*dale.blair@gtri.gatech.edu; phone 1404-407-7934 


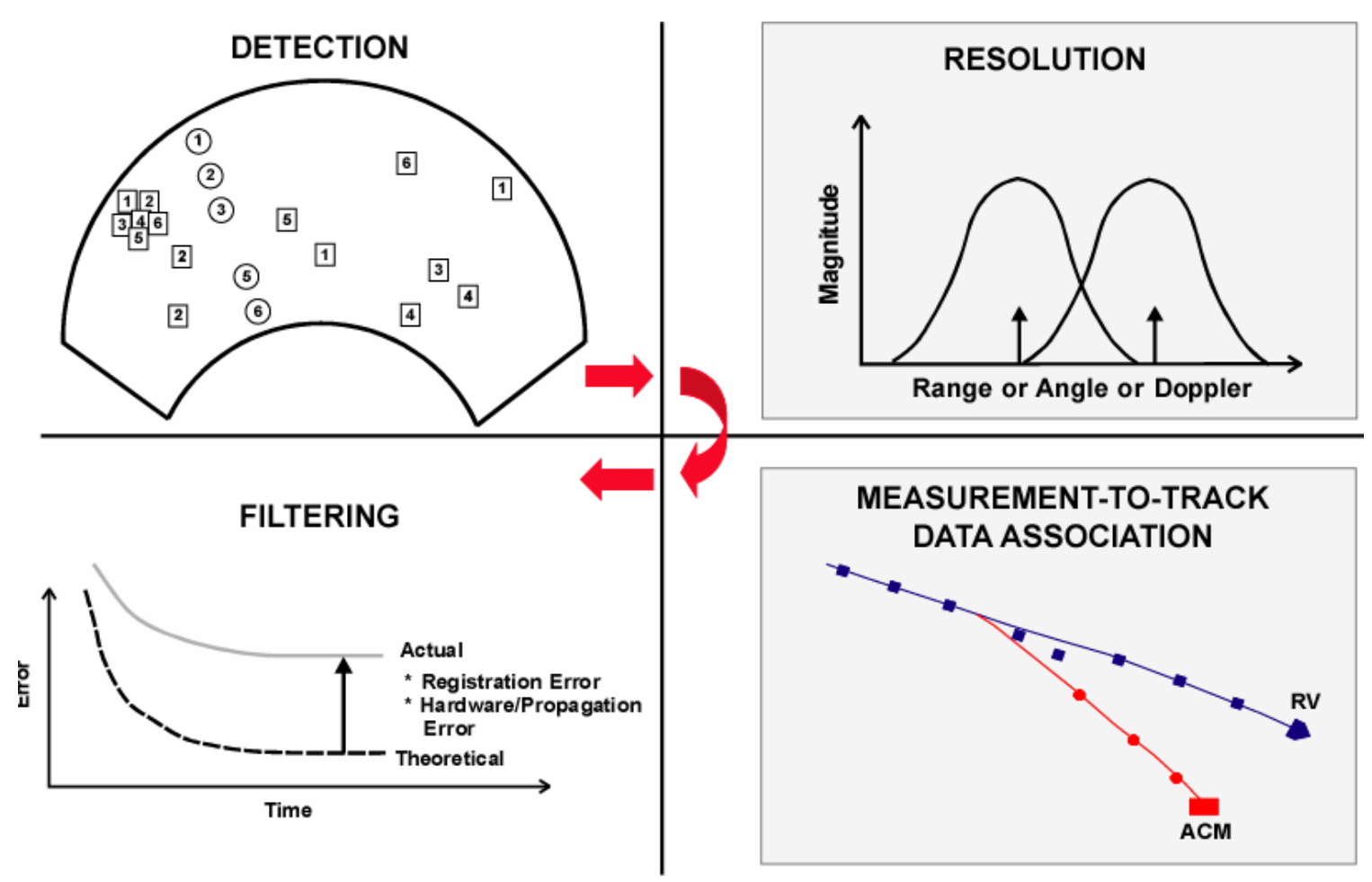

Figure 1. Functional Areas Associated with Target Tracking

\section{MODELING AND SIMULATION ARCHITECTURE}

The architecture of the modeling and simulation program is very important. Figure 2 is given to illustrate some of the critical issues. One of the most important features of a successful modeling and simulation program is a distinction between the "real-world" models and the signal processing and tracking algorithms. The models in the real-world should be separate and different from those included in the algorithms section. One should always remember that according to Henry Register "All models are wrong [1]" and be careful not to replicate the real-world models in the algorithms. A distinction must also be made between the actual parameters in the real-world model and the perceived parameters in the tracking algorithms. Information and the associated parameters that are not known to the tracking algorithms should be represented in the real-world and access to that information must be controlled by a restricted interface and any realworld information must be reconstructed or estimated from the data that is available through the restricted interface. The interface between the signal processing and the tracking should be restricted to that anticipated in the actual sensor system. The communications from the sensor trackers to the network-level trackers should also be restricted to reflect that anticipated in the actual network. A performance assessment should also be distinctive and separate from the realworld and the algorithms, and the interface to the between the real-world and the algorithms must be controlled. A common mistake that is made in this area is the scoring of trackers immediately after a measurement update of the track filters. Scoring trackers immediately after a measurement update gives one a false expectation of performance. The scoring times and other scoring parameters should be based on the system performance metrics and set separately from the tracking algorithms as in [2]. 


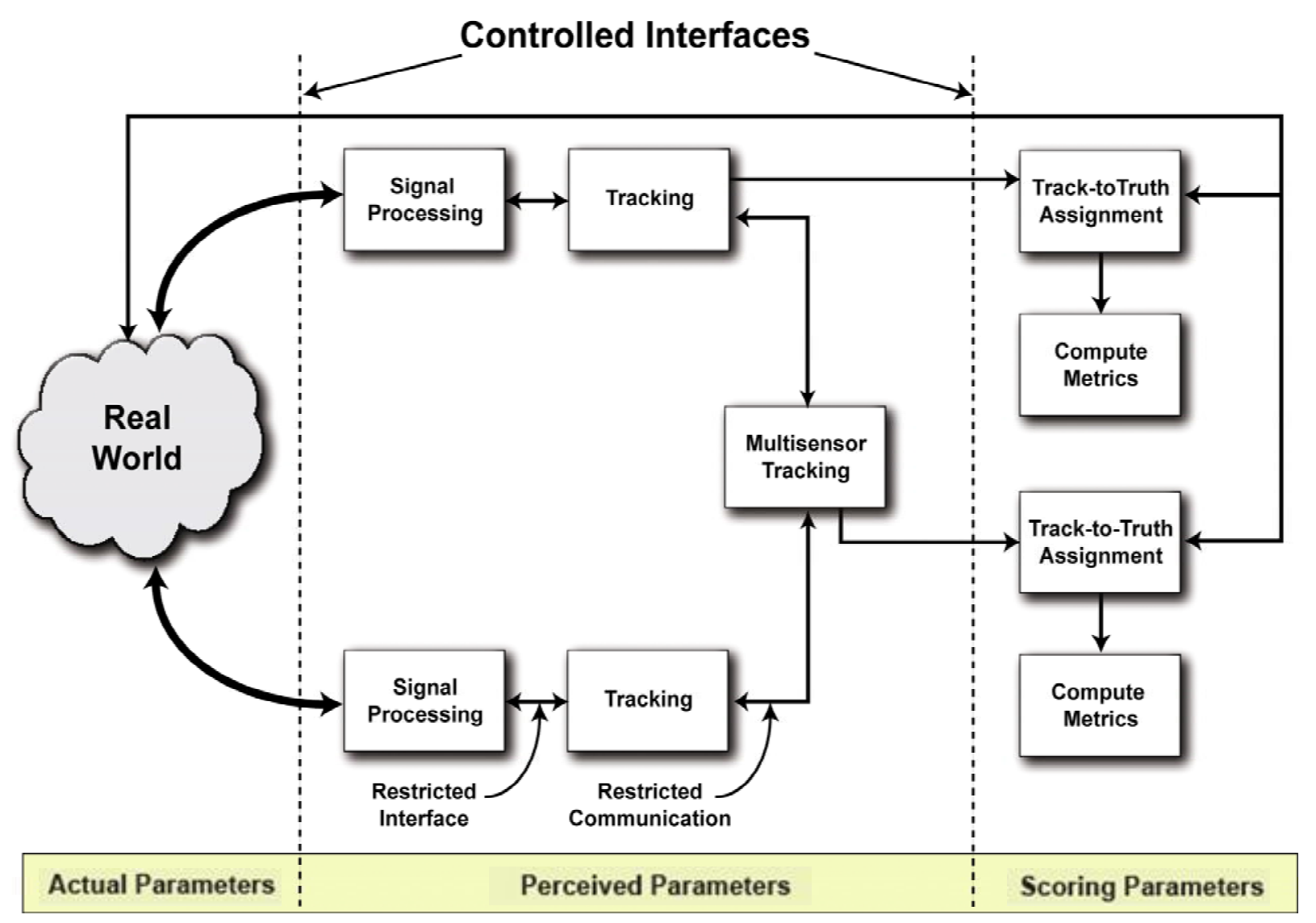

Figure 2. Modeling and Simulation Architecture for Multiplatform Multiple Target Tracking

\section{REFERENCES}

[1] Andy Register, Personal Communications, March 2008.

[2] Blair, W. D., Watson, G. A., Kirubarajan, T. and Bar-Shalom, Y., "Benchmark for Radar Resource Allocation and Tracking Targets In the Presence of ECM,” IEEE Trans. Aero. Elect. Sys., 1097-1114 (1998). 

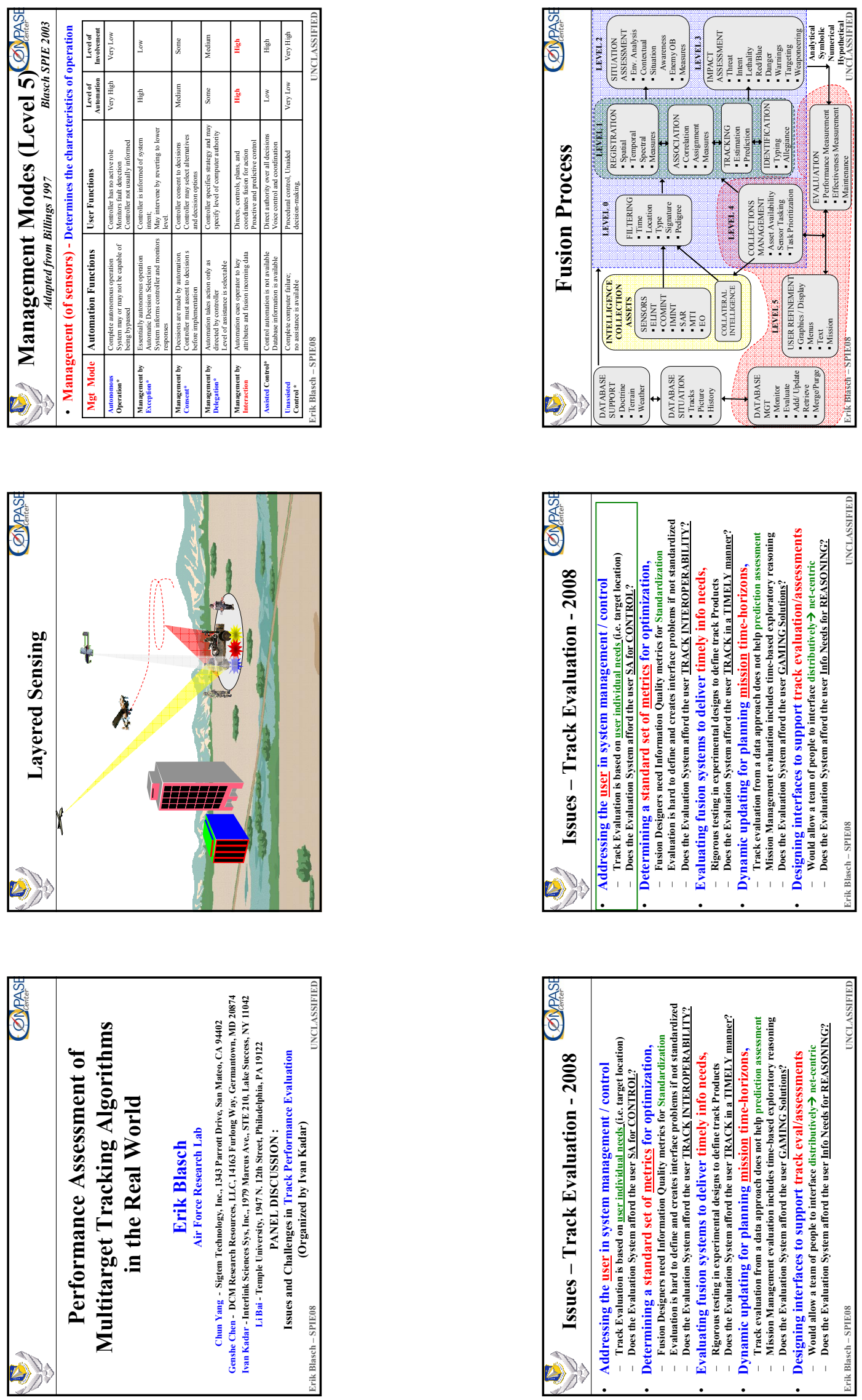

xxxiv 

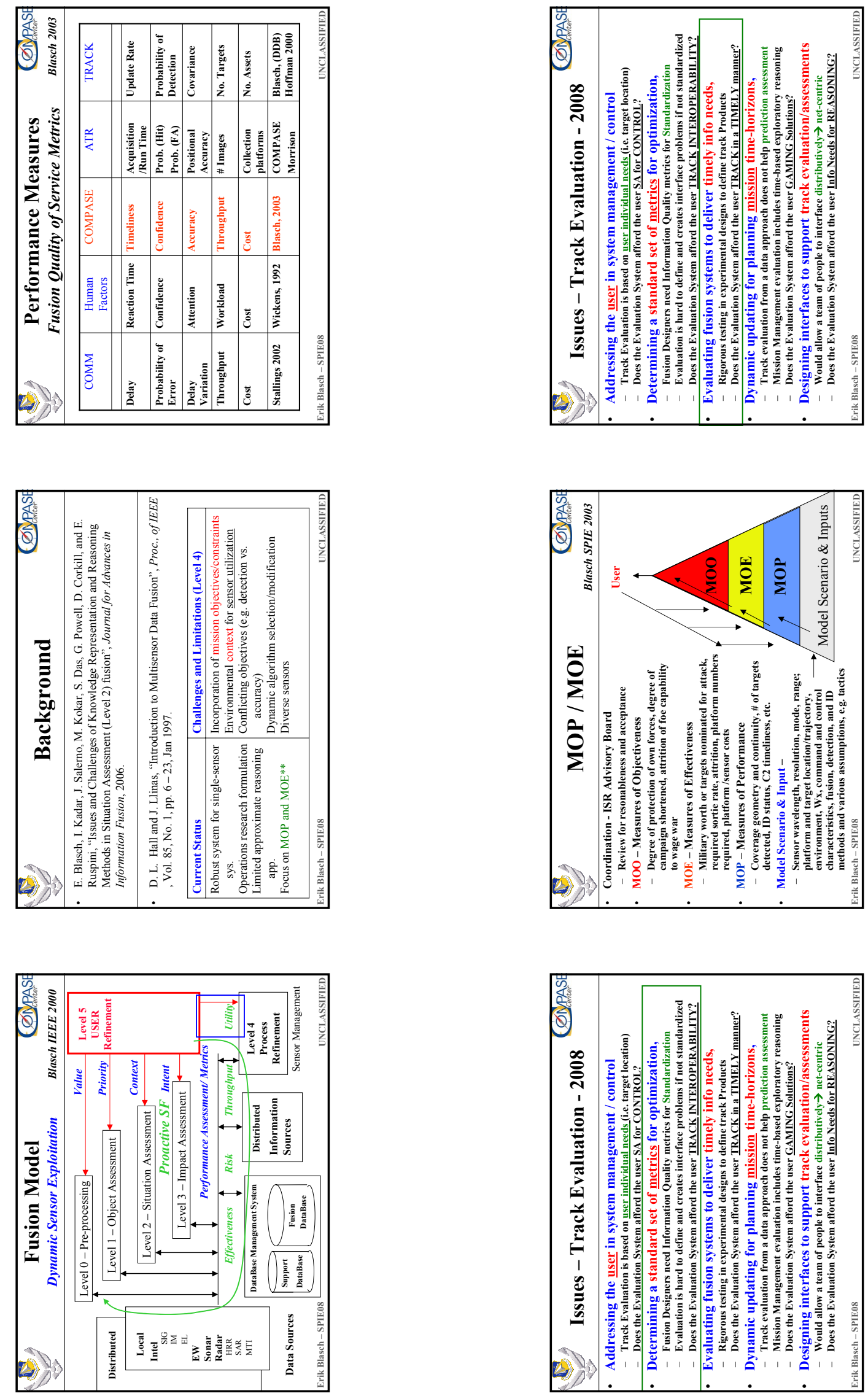

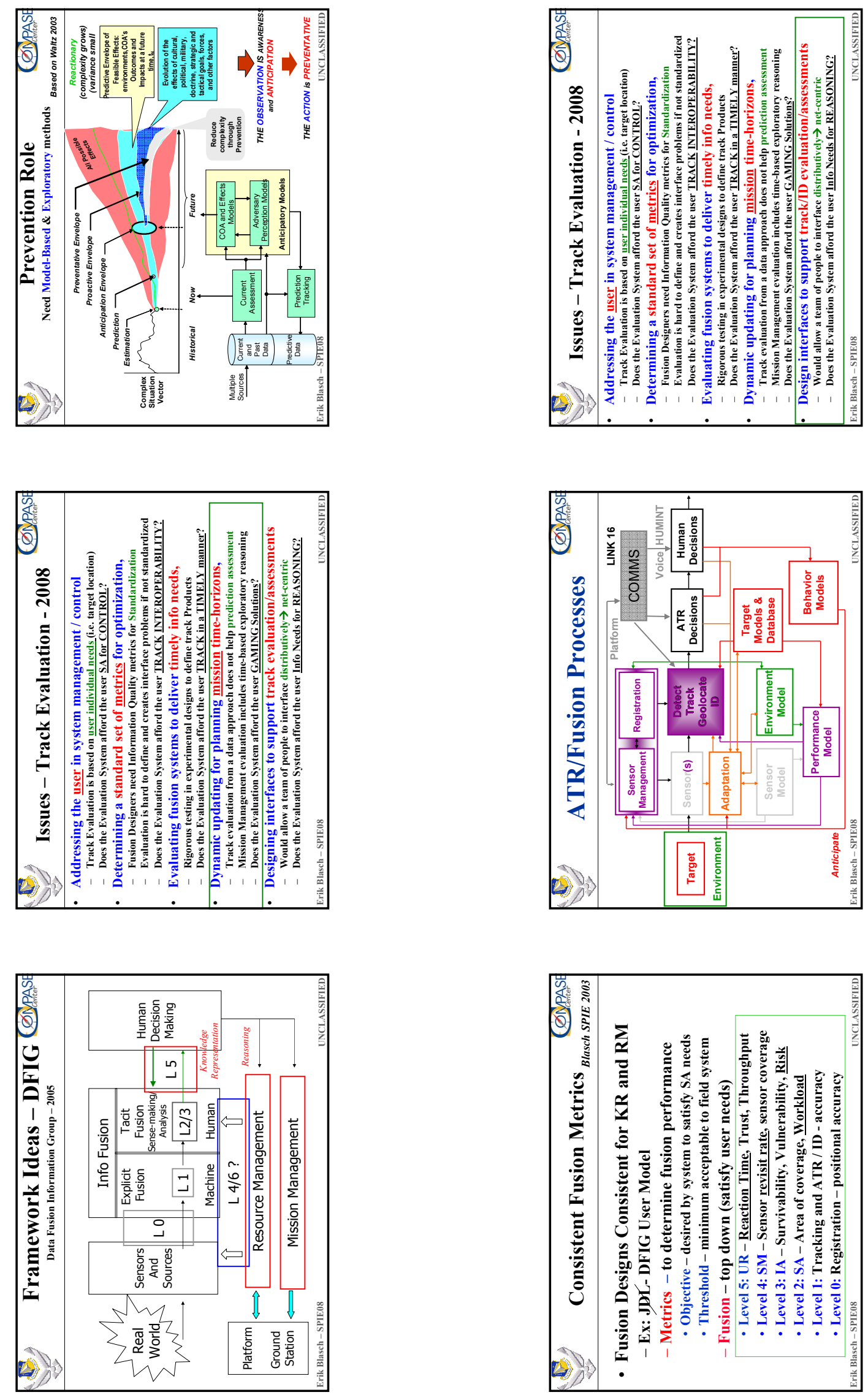

xxxvi 

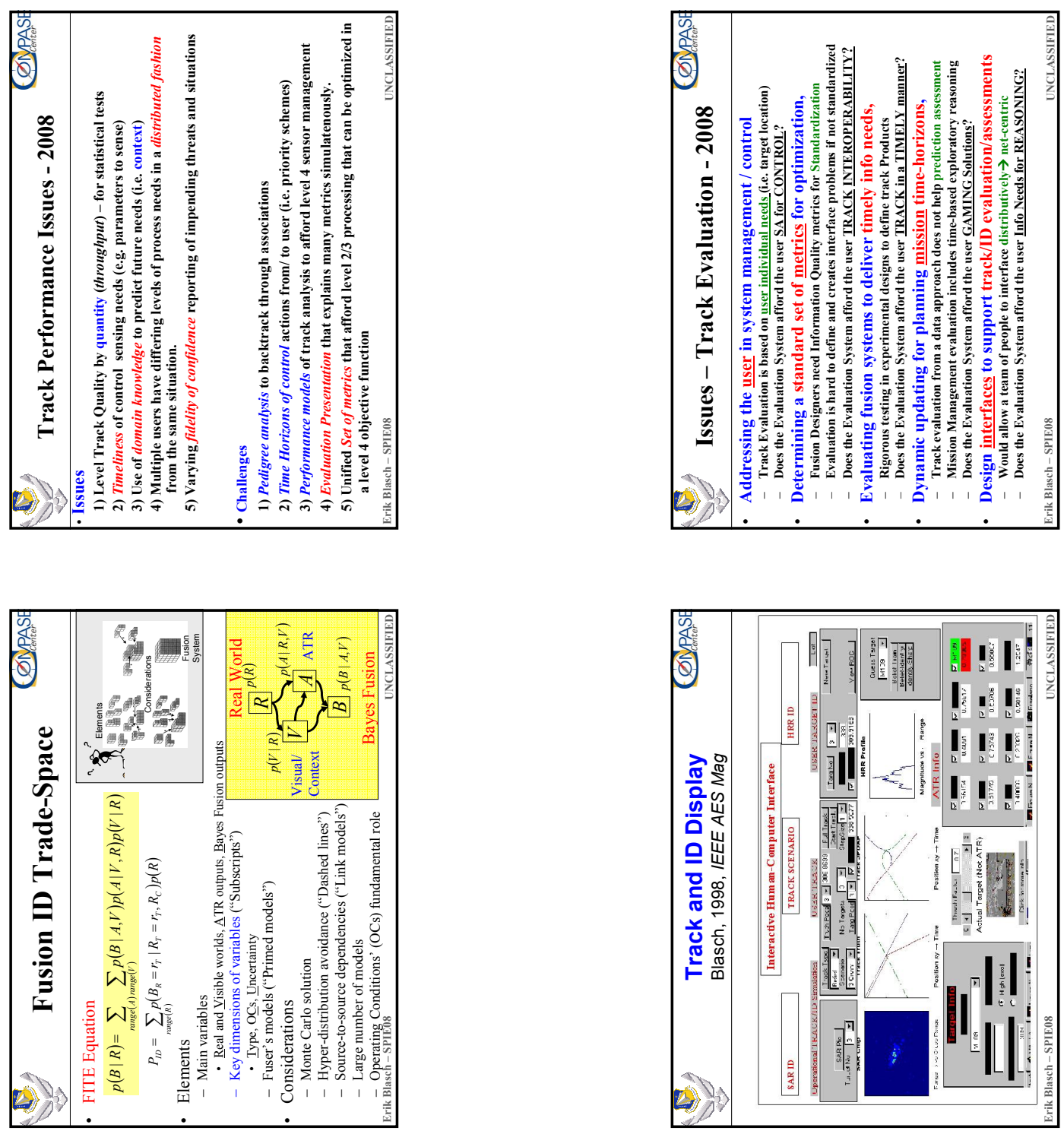

xxxvii 


\title{
Performance Assessment of Multitarget Tracking Algorithms in the Real World
}

\author{
Erik Blasch ${ }^{1}$, Chun Yang ${ }^{2}$, Genshe Chen ${ }^{3}$, Ivan $\operatorname{Kadar}^{4}$, and Li Bai ${ }^{5}$ \\ ${ }^{1}$ AFRL/RYAA - Fusion Evaluation, 2241 Avionics Cir, WPAFB, OH 45433-7001 \\ ${ }^{2}$ Sigtem Technology, Inc., 1343 Parrott Drive, San Mateo, CA 94402 \\ ${ }^{3}$ DCM Research Resources, LLC, 14163 Furlong Way, Germantown, MD 20874 \\ ${ }^{4}$ Interlink Sciences Systems, Inc., 1979 Marcus Ave., STE 210, Lake Success, NY 11042 \\ ${ }^{5}$ Temple University, 1947 N. $12^{\text {th }}$ Street, Philadelphia, PA 19122
}

\begin{abstract}
There are hosts of target tracking algorithm approaches, each valued with respect to the scenario operating conditions (e.g. sensors, targets, and environments). Due to the application complexity, no algorithm is general enough to be widely applicable, nor is a scenario narrow enough for a tailored algorithm. Thus, to meet real world goals, multitarget tracking (MTT) algorithms need to undergo performance assessment for (1) bounding performance over various operating conditions, (2) managing expectations and applicability for user acceptance, and (3) understanding the constraints and supporting information for reliable and robust performance. To meet these challenges, performance assessment should strive for three goals: (1) challenge problem scenarios with a rich variety of operating conditions, (2) a standard, but robust, set of metrics for evaluation, and (3) design of experiments for sensitivity analysis over parameter variation of models, uncertainties, and measurements.
\end{abstract}

Keywords: Tracking, Information Fusion, Performance Evaluation, Metrics, Scenarios

\section{INTRODUCTION}

Recent events have changed domain applications for multisensor information fusion and target tracking from locating a small number of large targets to maintaining tracks on a large number of targets of varying sizes. Increasingly complex, dynamically changing scenarios have evolved that require intelligent tracking strategies. These intelligent strategies have to be evaluated over various locations, observing changing targets, and detecting unknown threats. Such a scenario of interest for "Layered Sensing" is an urban setting for Homeland Defense. Layered sensing incorporates many research issues in intelligent tracking, sensor development, and information fusion. Figure 1 shows a layered sensing scenario that incorporates highaltitude platforms for target detection, surveillance unmanned aerial vehicles (UAVs) for target tracking, and individual audio reports from ground stations for target identification. To assist in the coordination of these layered assets, it is imperative to (1) understand the performance driving each system, (2) determine the

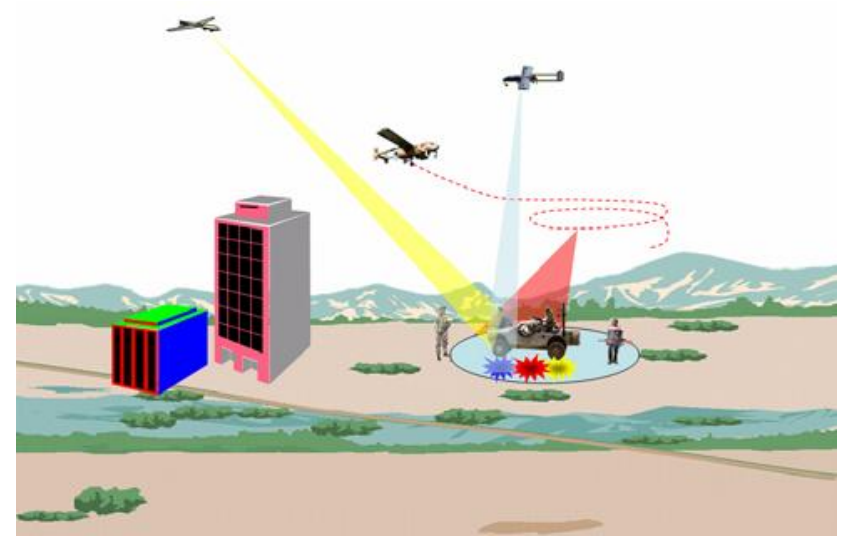

Figure 1. Layered Sensing. metrics for effective collaboration, and (3) proactively utilize the systems relative to the real-world scenario of interest.

Performance assessment of multitarget tracking (MTT) for the real world requires (1) pragmatic understanding of the algorithm designs, portability and applicability, (2) detailed testing over various scenarios and operating conditions, and (3) focused assessment of sensitivity analysis and performance metrics [1] through tailored design of experiments. While the 
MTT community has made progress in algorithm comparisons and metrics through the work of Blair in the Benchmark problems [2, 3], metrics and tailored target tracking performance evaluations from Drummond [4, 5, 6, 7, 8], and algorithms from Blackman [9] (as well as many others in the community e.g. [10, 11, 12]); there still is a need for reliable and designed testing scenarios to increase the understanding of the MTT approaches for the operational use of the techniques.

Real world applications of target tracking are embedded in larger operational goals such as surveillance, targeting, and force assessment that includes both entity estimation and situation control. The Information Fusion Community has adopted the Data Fusion Information Group (DFIG) model, shown in Figure 2, with emphasis placed on sensors and their estimations from the real world. Target tracking is but a piece of a larger information fusion system including user requirements, registration algorithms, and sensor management. Level 1 fusion, which includes target tracking and identification, is merely a

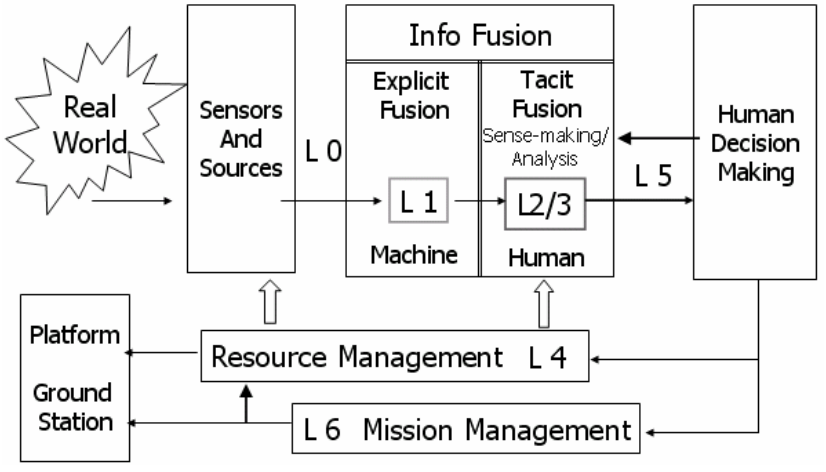

Figure 2. DFIG 2004 Model (Replacing the JDL Fusion Model) [13] technique to provide situational awareness. Situational awareness in and of itself is a picture of the environment that requires situation assessment (i.e. number of targets, movement of targets, and the identity of targets). This information aggregation supports a user's real world decision-making goals of security, strike packages, and positioning forces. However, the tracking performance assessment of the situation can also be viewed as a control problem. The control problem requires tools and techniques for decision support such as metrics and analysis to place sensors, waveform diversity to choose operating modes, and situation characterization to relay information to other distributed users. The real world is complex; however, effective methods of tracking performance analysis will aid users to appreciate and trust tracking systems, utilize the tracking results for situational awareness, and adaptively control sensors for effective performance.

This paper supports a plenary discussion on ideas to enhance research in MTT for real world instantiation of MTT algorithms. It is not meant to serve as a critic of the current state of the art, but rather to suggest ideas for future improvements and directions for the community to be able to (1) transition the techniques, (2) manage expectations for groups incorporating MTT into larger designs, and (3) develop the resources available for the next generation of tracking researchers.

Three areas of support that are important to the community are (1) scenarios [14], (2) metrics [15, 16, 17], and (3) design of experiments [18]. While these ideas are not novel, the packaged details in a "Challenge Problem" would support the community. As indicated, Dale Blair has supported a Benchmark challenge problem for many years [2], Oliver Drummond has supported a various host of metrics [19], and Jim Llinas and Center for Multisource Information Fusion [20] have done initial work on MTT design of experiments. Building on these developments, the MTT community can support future challenge problems so that continued understanding, testing, and focused assessment can improve MTT research developments.

The paper describes brief attributes where MTT performance assessment can be furthered for real world realization of the techniques. Section 2 starts with scenario designs in Challenge Problem development. Section 3 places emphasis on a robust set of metrics that needs to be adopted as a standard for the tracking community. Section 4 describes a design of experiments approach which typical complex systems designs utilize for understanding. Section 5 draws conclusions. 


\section{SCENARIOS}

Tracking algorithms for the real world require a pragmatic understanding of the complexities surrounding the application such as the number and types of targets, the sensors including their physical parameters and communication links, and the varying environmental conditions.[21] Utilizing static and dynamic environmental conditions can lead to host of challenges for MTT algorithms. Typically, with weak applicability to the real world, MTT algorithms focus on performance assessment over different target densities, ordered set of detection policies (e.g. Probability of Detection $>0.8$ ), and various forms of clutter (e.g. Gaussian and Poisson).

As example of challenging scenario designs, the DARPA Dynamical Tactical Targeting (DTT) program [22] tested a variety of scenarios and evaluated system performance. Figure 3 represents the design space of different scenarios available for a given MIDB (Mission Data Base), Nominated Area of Interest (NAI), and air task order (ATO) of mission objectives. After the selected scenario conditions for each run were determined, a simulator was used to instantiate the truth information, such as actual target behaviors and sensors measurements. The simulator used the mean values and variance bounds associated with selected factors to create a half-day scenario which afforded a longitudinal performance analysis based on ergodic assumptions.

While the various MTT Performance Assessment techniques have been

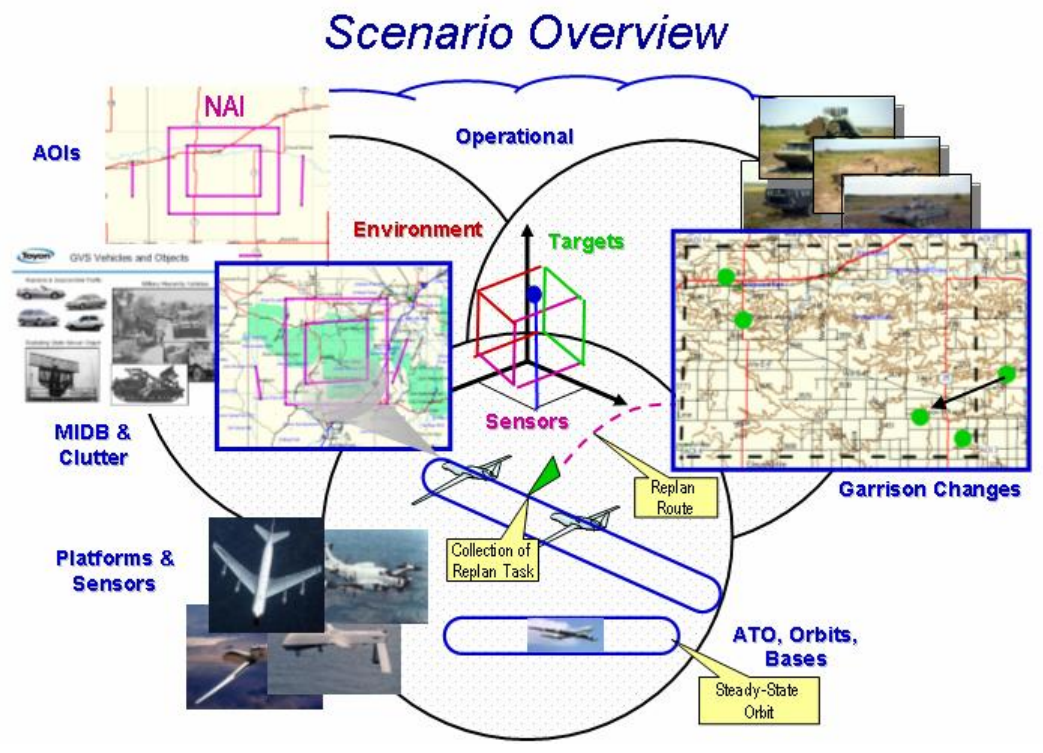

Figure 3: Scenario Parameters. [22] documented in the literature [23, 24], there is a long way ahead for reliable and robust performance assessment of real world systems. Issues of communications, measurement uncertainties, and models are important. As shown in Figure 4, there are many functions surrounding target tracking and identification. The first issue is to characterize the sensitivity of MTT systems to ancillary support data. With today's resources of GPS, terrain maps, and weather updates; it is important to utilize and capitalize on these products to support MTT assessment. It would be naïve to think that future MTT designs would not utilize this information. Likewise, the reliance on these technologies also becomes a sensitivity parameter of performance. For instance, research in terrain-aided navigation (akin to dead reckoning) is of interest to support navigational systems with a GPS dropout. Thus, MTT should

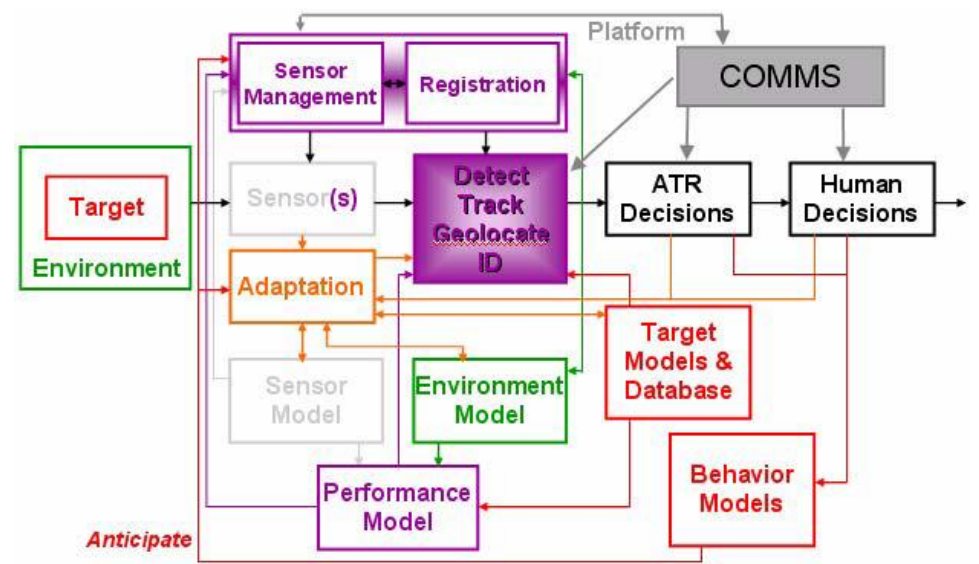

Figure 4: Support Needs for Target Tracking Performance explore scenarios over which reliable and unreliable support data is available. Additional ancillary information for real world testing includes data truthing, metric evaluation, and careful sensitivity analysis over operating conditions for model enhancements. 
The second aspect of scenario designs includes modeling. Modeling incorporates target kinematic and behavior models, sensor exploitation performance models, as well as the environment performance models (e.g. Digital Terrain and Evaluation (DTED) models). The tight restriction of MTT evaluation has typically included simple kinematic models for target behaviors. One way to account for the simplistic models is to use a Variable-Structure Interacting Multiple Model (VS-IMM) or Multiple Model Adaptive Estimation (MMAE) approach to fuse the models to cover varying target behaviors. Another issue of importance in the performance analysis is the track accuracy based on the smoothing of models or the track loss for the tailored use of a single model. Finally, the use of models is critical to sensor management which determines which sensors are positioned for measurements. If the sensor models are simple detection approaches, it does not afford a rich analysis of feature assessment for track maintenance. In summary, MTT performance assessment needs to refine the sensitivity of the results by way of determining the effects of models on MTT performance.

The third aspect of scenario design is related to MTT distributed information. These distributed aspects include the communications and user involvement. Information theory details the performance bounds relative to bandwidth and capacity. If future MTT systems are to be deployed for real world applications, there is a need to understand the track life and track maintenance of systems when there are sensor delays, data drop out, bit errors, and performance degradation factors. Similarly, real world tracking is not only based on surveillance, but user needs. The goal for MTT performance assessment is in "Performance Driven Sensing" when a true understanding of operator placement of the sensors for measurement becomes an integral part of the MTT algorithm. Rarely, is a high-value sensing asset allowed to operate autonomously. Furthermore, the more reliable and diverse systems are under management contention between commanders. Thus, a performance assessment should include distributed analysis of communication constraints and user control issues.

To utilize the various parameters in the real world analysis, the MTT community should support challenge problems and performance models to enhance the understanding of real world issues in MTT assessment.

\subsection{Performance Models}

A sensor model is derived from physical, simulated, or empirical analysis. The physical parameters can be incorporated into physical or analytical models such as the radar equation. A more detailed analysis of the sensor system feeding an MTT system would be an exploitation model, such as a SAR performance model [25]. There are a host of other sensors for MTT such as HSI or EO sensors [26, 27] that need development for models that are verified and validated. Second, there is a need for target models. In the case of MTT, many stationary target models exist such as the CAD models detailed in the DARPA MSTAR program.[28] Third, environment models such as DTED, Google Maps, and other data are available. Weather and terrain contour maps could aid in the understanding of target movement. The documentation and model fidelity [29] (for a given scenario) can be included in a benchmark challenge problem for MTT comparison and evaluation.

\subsection{Benchmark Challenge Problems}

Blair [2] created a benchmark challenge problem. The ATR Community has produced a set of challenge problems detailed by Arnold [30]. A scenario includes various kinematic target movements, possible sensor signals and target signatures, and terrain details. Ross et al. [31] and the MSTAR team created a set of operating conditions over sensor, targets, and environments.

As a quick summary, the following pieces constitute a "Challenge Problem:” [30]

- Problem Definition: The scope and significance

- Data: Applicable data for the defined problem

- Tools for reading and processing data

- Suggestions on training and test sets

- Characterization of the data

- Goals: Research challenge questions and suggested experiments

- Metrics: Guidance on reporting results

- Tools: Baseline code \& results: Shows reproducible minimum performance for the defined problem 
Scenarios provide data and support documentation for real world analysis either through analytical, simulated, or empirical results; however from software design "You can’t control what you can't measure” [32].

\section{MTT PERFORMANCE METRICS}

What you measure is what you get. To support performance assessment, there is a need for an authoritative standard, defined calculations, and suggested sensitivity metrics from which to test and evaluate algorithms. For example, military MTT is based on a host of metrics that start with the measurement (detection and cueing) and end with (engagement and assessment) as shown in Figure 5. MTT metrics can be viewed as a producer of or consumer of the information. If the user is only interested in target identity, then MTT kinematic behavior assessment would provide additional information for a goal outside traditional MTT processes. Likewise, MTT consumes the uncertainty and detection metrics from the sensors. Together the MTT system uses and consumes the metric information for analysis in conjunction with a sensor, algorithm, and platform manager.

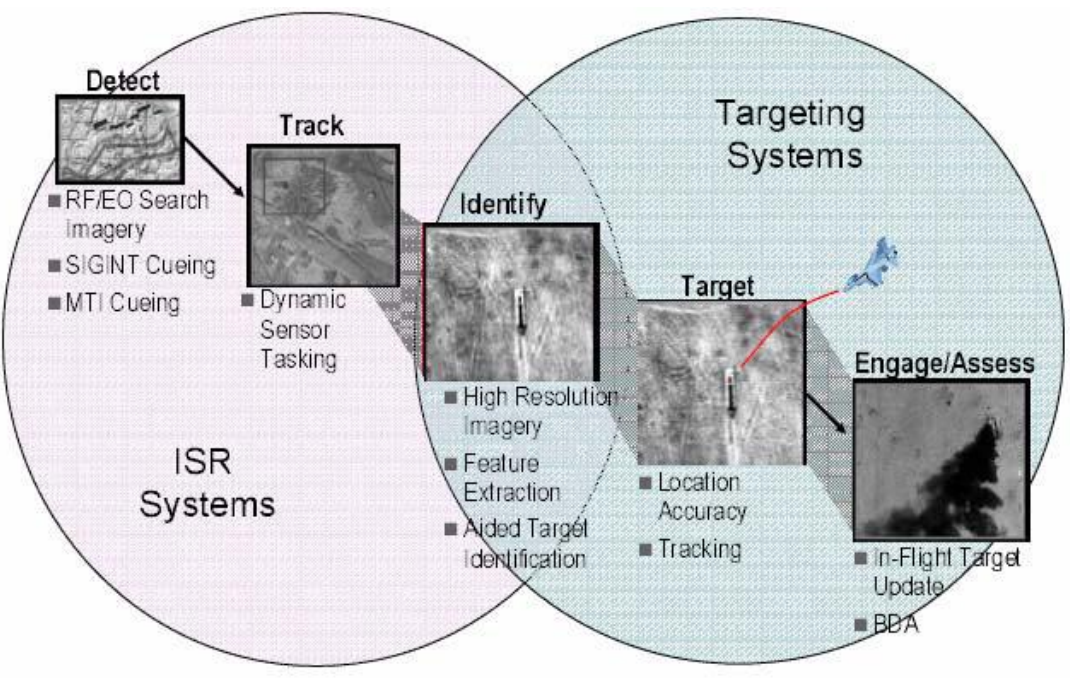

Figure 5: Kill Chain [33]

A host of MTT metrics have been described by Drummond, Blair, and Li. In each case, the metrics are associated with the algorithms themselves and provide a good taxonomy of issues to consider. In future real-world applications, there is a need to relate the metrics to user concerns above and beyond the standard metric of RMS position error for target location. One example is the communications community with a standard set of Quality of Service (QoS) metrics.[34] From the list of metrics, and important metric not currently considered for future real-world MTT analysis, includes Timeliness Variation. Sensor and information fusion systems require that the sensed information be communicated to the tracker in a timely manner with the associated throughput restrictions for hardware limitations. Communication methods transfer data from the sensor to the MTT center through routers, which can be modeled as a queue. For example, wireless systems will require ad hoc configurations of routers to transfer the information directly from the sensors to a mesh network. The mesh network, consisting of these routers, would then enable rapid transfer of the information to a distributed set of MTT sensors. Thus, to afford proactive MTT strategies, we need to consider the communication delays for timely decision making. For future MTT systems the Quality of Information will be important [35] as a sensitivity parameter as well as achieving the high fusion data quality (FDQ) [36] which may not be always commensurate with high QoS.

\subsection{Standardizing the MTT Metrics to Include System Metrics}

To design a complete MTT system, we need to address user information needs in the design and development process. Blasch [37, 38] explored the concepts of situation assessment (SA) by detailing the user needs of attention, workload, and trust which can be mapped into metrics of timeliness, throughput, confidence, utilization, and accuracy. Workload and attention can be reduced if the MTT system cues the operator to a few selected target descriptions (hence workload and timeliness). If the MTT performs better against a known a priori data set, accuracy and confidence increase, enhancing operator trust.

Dynamic situation analysis has three components: (1) dynamical responsiveness to changing conditions, (2) situational awareness, and (3) continual analysis to meet throughput and latency performance requirements. The combination of these three entities is instantiated by a tracking and ID [11, 12] system, an interactive display to allow the user to make decisions, 
and metrics for replanning and sensor management [39]. To afford interactions between future MTT system designs and users information needs, metrics are required. The MTT metrics should include timeliness, accuracy, throughput, confidence, and cost. These metrics are similar to the standard quality of service (QOS) metrics in communication networking [36, 40] and theory and human factors literature, as shown in Table 1 [1].

Table 1: Metrics for Various Disciplines [1]

\begin{tabular}{|l|l|c|l|l|}
\hline \multicolumn{1}{|c|}{ COMM } & Human Factors & Info Fusion & \multicolumn{1}{c|}{ ATR/ID } & \multicolumn{1}{c|}{ TRACK } \\
\hline Delay & Reaction Time & Timeliness & Acquisition /Run Time & Update Rate \\
\hline \hline Probability of Error & Confidence & Confidence & Prob. (Hit), Prob. (FA) & Prob. of Detection \\
\hline Delay Variation & Attention & Accuracy & Positional Accuracy & Covariance \\
\hline Throughput & Workload & Throughput & No. Images & No. Targets \\
\hline \hline Cost & Cost & Cost & Collection Platforms & No. Assets \\
\hline
\end{tabular}

The detailed metrics are required for a comparison analysis. For example, it is widely known that the interacting-multiple model (IMM) is best for maneuvering targets. In order to convey the results of tracking algorithm approaches, care must be taken to ensure that the performance analysis of the algorithm versus the data quality. For example, X Rong Li [41] has proposed relative metrics for analysis of trackers to separate the tracker performance from the quality of the measurements. Note: An IEEE Aerospace and Electronics Systems (AES) Standard Committee led by Blair is working on track fusion and target tracking terminologies.

\subsection{System Performance Metrics}

The goal of any multisensor system intelligent MTT analysis is to have a track gain over improved lifetime, timeliness, accuracy, and throughput. The information fusion gain can be assessed as Measures of Effectiveness (MOE) or Measures of Performance (MOP). MOPs include the standard MTT parameters of number of targets tracked, throughput, time, and $P_{D}$. MOEs include force protection, situation analysis, and event occurrence.

MTT performance metrics includes number of targets tracked; however, the system performance metric is throughput. Throughput can be determined as the average rate, peak rate, and variability of the system to deliver information to the user. The average rate (events processed / time) is the average load that can be sustained by the sensor over an extended period of time. The peak rate tells the network what type of surge traffic must be coped with, either by dedicating data-rate capacity or by allocating sufficient buffer space to smooth out measurement surges. Variability measures the source burstiness and is an indication of the extent to which statistical multiplexing is needed for distributed tracking.

Timeliness is QoS metric for system performance; however, system Delay (or latency) is a system-level metric which can be measured with time to assessment or delay variation. Transfer delay measures the delay imposed by the network on data transferring from a source to a destination. Delay variation is an important parameter for real-time applications, in which the data displayed at the destination must appear at a smooth continuous rate matching the rate generated at the source. Some of these issues were discussed in [36, 40] as they effect distributed tracking and fusion systems performance

\subsection{Robustness}

The MTT community is familiar with root-mean square error (RMSE), track purity, and track life [15]. While each of these is based on the scenario, it lends itself to false understanding of the tracker itself. To look at the tracker, many times the covariance information is used as a measure of the relative performance of the track estimation [9]. The MTT community, already accustomed to a system metric of track accuracy and track lifetime, should be acknowledging a system metric of robustness. Robustness includes specifications of designs (e.g. robust design), trusted reliability (e.g. robust decision making and estimation e.g., [ $42,43,44,45,46]$, and insensitivity to parameter variation (e.g. robust control). The goal for the MTT community is to explore the sensitivity of performance over a variety of conditions to ensure that the tracker designs relate to robust performance [47, 48]. Essentially, a robust assessment, extending from confidence and reliability [49], would lead to performance bounds that give an envelope of performance to support a managed expectation to users of MTT performance. In summary, the choice of metrics, either detailed in the challenge problem or supported by the tracking community, needs to be standardized, consistently calculated, and allow for sensitivity studies. 


\section{DESIGN OF EXPERIMENTS}

DOE supports the valid and verifiable direct, indirect, and interactive effects between the various operating conditions over the changes in these scenarios for a sensitivity analysis. Since real-world MTT incorporates a host of interactive effectives in a complex environment, there is no such analytical model to cover all aspects of the research. While the analytical approach is not without merit, only parts of the problem can be modeled. With some models, simulation studies can lead to an understanding of the domain. The MTT community is rich with papers utilizing kinematic models with simulated data to test out a set of operating conditions. There are even studies that include analysis over an empirical data set. However, the issue of the "Real World" incorporates both the expected and the unexpected events in a scenario. Thus, the MTT community needs to realize that the tracker is a piece of a larger complex system. Acknowledging that MTT is in a complex system leads one to seek complex performance analysis for which a standard approach is Design of Experiments.

Design of Experiments (DOE) [50] is a methodical way to control the performance assessment by changing certain parameters and regressing over the outcome, The purpose of using formal DOEs is to achieve the highest level of statistical significance of the tests while requiring the minimum experimentation e.g., [51]. DOE includes a host of techniques used to support the validity and reliability of testing complex systems while systematically understanding the process. Measures of Merit (or Figures of Merit) such as accuracy, confidence, and timeliness can be studied by changing parameters. One example is shown below of a DOE for MTT [22].

To effectively evaluate system performance, three types of parameters are assessed: (1) constants (e.g. NAI); (2) Monte Carlo variables (e.g. sensor variation), and (3) factors that change (e.g. number of targets) [21]. Table 2 shows the DOE analysis for the target, sensor, and environment EOCs (experimental operation conditions [32]). Constant parameters were determined from either un-modelable effects, issues that did not affect the outcome, or real-world operating conditions. Constant target behavior includes emissions and on-road travel while variable target behavior includes move-stop-move cycles, paths, and speed. The sensors and platforms are a result of the mission scenario. The sensor variables included exploitation accuracy and sensor factors including an On/Off bias selection. The largest category of scenario OCs is from the environment which includes weather, nominate area of interest (NAI) selected by the user, facilities of target initiation/termination, terrain/road conditions, and 'no-fly zones'. For each scenario, the NAI was fixed and available terrain and weather data provided.

Table 2: DOE of Test Scenarios. [22]

\begin{tabular}{|c|c|c|c|}
\hline OC Category & Parameter & Flat Terrain & Mountainous \\
\hline \multirow[t]{3}{*}{ Targets } & Targets & $6,12,25$ & $1,2,5$ \\
\hline & Moving Confusers (Dens. / AOI) & Low(10), 50 , High (1000) & Low (0), Med (10), High (25) \\
\hline & Routes-Stop-Move & Variable & Variable \\
\hline \multirow[t]{2}{*}{ Sensors } & Initial Start Points & Variable & Variable \\
\hline & Bias & On/Off & On/Off \\
\hline Environment & 'No Fly' Zones & Variable Area Locations & Variable Area Locations \\
\hline
\end{tabular}

\section{TRANSITION TO THE REAL WORLD}

Together the scenarios, metrics, and DOE lead to focused challenge problem sets for a performance analysis and repeatable understanding. Layered sensing can mean various things depending on the operating conditions. However, there are common themes associated with layered sensing MTT, such as dense targets, roads and infrastructure, and known sensor measurements. Scenarios require many novel MTT applications that are not well researched such as evaluation metrics, proactive control of sensors, distribution of information to users, usefulness of pedigree information, and robust metrics. To determine successful intelligent MTT processing strategies, an evaluation assessment is needed to determine if developments have increased the capabilities of the MTT solutions. Figure 6 shows a process model [52] detailing the processes of innovation to design to operations. From the MTT analytical model studies, towards simulations with generated and empirical data, MTT systems are ready for real-world DOE testing. 


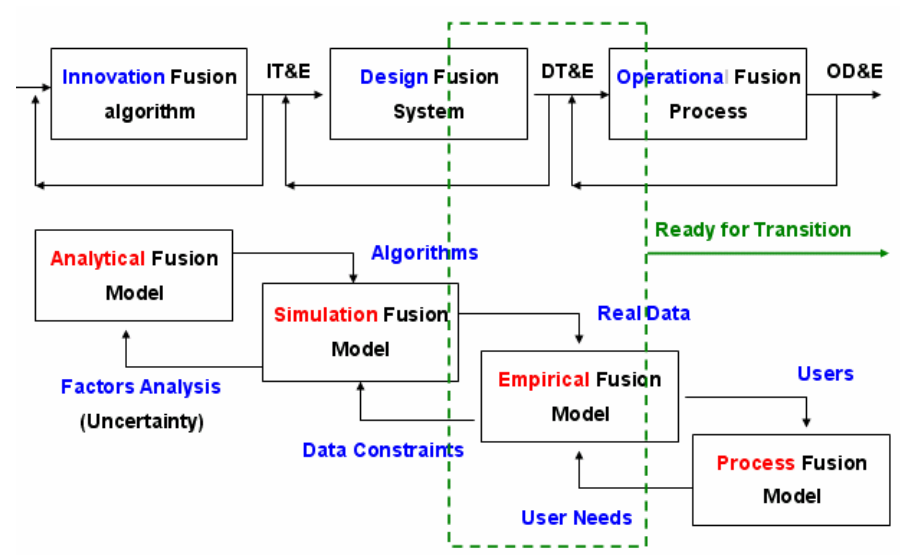

Figure 6: MTT Performance Assessment Process model for real world transition.

\section{CONCLUSIONS}

This position paper evaluated an MTT performance assessment from the standpoint of years of MTT research in designs, metrics, and simulated studies with the systematic view of transitioning these systems to real world capabilities. Whether the users are single sensor operators or part of a team, as shown in Figure 7, there is a need for system-level understanding of MTT performance. The ideas presented that support the transition of MTT systems to the real world include (1) enhanced scenarios delivered to the community by way of challenge problems and sensor, target, and environment models, (2) standardized metrics and appropriate methods for calculating the metrics, and (3) systematic testing of the complex system-level analysis through design of experiments to determine the sensitivity of the MTT solutions. With these ideas, the MTT community can manage user expectations, bound performance for sensor management, and determine the research areas of need for the future robust MTT success.
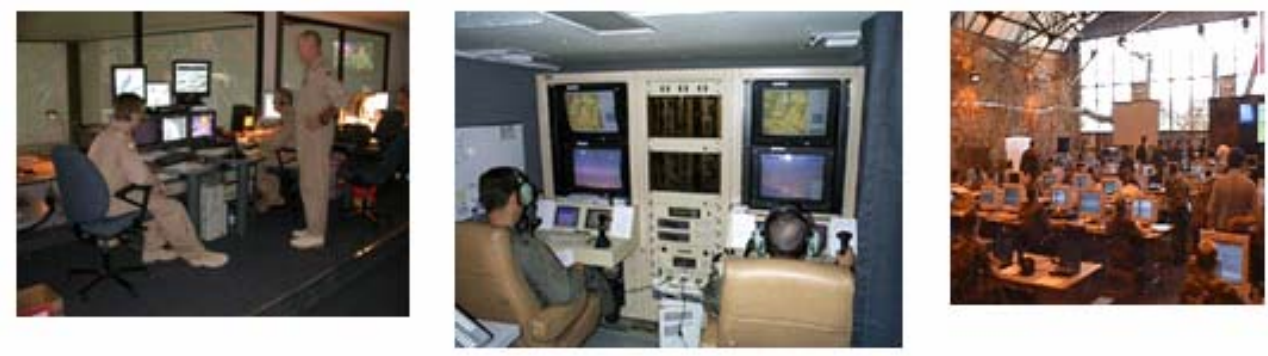

Figure 7: Users of MTT Systems requiring Performance Assessment.

\section{REFERENCES}

[1] E. Blasch, M. Pribilski, B. Daughtery, B. Roscoe, and J. Gunsett, "Fusion Metrics for Dynamic Situation Analysis,” Proc SPIE 5429, 2004.

[2] T. Kirubarajan, Y. Bar-Shalom, W. D. Blair, and G. A. Watson, "IMMPDAF for Radar Management and Tracking Benchmark with ECM," IEEE Trans. Aerospace and Electronic Systems, Vol 34, No. 4, Oct 1998.

[3] D. Blair, Metrics Briefing for Target Tracking, 2006.

[4] B. E. Fridling, "Performance Evaluation Methods for Multiple Target Tracking Algorithms," SPIE Vol. 1481, 1991.

[5] O. E. Drummond, "Performance Evaluation of Single Target Tracking in Clutter," SPIE Vol 2468, 1992.

[6] O. Drummond and B. E. Fridling, “Ambiguities in Evaluating Performance of Multiple Target Tracking Algorithms,” SPIE Vol. 1698, 1992

[7] O. E. Drummond, "Methodologies for Performance Evaluation of Multitarget Multisensor Tracking," SPIE Vol $3809,1999$.

[8] O. E. Drummond, W. D. Blair, G. C. Brown, T. L. Ogle, Y. Bar-Shalom, R. L. Copperman, and W. H. Barker, "Performance Assessment and Comparison of Various Tracklet Methods for Maneuvering Targets," SPIE Vol. 5096, 2003.

[9] S. Blackman, “Tracking System Performance Prediction and Evaluation,” Ch. 13 in Modern Tracking Systems, Artech House, 1999.

[10] S. Mori, C-Y. Chong, E. Tse, and E. P. Wishner, “Tracking and Classifying Multiple Targets Without A Priori Identification,” IEEE Trans. On Automatic Control, Vol AC-31, No. 5, May 1986. 
[11] C. Yang and Blasch, E. "Mutual-Aided Target Tracking and Identification,” Proc SPIE 5099, 2003.

[12] E. Blasch, "Modeling Intent for a Target Tracking and Identification Scenario," Proc SPIE, April 2004.

[13] E. Blasch, I. Kadar, J. Salerno, M. M. Kokar, S. Das, G. M. Powell, D. D. Corkill, and E. H. Ruspini, "Issues and Challenges of Knowledge Representation and Reasoning Methods in Situation Assessment (Level 2 Fusion)”, J. of Advances in Information Fusion, Dec. 2006.

[14] A. Steinberg, Information Fusion Tutorial, Fusion04, Stockholm, Sweden, 2004.

[15] R. L. Rothrock and O. E. Drummond, "Performance Metrics for Multiple-Sensor, Multiple-Target Tracking," SPIE Vol $4048,2000$.

[16] D. L. Hall, Mathematical Techniques in Multisensor Fusion, Chapter 7. Artech House, Norwood, MA, 1992.

[17] X. R. Li and Z. Zhao, "Evaluation of Estimation Algorithms Part 1: Incomprehensive Measures of Performance," IEEE Trans. Aerospace and Electronic Systems, Vol. 42, No. 4, Oct. 2006.

[18] S. Rawat, J. Llinas, and C. Bowman, "Design of Experiments for target Tracking Briefing,” 2004.

[19] O. E. Drummond, T. L. Ogle, and S. Waugh, “Metrics for Evaluating Track Covariance Consistency,” SPIE Vol. $6699,2007$.

[20] S. Rawat, J. Llinas, and C. Bowman, "Design of a Performance Evaluation Methodology for Data Fusion-Based Multiple Target Tracking Systems,” SPIE Vol. 5099. 2003.

[21] E. Waltz and J. Llinas, "System Modeling and Performance Evaluation," Chapter 11 in Multisensor Data Fusion, Artech House, 1990.

[22] P. Hanselman, C. Lawrence, E. Fortunano, B. Tenney, and E. Blasch, “Dynamic Tactical Targeting,” SPIE 04, 5441 , April 2004.

[23] K. C. Chang, T. Zhi and R. K. Saha, "Performance Evaluation of Track Fusion with Information Matrix Filter," IEEE Trans. Aerospace and Electronic Systems, Vol. 38, No. 2, April 2002.

[24] Y. Zhu, J. Zhoa, and K. Zhang, "Performance Analysis for Distributed Track Fusion with Feedback," Proc. $3^{\text {rd }}$ World Congress on Intelligent Control and Automation, 2000.

[25] V. Kaufmann, T. Ross, E. Lavely, and E. Blasch, "Score-Based SAR ATR Performance Model with Operating Condition Dependences," SPIE Vol. 6568, 2007.

[26] J. Johnson, “Analysis of Image Forming Systems”, Image Intensifier Symposium, Ft Belvoir, Va. Oct 6-7, 1958.

[27] B. Kahler, E. Blasch, D. Pikas, and T. Ross, "EO / IR ATR Model to Support Target Identification Fusion”, SPIE Vol 6566, 2007.

[28] T. D. Ross, J. J. Bradley, L. J. Hudson, M. P. O’Connor, “SAR ATR - So What's The Problem? - An MSTAR Perspective”, Proc. of SPIE, Vol. 3721, April 1999

[29] E. Blasch, E. Lavely and T. Ross "Fidelity Metric for SAR Performance Modeling” SPIE Vol 5808, April 2005.

[30] G. Arnold, T. Ross, L. Westerkamp, L. Carin, and R. Moses, “The ATR Center and ATRPedia,” SPIE Vol 6978, 2008.

[31] J. C. Mossing, T. D. Ross, “An Evaluation of SAR ATR Algorithm Performance Sensitivity to MSTAR Extended Operating Conditions”, Proc. of SPIE, Vol. 3370, April 1998.

[32] T. DeMarco Controlling Software Projects: Management, Measurement and Estimation, Yourdon Press, 1986

[33] M. T. Eismann, "Emerging Research Directions in Air-to-Ground Target Detection and Discrimination,” SPIE Vol. $5783,2005$.

[34] http://en.wikipedia.org/wiki/Quality_of_service

[35] M. E. Johnson and K. C. Chang, "Quality of Information for Data Fusion in Net Centric Publish and Subscribe Architectures,” Proc. Intl. Society of Information Fusion, 2005.

[36] I. Kadar, "Research Challenges in Network and Service Management for Distributed Net-Centric Fusion“, Invited Panel Session on Issues and Challenges in Resource Management with Applications to Real World Problems”, Organizers: Ivan Kadar and Ronald Mahler, Moderators: Ivan Kadar and Thia Kirubarajan, Proc. SPIE, Ivan Kadar Editor, Vol.6235, April 2006.

[37] E. Blasch and S. Plano, "Level 5: User Refinement to Aid the Fusion Process," Proc SPIE 5099, April 2003.

[38] E. Blasch, "Situation, Impact, and User Refinement," Proc SPIE 5096, April 2003.

[39] E. Blasch, I. Kadar, K. Hintz, J. Salerno, C. Chong, J. Salerno, and S. Das, "Resource Management Coordination with Level 2/3 Fusion”, IEEE AES Magazine , Dec. 2007.

[40] I. Kadar, "Distributed Multisensor Fusion with Network Connection Management”, Proc. SPIE, Vol. 5809, (Kadar Ed) April 2005.

[41] X. R. Li and Z. Zhao, "Relative Error Measures for Evaluation of Estimation Algorithms,” Proc. Intl. Society of Information Fusion, 2005.

[42] I. Kadar and L. Kurz, "Robustized Scalar Form of Gladyshev's Theorem with Applications to Non-Linear Systems," Proceedings of the Fourteenth Princeton Conference on Information Sciences and Systems, March 26-29, 1980

[43] I. Kadar and L. Kurz, "A Robustized Vector Recursive Stabilizer Algorithm for Image Restoration," Information and Control, Vol. 44, No. 3, March 1980.

[44] I. Kadar, "A Robustized Vector Recursive Algorithm in Estimation and Image Processing," Abstracts of papers, 1981 International Symposium on Information Theory, Santa Monica, CA, Feb. 9-12, 1981.

[45] I. Kadar "A Recursive Algorithm in Estimation and Image Processing," IBM Research Report, T. J. Watson Research Center, Yorktown Heights, NY, March 1981.

[46] I. Kadar, "Robust Tracking Novelty Filters Based on Linear Models", IEEE 1st Int. Conf. on Neural Networks, 1987.

[47] S. Arnborg, "Robust Bayesianism: Imprecise and Paradoxical Reasoning,” Conf. of Int. Society of Information Fusion, 2005.

[48] S. Arnborg, "Robust Bayesianism: Relation to Evidence Theory," Journal for Advances in Information Fusion, Vol 1, July 2006.

[49] L. Bai and E. Blasch, “Two-Way Handshaking Circular Sequential k-out-of-n Congestion System,” IEEE Journal of Reliability, Dec 2007.

[50] http://en.wikipedia.org/wiki/Design_of_Experiments.

[51] I. Kadar and L. Kurz, "A Class of Robust Edge Detectors Based on Latin Squares," Pattern Recognition, Vol. 11, No. 8/9, 1979.

[52] E. Blasch, “Assisted Target Recognition through User-Algorithm Fusion,” National Sensor and Data Fusion Conf., 2007. 
Can We Assess MTTs Like Consumer

- There are many multi-target tracking algorithms (car models)

- They are developed for different tracking applications - surface, ground,

air, missile (sports car, family cars, minivans, SUVs)

Consumer Reports Approach for Assessing Multi-target Trackers

Chee-Yee Chong

SPIE Panel on "Issues and Challenges in Performance Assessment of Multi-target Tracking Algorithms with Applications to Real-World

Problems"

March 17, 2008
- Users need help in

- Understanding how algorithms perform

- Selecting right algorithm for their application

- We need systematic approach to assess tracking algorithms (Annual Consumer Reports Issue on car buying)

\begin{tabular}{|c|c|}
\hline \multicolumn{2}{|c|}{ Car Reviews versus MTT Reviews } \\
\hline $\begin{array}{l}\text { - XXX Sedan } \\
\text { - System description } \\
\text { - Engine }-4 \text { cylinder } \\
\text { - Transmission - independent } \\
\text { - Brake - front disc/rear drum } \\
\text { - Steering - rack and pinion } \\
\text { - Component performance } \\
\text { - } 0 \text { to } 60 \mathrm{mph}-10 \mathrm{sec} \\
\text { - } 60 \text { to } 0 \mathrm{mph}-140 \mathrm{ft} \\
\text { - Top speed }-100 \mathrm{mph} \\
\text { - Cornering }-0.8 \mathrm{~g} \\
\text { - Performance on test range } \\
\text { - Time to complete }-30 \text { minutes } \\
\text { - Mpg }-40 \\
\text { - Performance on road or off-road } \\
\text { - Time to complete }-45 \text { minutes } \\
\text { - Mpg }-25\end{array}$ & $\begin{array}{l}\text { - YYY Multi-target Tracker } \\
\text { - Algorithm description } \\
\text { - Tracking filter - VSIMM } \\
\text { - Association - track-oriented MHT } \\
\text { - Component performance } \\
\text { - Filtering benchmark - xx m position } \\
\text { accuracy } \\
\text { - Association benchmark - yy\% } \\
\text { correct association } \\
\text { - Performance on simulated data }\end{array}$ \\
\hline ๑2008 BAE Systems. & \\
\hline
\end{tabular}

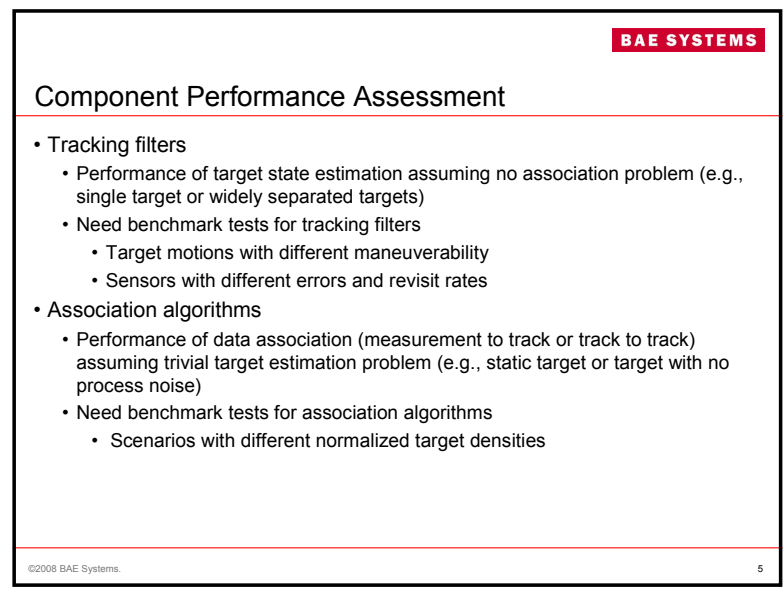

BAE SYSTEMS

Generic Multi-target Tracking Algorithm Architecture

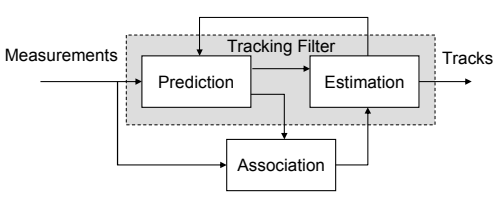

- Tracking filters

- Extended Kalman filter

- Interacting multiple models

- Particle filters

- Association algorithms

- Nearest neighbor

- Global nearest neighbor

- Multiple frame association / multiple hypothesis tracking

C2008 BAE Systems.

\section{BAE SYSTEMS}

Context Metrics to Characterize Problem Complexity

- Need simple parameters to characterize problem complexity

- Possible context metric for state estimation: target maneuvering index

$$
\begin{array}{ll}
\lambda=\frac{\sigma_{v} T^{2}}{\sigma_{w}} & \begin{array}{l}
\sigma_{v}: \text { Process noise standard deviation } \\
T: \text { Revisit interval }
\end{array} \\
\sigma_{w}: \text { Measurement noise standard deviation }
\end{array}
$$

- Possible context metric for association: normalized target density

$$
\beta=D_{y}|R|^{1 / 2} \quad \begin{aligned}
& D_{y}: \text { Density in measurement space } \\
& R: \text { Measurement error covariance }
\end{aligned}
$$




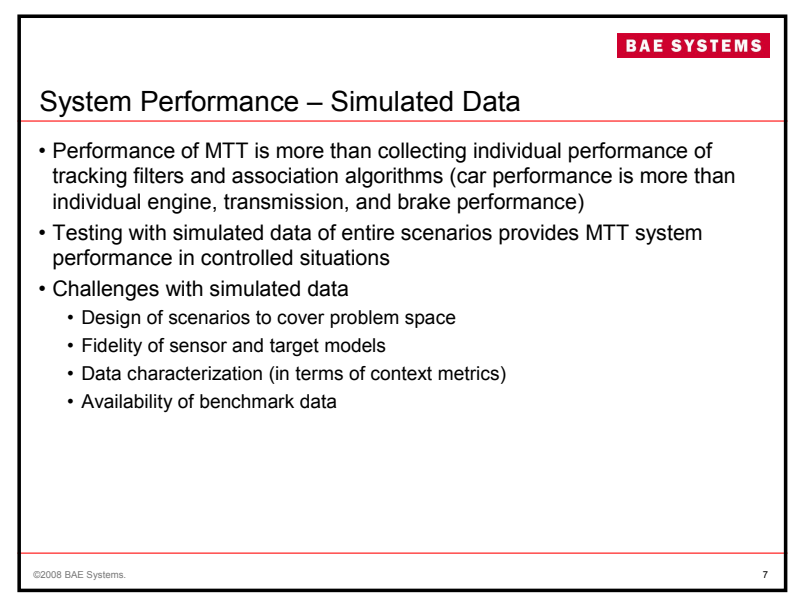

BAE SYSTEMS

Is It the Car or Is It the Road?

- Performance of MTT depends on algorithm itself and problem complexity

- Easy scenario - any algorithm will work

- Difficult scenario - no algorithm will work

- Context metrics should be displayed with performance metrics
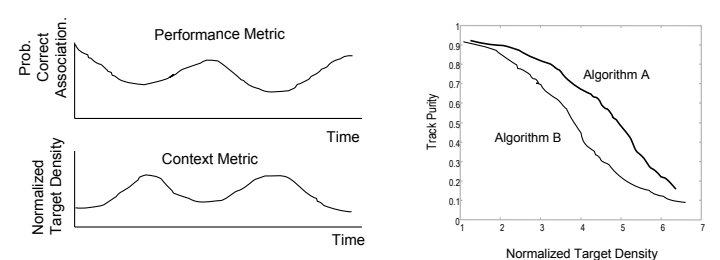

\begin{tabular}{|l|}
\hline BAE SYSTEMS \\
System Performance - Real Data \\
- Simulated data is not real (we need to drive on a REAL road) \\
- Testing algorithm with real data will reveal effects of imperfect target and \\
sensor models \\
- Challenges with real data \\
- Planning for data collection (targets, sensors, location, instrumentation) \\
- Limited data sets due to cost of collection \\
- Determining truth \\
- Assigning measurements to truth \\
\\
\end{tabular}

\begin{tabular}{|l|}
\hline BAE SYSTEMS \\
Summary \\
- Multi-target tracking algorithm performance assessment can learn from \\
Consumer Reports assessment of cars \\
- Need \\
- Component performance assessment \\
- System performance assessment with simulated data (driving on test tracks) \\
- System performance assessment with real data (driving on real roads) \\
- Challenges include \\
- Selecting performance metrics \\
- Designing benchmark test data \\
- Generating enough simulated data at right level of fidelity \\
- Collecting representative real data with ground truth \\
\end{tabular}




\title{
Consumer reports approach for assessing multi-target trackers
}

\author{
Chee-Yee Chong* \\ BAE Systems, 5050 El Camino Real, Suite 200, Los Altos, CA 94022
}

\begin{abstract}
This paper presents an approach for assessing multi-target trackers analogous to that used by Consumers Reports and car magazines in assessing cars. Car assessments usually contain a description of key components such as engine and brake, component performance such as acceleration and braking times, performance on test range, and performance on the road. The analogous assessment for trackers consists of a description of state estimation and association algorithms, performance of these individual components, system performance with simulated data, and system performance with real data.
\end{abstract}

Keywords: performance assessment, tracking filters, association algorithms

\section{INTRODUCTION}

Many civilian and military applications such as air traffic control and battlefield surveillance requires the tracking of multiple targets using data from one or more sensors. Since many multi-target tracking algorithms have been developed over the years, the user is faced with the problem of selecting the right algorithm from many alternative algorithms. More specifically, the user needs help in understanding the performance of the algorithms and selecting the right algorithm for the application.

This algorithm assessment and selection problem is quite similar to that of buying a car. There are many types of cars, e.g., sports cars, family cars, minivans, and sport utility vehicles (SUV's), analogous to different algorithms for tracking surface, ground, air, and space targets. However, car buying is now a fairly systematic process. For example, every April Consumer Reports publishes the annual car buying issue that contains information on basically all the cars sold in the United States. By consulting this issue, a car buyer can compare different cars and select one that meets his or her needs and budget. The objective of this paper is to describe a systematic approach for assessing tracking algorithms analogous to that for assessing cars. This assessment approach can potentially simplify the task of matching algorithms to applications.

The rest of this paper is organized as follows. Section 2 presents a structure for assessing multi-target tracking algorithms that is motivated by that used for assessing cars. Section 3 discusses the main components in a multi-target tracker (MTT) and the assessment of individual components. Section 4 describes system performance assessment with simulated and real data and the need to characterize the complexity of the problem.

\section{ASSESSMENT STRUCTURE}

A car review in Consumer Reports or car magazines such as Car and Driver or Road and Track usually has information on the basic components such as engine, transmission, brake, steering, and performance results on the individual components such as time from 0 to 60 miles per hour, braking distance from 60 to 0 miles per hour, etc. These component performance results are augmented by test results on both test ranges and under realistic driving conditions. A similar structure can be used for assessing multi-target trackers. The assessment will start with a description of the component algorithms such as those used for tracking and data association, and the performance of these component algorithms. These will be followed by system level performance with simulated data and real data such as those collected in flight tests. Table 1 compares the car reviews with MTT reviews.

*chee.chong@baesystems.com; phone 1 650-210-8822; fax 1 650-210-8824 
Table 1: Car reviews and MTT assessment have similar structures

\begin{tabular}{|c|c|}
\hline XXX Sedan & YYY Multi-target Tracker \\
\hline 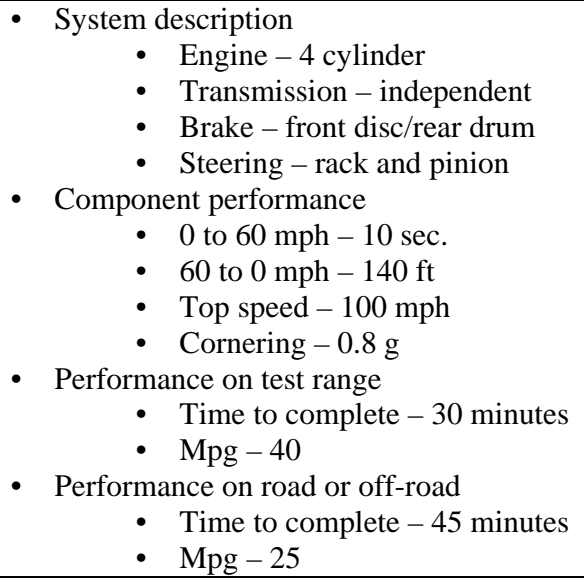 & 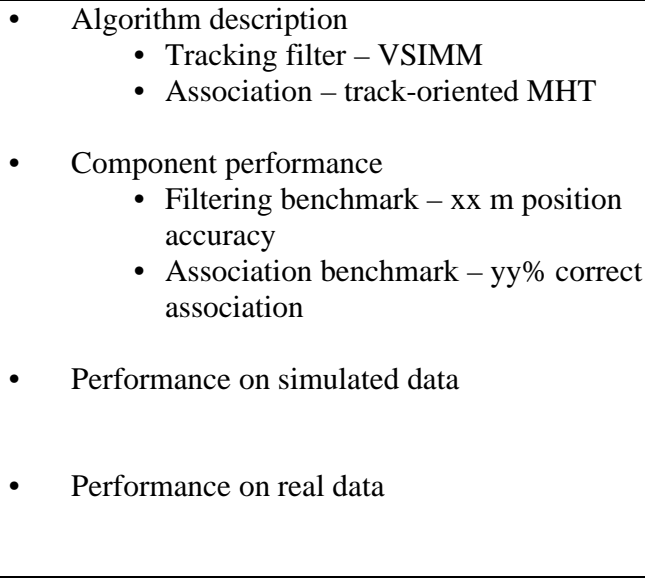 \\
\hline
\end{tabular}

\section{COMPONENT DESCRIPTION AND PERFORMANCE ASSESSMENT}

Performance assessment of multi-target trackers requires identifying the components in the algorithm and the performance of the individual components. With the exception of association free algorithms such as those described in [1], most multi-target tracking algorithms consist of the following steps (Figure 1): predicting the target states of the tracks to the reporting time, associating the reports to the tracks, and updating (estimating) the target states given the associated reports. Furthermore, prediction and estimation are usually implemented together in a tracking filter.

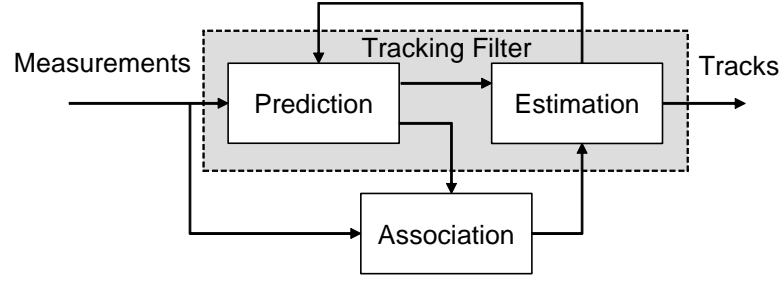

Figure 1: Generic MTT consists of tracking filter and association algorithms

Possible tracking filters include extended Kalman filters, interacting multiple models (IMM), and particle filters. Possible association algorithms include local nearest neighbor, global nearest neighbor, and multiple frame association or multiple hypotheses tracking.

Component performance assessment includes tracking filter performance assessment and association algorithm performance assessment. If the tracking filter in a MTT cannot be isolated for testing, its performance can be assessed by using data sets with no association problems, e.g., data from a single target or multiple widely separated targets. Benchmark tests for tracking filters should include target motions with different maneuverability and sensors with different errors and revisit rates. Similarly, association algorithms should be assessed stand alone with data sets with little difficulty in target state estimation, e.g., static target or targets whose models have no process noise. Benchmark tests for association algorithms should include scenarios with different normalized target densities.

Performance should be assessed for problems whose complexity can be parameterized easily. One way of characterizing complexity is by context metrics [2]. A possible context metric for state estimation difficulty is the target maneuvering index $\lambda=\sigma_{v} T^{2} / \sigma_{w}$ where $\sigma_{v}$ is the process noise standard deviation, $T$ is the revisit interval, and $\sigma_{w}$ is the measurement noise standard deviation. The equivalent context metric for association is normalized target density $\beta=D_{y}|R|^{1 / 2}$ where $D_{y}$ is the density in measurement space and $R$ is the measurement error covariance. 


\section{SYSTEM PERFORMANCE ASSESSMENT}

The performance of a system depends on how the components work together. This is true whether we are dealing with a car or a multi-target tracker. Thus, it is important to assess the performance of the system as a whole in addition to that of each individual component. System performance should be assessed under both highly controlled conditions (test range for a car and simulated data for MTT) and realistic conditions (actual roads for a car and real data for MTT).

Testing with simulated data allows performance assessment over large parameter spaces and is frequently the only way to find the performance envelope of an algorithm. Even though simulated data is generally cheaper to generate than real data, designing scenarios to cover enough interesting cases is still non-trivial. Target and sensor models are used to simulate the data. Highly detailed models produce realistic data but require significant processing sources and long simulated times. Thus selecting the appropriate model fidelity is very important. To facilitate algorithm assessment and reduce cost, the multi-target tracking community should develop benchmark data sets (e.g., [3]) for different tracking problems just like test tracks for testing cars.

Just as a car buyer wants to test drive the car on the real road, testing MTT with real data is very important because real data can reveal the effects of imperfect target and sensor models on the algorithm. Since real data collection is expensive, only limited amount of data can be collected. Thus it is important to carefully plan the target types and trajectories and the placement of the sensors. Instruments have to be placed on the targets to record accurate locations. Performance assessment of multi-target trackers requires assigning tracks to target. For simulated data, one can trace the origins of the measurements in the tracks to the targets and use them for the assignment. Since this tracing of measurements to targets is no longer possible in real data, another way of assigning tracks to targets has to be found.

Just as a high performance car cannot go fast on a poor road, the performance of MTT depends on the algorithm itself and problem complexity. Almost any algorithm will perform well on an easy scenario but almost any algorithm will fail for a difficult problem. Thus it is important to characterize the problem complexity when assessing algorithm performance (Figure 2).
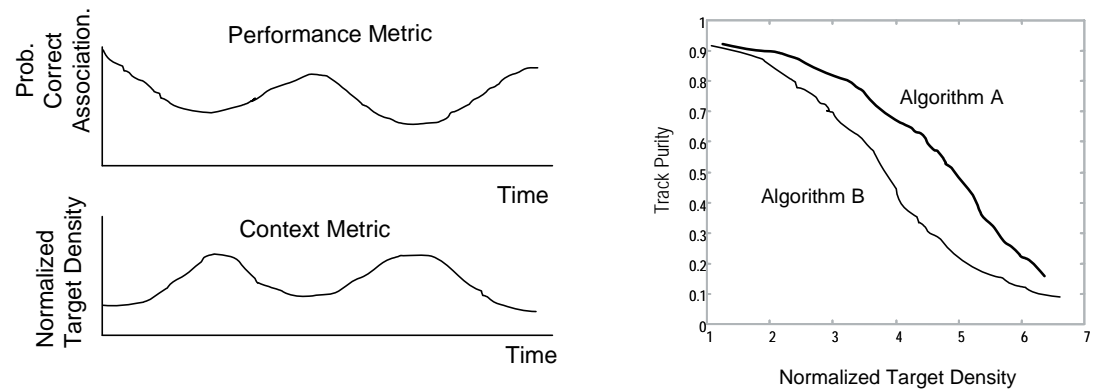

Figure 2: Both performance and context metrics should be displayed in performance assessment

\section{SUMMARY}

Multi-target tracking algorithm performance assessment can learn from Consumer Reports assessment of cars. Specifically, performance assessment should include component performance assessment, system performance assessment with simulated data (driving on test tracks), and system performance assessment with real data (driving on real roads). Challenges include selecting the appropriate performance metrics, characterizing problem space for testing (context metrics), developing benchmark test data, generating enough simulated data at right level of fidelity and collecting representative real data with ground truth

\section{REFERENCES}

[1] Mahler, R. P. Statistical Multisource-Multitarget Information Fusion, Artech House (2007)

[2] Chong, C-Y, "Problem characterization in tracking/fusion algorithm evaluation,” IEEE System Magazine, 12-17, (2001)

[3] Blair, W. D., and Watson, G. A., "Benchmark problem for radar resource allocation and target tracking in presence of ECM,” Naval Surface Warfare Center Dahlgren Division, NSWCDD/TR-96/10 (1996) 


\section{Challenges and Recent Advances in}

Estimation Performance Evaluation

\section{Rong Li}

Department of Electrical Engineering

University of New Orleans

New Orleans, Louisiana, U.S.A.

504-280-7416, xli@uno.edu

URL: http://ece.engr.uno.edu/li/

\section{Scope of Presentation}

- Tracking relies on estimation, but

- Tracking has its own issues

- We address

口 estimation performance directly,

a tracking and data fusion performance indirectly

that is, issues common to estimation, tracking, and data fusion performance evaluation

\section{Outline}

\section{- Background}

- Limitations and drawbacks of existing metrics

- Challenge 1: Comprehensive evaluation

- Challenge 2:

Evaluation without knowing ground truth

- Challenge 3: Algorithm comparison and ranking

- Summary

\section{Absolute Metrics vs. Others}

\begin{tabular}{l|l}
\hline Error Metric & Good for Evaluation of \\
\hline Absolute & Estimation system \\
\hline Relative & Estimation algorithm \\
\hline Frequency count & Hit-or-miss type application
\end{tabular}

- Metrics in use are absolute, don't meet diverse needs.

- We have presented two other classes of metrics: relative error metrics and frequency counts.

See: IEEE Trans. AES, 42(4):1340-1358, Oct. 2006

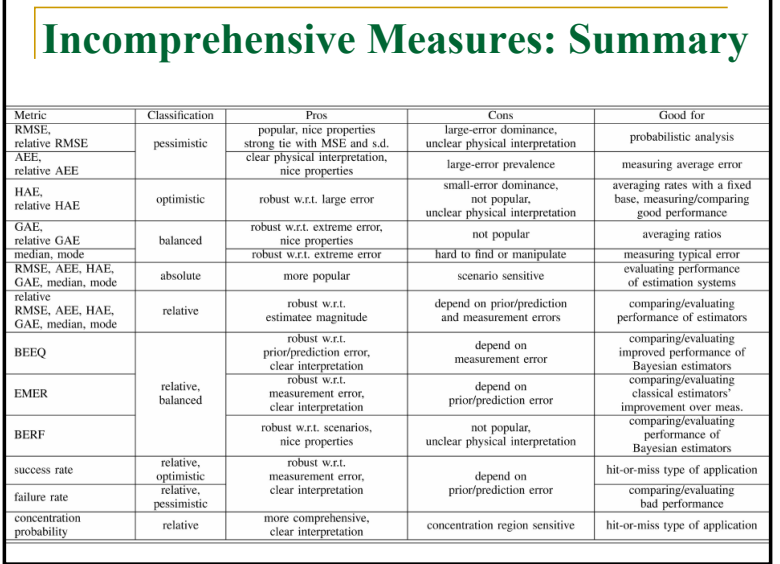

\section{Limitations of Existing Metrics}

- Need knowledge of ground truth

- Lack of comprehensive metrics:

Metrics abound, but few are comprehensive

- Metrics in use are absolute.

- Commonly used RMS error is seriously flawed:

口 It's very pessimistic:

- If all 100 errors are 1 except for one term of 1000 , then RMSE $\approx 100$

- What's RMSE of a filter if it diverges on one or few of many runs?

口 It has no clear physical interpretation:

Is it "distance" between estimatee and estimate? No!

- Conclusion:

Alternative metrics and better understanding are needed. 


\section{Classification of Metrics}

A performance metric is

- Pessimistic if it pays more attention to bad performance in that it consistently underestimates the performance (e.g. dominated by large errors)

- Optimistic if it pays more attention to good performance in that it consistently overestimates the performance (e.g., dominated by small errors)

- Neutralistic if it's neither pessimistic nor optimistic

- Balanced if its good performance and bad performance are accounted for in a balanced manner

The same concept applies to many other cases, e.g., estimators, trackers, fusers, and credibility measures.

\section{Challenge 1: Comprehensive Evaluation}

- Metrics in wide use are narrowly focused

- They are not fair, not good for performance ranking or comparison

- Our solutions:

- Error spectrum: aggregate most error metrics so far (Daum Tribute, May 2007)

口 Desirability metrics w.r.t. a desired error density (Daum Tribute, May 2007)

$\checkmark$ Concentration measures w.r.t. a reference density (Fusion'07)

\section{Error Spectrum}

Let $e=\|\tilde{x}\| /\|x\|$ or $\|\tilde{x}\|$ of estimation error $\tilde{x}$. Error spectrum is

$$
S(r)=S_{e}(r)=\left[E\left(e^{r}\right)\right]^{1 / r}, \quad r \in[-\infty, \infty]
$$

as a function of $r$. The lower $S(r)$ curve is the better

The most useful portion of the curve is: $-1 \leq r \leq 2$.

- $S(2)=$ RMSE, $S(1)=$ AEE, $S(0)=\mathrm{GAE}, S(-1)=$ HAE

- $\min \left\{e_{i}\right\}=S(-\infty) \leq S(r) \leq S(\infty)=\max \left\{e_{i}\right\}$

- $S(r)$ is increasing with $r: S(r)<S(s)$ if $r<s$

- $S_{e}(r)$ is linearly homogeneous in $e: S_{\alpha e}(r)=\alpha S_{e}(r), \forall \alpha>0$

- $S_{e}(r)$ is monotonic in e: $S_{e}(r) \leq S_{e^{\prime}}(r)$ if $e_{i} \leq e_{i}^{\prime}, \forall i$

- $S_{e}(r)$ is invariant if $e_{i}$ are permuted w.r.t. $i$

- $S_{e}(r)$ is strictly convex in $e$ for any fixed $r>1$

- $S_{e}(r)$ is strictly concave in e for any fixed $r<1$

\section{Evaluation with Unknown Ground Truth}

- Given:

- True data set $z_{1}, \ldots, z_{N}$, but don't know how it was generated

- Assumed (not the true) data model: $z \sim \hat{f}(z \mid x)$ or $z=\hat{h}(x, v)$

- For each data point $z_{i}$, obtain estimate $\hat{x}_{i}$

- Use the estimate and assumed model to generate mock data set $\hat{z}_{i 1}, \ldots, \hat{z}_{i M}$ :

- draw from $\hat{f}\left(z \mid \hat{x}_{i}\right)$ or

- generated by $\hat{z}_{i j}=\hat{h}\left(\hat{x}_{i}, v_{j}\right)$

- Measure distance between true data and mock data

$$
d(z, \hat{z})=\frac{1}{N} \sum_{j=1}^{N} d\left(z_{i}, \hat{z}_{i}\right), \quad d\left(z_{i}, \hat{z}_{i}\right)=\frac{1}{M} \sum_{j=1}^{M} d\left(z_{i}, \hat{z}_{i j}\right)
$$

\section{Challenge 2:}

Evaluation without Knowing Truth

- All existing metrics rely on knowing the ground truth

- Can we develop a performance evaluator that - Is comprehensive, systematic, generally applicable

- Doesn't need to know the ground truth?

- I thought the answer is NO for years

- Fortunately, I was wrong!

\section{Major Advantages of New Approach}

- Comprehensive

- Generally applicable

- No need to know the ground truth

- Accounts for model mismatch as well as estimation error

- Discourages either overfitting or underfitting

See: ONR-GTRI Workshop on Tracking and Sensor Fusion, May 2007 


\section{Challenge 3: Performance Ranking}

- How to rank performance of estimators?

- Rank them according to same measure

- Let them compete with each other: efficient

- We have developed (Fusion'07):

- Relative loss: quantify how much one loses to other

a Relative gain: quantify how much one gains over other

- Problem: transitivity may not hold

- Many other ways are possible

- Analogy: rank athlete performance

\section{Summary}

- Three challenges: comprehensive metric, unknown truth, performance ranking

- Existing methods known to us

- Are incomprehensive, absolute,

$\checkmark$ Rely on knowing the ground truth, or

- May ignore crucial coupling b/t estimate and estimatee

- A general approach to comprehensive evaluation of estimation performance without knowing the ground truth is possible

- Such an approach is outlined here

- Measure is not the only yardstick for performance ranking

- Rank by outcomes of competition

\section{Main References}

- X. R. Li, Z.-L. Zhao, "Evaluation of estimation algorithms. Part I: incomprehensive performance measures," IEEE Trans. AES, 42(4):1340-1358, Oct. 2006

- X. R. Li, Z.-L. Zhao, and Z.-S. Duan, "Error Spectrum and Desirability Level for Estimation Performance Evaluation," Proc. Of Workshop on Estimation, Tracking and Fusion: A Tribute to Fred Daum, Monterey, CA, USA, May 24, 2007

- X. R. Li, "Comprehensive Evaluation of Estimation Performance without Knowing Ground Truth," ONR-GTRI Workshop on Tracking and Sensor Fusion, May 2007

- Z.-L. Zhao and X. R. Li, "Two Classes of Relative Measures of Estimation Performance," Proc. 2007 International Conf. Information Fusion, Québec City, Canada, July 9-12, 2007, paper \# 1432 


\title{
Challenges and Recent Advances in Estimation Performance Evaluation ${ }^{*}$
}

\author{
X. Rong Li \\ Department of Electrical Engineering, University of New Orleans, New Orleans, LA 70148, USA \\ xli@uno.edu, 504-280-7416, 504-280-3950 (fax)
}

Outline. Limitations of existing metrics. Challenge 1: Comprehensive evaluation. Challenge 2: Evaluation with unknown ground truth. Challenge 3: Alternative ranking of performance. Summary.

\section{Existing Metrics' Limitations, Alternative Metrics, and Classification of Metrics}

Limitations of existing metrics. While performance metrics for target tracking abound, there are only a few for estimation, especially widely used ones. These metrics have the following limitations in common: They are not comprehensive in scope; they need (complete) knowledge about the ground truth; they are sensitive to scenarios of evaluation.

Almost all evaluations of estimation performance are centered on the so-called root-mean-square error $(\boldsymbol{R M S E})$, although it is more controversial as to which measures are better for estimator comparison (see, e.g. [1]). RMSE is seriously flawed: (a) It is very pessimistic: If all 100 errors are 1 except for one term of 1000 , then RMSE $\approx 100$. What is the RMSE of a filter if it diverges on one or few of many runs? (b) It has no clear physical interpretation, although it has a clear probabilistic interpretation. Many practitioners confuse RMSE with the distance between estimatee (quantity to be estimated) and estimate in our physical world. Unfortunately, this is not the case. Therefore, alternative metrics and better understanding are needed. As elaborated in [3], the widely used RMSE should be replaced by some other measures of performance in many cases.

Absolute error metrics, relative error metrics, and frequency counts. Virtually all existing metrics in use are absolute in that they are not with respect to any reference and thus depend critically on the evaluation scenario, including estimatee magnitude, data accuracy, and possibly accuracy of prior information. As such, their use for performance evaluation is justified for an entire estimation system (including an estimation algorithm, a measurement subsystem, and others) but not for an estimation algorithm per se. This exemplifies its insufficiency to address diverse needs. Instead, relative error metrics are much more suitable for evaluation of an estimation algorithm. Three classes of alternative measures were proposed in [3]: (a) absolute error metrics, including average Euclidean error (AEE), geometric average error (GAE), harmonic average error (HAE), median and mode of error norm; (b) relative error metrics, including Bayesian estimation error quotient (BEEQ), estimate-measurement error ratio (EMER), Bayesian error reduction factor (BERF), and relative RMSE, AEE, GAE, HAE, median, and mode; and (c) frequency counts, including success rate, failure rate, and concentration probability.

These three classes of metrics differ in domain of application and each is best for a variety of situations, as outlined in the next table. Individually, they also have their own pros and cons and serve different purposes. A table is available in [3] that summarizes pros and cons and intended applications of each and every one of these metrics.

\begin{tabular}{c|c}
\hline \hline Error Metric & Good for Evaluation of \\
\hline Absolute error metrics & Estimation system \\
\hline Relative error metrics & Estimation algorithm \\
\hline Frequency count & Hit-or-miss type application \\
\hline
\end{tabular}

Classification of metrics. To help better understand existing metrics and metrics to come, the following classification was proposed in [3] and made precise in [5]. A performance metric is pessimistic if it pays more attention to bad performance in that it consistently underestimates the performance (e.g. if it is dominated by large errors); optimistic if it pays more attention to good performance in that it consistently overestimates the performance; neutralistic if it's neither pessimistic nor optimistic; and balanced if its good performance and bad performance are accounted for in a balanced manner. The same concept applies to many other matters, e.g., classification of estimators, trackers, fusers, and credibility measures [4].

\footnotetext{
${ }^{*}$ Research supported in part by Navy through Planning Systems Contract \# N68335-05-C-0382 and Project 863 through grant 2006 AA01Z126.

${ }^{\dagger}$ Target tracking relies on estimation, but tracking has its own issues. Estimation is in the core of data fusion, although each includes components not covered by the other. Although we choose to deal with estimation performance directly and explicitly here, what is presented addresses tracking and data fusion performance indirectly. In other words, we are actually talking about issues common to estimation, tracking, and data fusion performance evaluation.
} 


\section{Challenge 1: Comprehensive Evaluation of Performance}

Metrics in wide use such as RMSE have a narrow focus. They are not fair nor good for performance ranking or comparison. To alleviate this problem, several more comprehensive metrics have been proposed in four classes: (a) error spectrum, which aggregates most error metrics available; (b) desirability metrics, which measure how close the actual error is to a desired error density; (c) concentration measures, which quantify how concentrated the errors are w.r.t. a reference density; and (d) the comprehensive estimation performance evaluator of the next section, which does not need to know the ground truth.

Error spectrum. Let $e=\|\tilde{x}\|$ or $e=\|\tilde{x}\| /\|x\|$ be the absolute or relative estimation error norm. The error spectrum (ES) introduced in [5] is defined as

$$
S(r)=S_{e}(r)=\left[E\left(e^{r}\right)\right]^{1 / r}
$$

ES has a large array of nice properties important for performance evaluation, including (a) it aggregates many of the above metrics (e.g., $S(2)=$ RMSE, $S(1)=\mathrm{AEE}, S(0)=\mathrm{GAE}, S(-1)=\mathrm{HAE}, S(\infty)=\max e$ and $S(-\infty)=\min e$ ); (b) min $e \leq S(r) \leq \max e$; (c) $S(r)$ is strictly increasing with $r$; (d) $S_{e}(r)$ is monotonic and linearly homogeneous in $e$; (e) $S_{e}(r)$ is invariant w.r.t. any label system for $e$; and (f) $S_{e}(r)$ is strictly convex in $e$ for any fixed $r>1$ and strictly concave in $e$ for any fixed $r<1$.

It turns out that [5] error spectrum $S(r)$ is pessimistic if $r>0$, optimistic if $r<0$, and balanced if $r=0$; the (absolute and relative) RMSE, AEE, and the failure rate are pessimistic; the (absolute and relative) HAE and success rate are optimistic; the (absolute and relative) GAE, median error, error mode, and the BEEQ, EMER, and BERF are neutralistic.

Desirability measures. They measure closeness between the actual error density $f(\cdot)$ and desired error density $f_{d}(\cdot)$. Several closeness measures have been proposed based on the correlation coefficient, Wasserstein metric, Kullback-Leibler information [6], and total variation distance. For example, the one based on the correlation coefficient, called desirability level [5] is defined as $\rho(0)$, where

$$
\rho(u)=\frac{\int f_{d}(\tilde{x}+u) f(\tilde{x}) d \tilde{x}}{\left[\int f_{d}(\tilde{x}+u)^{2} d \tilde{x} \int f(\tilde{x})^{2} d \tilde{x}\right]^{1 / 2}}
$$

It has some nice properties, including positive definiteness, invariance under invertible affine transformations, and having the standard range: $0 \leq \rho(0) \leq 1$.

Concentration measures. They quantify concentration of the actual error relative to a reference distribution [7]. One of them, called relative concentration index (RCI), is defined as $\mathrm{RCI}=E\left[f_{r}(\tilde{x}) \mid f\right] / E\left[f_{r}(\tilde{x}) \mid f_{r}\right]$, where $f$ and $f_{r}$ are the actual and reference error densities, respectively. These measures have some nice properties and can also serve as measures for performance comparison. For example, a distribution is more concentrated than the reference if and only if RCI $>1$.

\section{Challenge 2: Evaluation with Unknown Ground Truth}

Most, if not almost all, people believe that performance evaluation cannot be done without knowing the ground truth. How would we know how large the estimation error is without knowing the truth? Indeed, all existing metrics developed so far rely on this knowledge. Computer simulation based performance evaluation can largely get by with this limitation. It, however, does pose a serious challenge for other types of studies, especially those using real-world data, in which case we struggle to come up with an accurate enough knowledge of the ground truth. Everyone who did such a study should have this experience.

A grand challenge is then: Is it possible to evaluate performance without the need to know the ground truth? Even better, can this be done comprehensively, systematically, and with general applicability? Most people think it impossible. Fortunately, this is not the case!

Comprehensive estimation performance evaluator $($ CEPE $)$. Here is a concrete evaluator that makes the above dream come true [2]: (a) Given true data set $z_{1}, \ldots, z_{N}$ without knowledge of how it was generated, and assumed (not necessarily correct) data model: $z \sim \hat{f}(z \mid x)$ or $z=\hat{h}(x, v)$. (b) For each data point $z_{i}$, obtain estimate $\hat{x}_{i}$. (c) Use the estimate and the assumed model to generate mock data set $\hat{z}_{i 1}, \ldots, \hat{z}_{i M}$ : draw from $\hat{f}\left(z \mid \hat{x}_{i}\right)$ or generated by $\hat{z}_{i j}=\hat{h}\left(\hat{x}_{i}, v_{j}\right)$. (d) Measure distance between the true data and the mock data

$$
d(z, \hat{z})=\frac{1}{N} \sum_{j=1}^{N} d\left(z_{i}, \hat{z}_{i}\right), \quad d\left(z_{i}, \hat{z}_{i}\right)=\frac{1}{M} \sum_{j=1}^{M} d\left(z_{i}, \hat{z}_{i j}\right)
$$

A more powerful version of this evaluator particularly good for state estimation has also been developed.

Major advantages. It is comprehensive, systematically, and generally applicable; it does not need to know the ground truth; it accounts for model mismatch as well as estimation error; it discourages either overfitting or underfitting. 


\section{Challenge 3: Alternative Ranking of Performance}

Given multiple estimators, how do we rank their performance? A commonly used approach is to rank them according to the same measure of performance. This is natural and most people stick to it thinking there are no viable alternatives.

In fact, to determine their ranking, we may let them compete with one another [1]. This alternative is distinctive in its high efficiency. While ranking based on a common performance measure depends only on information that is "marginal" of each estimator, this alternative uses directly "joint" or relative information of the multiple estimators. One may argue that usually a single estimator is used in an application and so the joint or relative information of the multiple estimators should not play any role in performance evaluation. To answer this criticism, consider the analogy of selecting a capable person for a challenging task by an IQ contest. Here the winner will also have to accomplish the task alone, yet selection by a contest is sensible and in fact common practice. The rationale lies largely in the high efficiency of this alternative.

In view of the above, we have developed [7] several measures in this category, including the relative loss measure, which quantifies how much worse whenever one estimator loses to the other, and the relative gain measure, which quantifies how much better whenever one estimator beats the other, both over many pairwise competitions.

A problem with these measures is that transitivity does not hold in general (e.g., $A$ beats $B$ and $B$ beats $C$ does not guarantee $A$ beats $C$ ). Another rich class of alternative measures in the same spirit is under development. It appears that many of them can guarantee transitivity. Study of other alternatives is worthwhile and actually overdue. Here it is certainly beneficial to consider the analogy of ranking performance of athletes.

\section{Summary}

Existing methods and metrics for estimation performance evaluation have serious limitations. They are narrowly focused, overly pessimistic, hyper sensitive to evaluation scenario, totally reliant on knowing the ground truth, and may ignore crucial coupling between the estimate and estimatee.

Three challenges have been articulated: development of a comprehensive metric of estimation performance, evaluation of estimation performance without the need to know the ground truth, and development of alternative methods and measures for estimation performance ranking.

A general approach to comprehensive evaluation of estimation performance without the need to know the ground truth is possible. Such an approach has been developed and is outlined here.

Various metrics in three classes have been proposed recently: absolute metrics, relative metrics, and frequency counts. Having distinctive pros and cons, they should take over the widely used RMSE in many cases.

Three other classes of metrics have been developed: error spectrum, desirability metrics, and concentration measures. They are much more comprehensive than existing ones.

The use of a common performance measure is not the only yardstick for performance ranking. Alternatives do exist and may prove superior. A viable one is to rank by outcomes of competitions among estimators. Two measures in this category have been proposed and a third class is under development.

\section{References}

[1] J. P. Keating, R. L. Mason, P. K. Sen, and P. K. Seri. Pitman's Measure of Closeness: A Comparison of Statistical Estimators. SIAM, Philadelphia, 1993.

[2] X. R. Li. Comprehensive Evaluation of Estimation Performance without Knowing Ground Truth. In ONR-GTRI Workshop on Tracking and Sensor Fusion, Monterey, CA, USA, May 2007.

[3] X. R. Li and Z.-L. Zhao. Evaluation of Estimation Algorithms-Part I: Incomprehensive Performance Measures. IEEE Trans. Aerospace and Electronic Systems, AES-42(4):1340-1358, Oct. 2006.

[4] X. R. Li and Z.-L. Zhao. Measuring Estimator's Credibility: Noncredibility Index. In Proc. 2006 International Conf. on Information Fusion, Florence, Italy, July 2006.

[5] X. R. Li, Z.-L. Zhao, and Z.-S. Duan. Error Spectrum and Desirability Level for Estimation Performance Evaluation. In Proc. of Workshop on Estimation, Tracking and Fusion: A Tribute to Fred Daum, Monterey, CA, USA, May 2007.

[6] Z.-L. Zhao and X. R. Li. Probabilistic Model Distortion Measure and Its Application to Model-Set Design of Multiple Model Approach. In Proc. 38th Asilomar Conf. on Signals, Systems and Computers, pages 2146-2150, Pacific Grove, CA, USA, November 2004.

[7] Z.-L. Zhao and X. R. Li. Two Classes of Relative Measures of Estimation Performance. In Proc. 2007 International Conf. on Information Fusion, Qubec City, Canada, July 2007. 


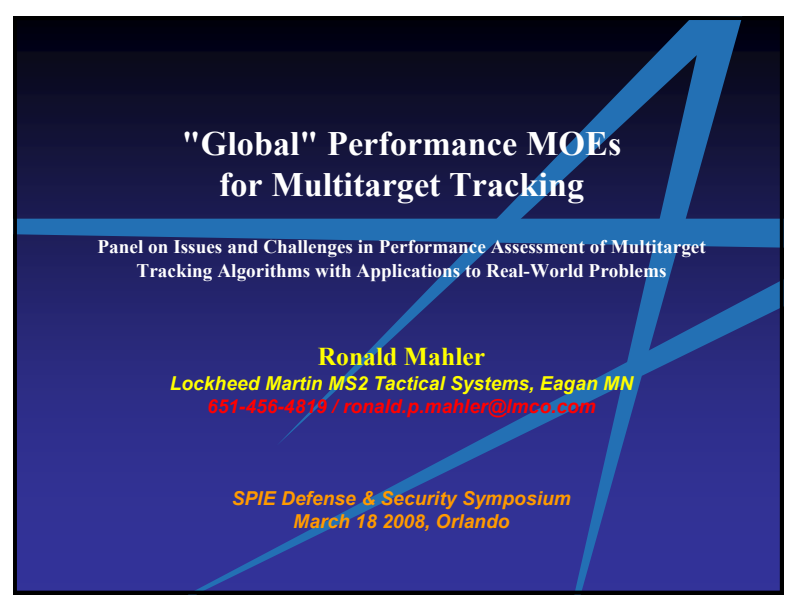

Multi-Object Csiszár Discrimination

Mahler (1997)

- determine "global" MoEs for multitarget fusion algorithms

- based on Csiszar information-discrimination "distances"

$$
\text { convex kernel }
$$
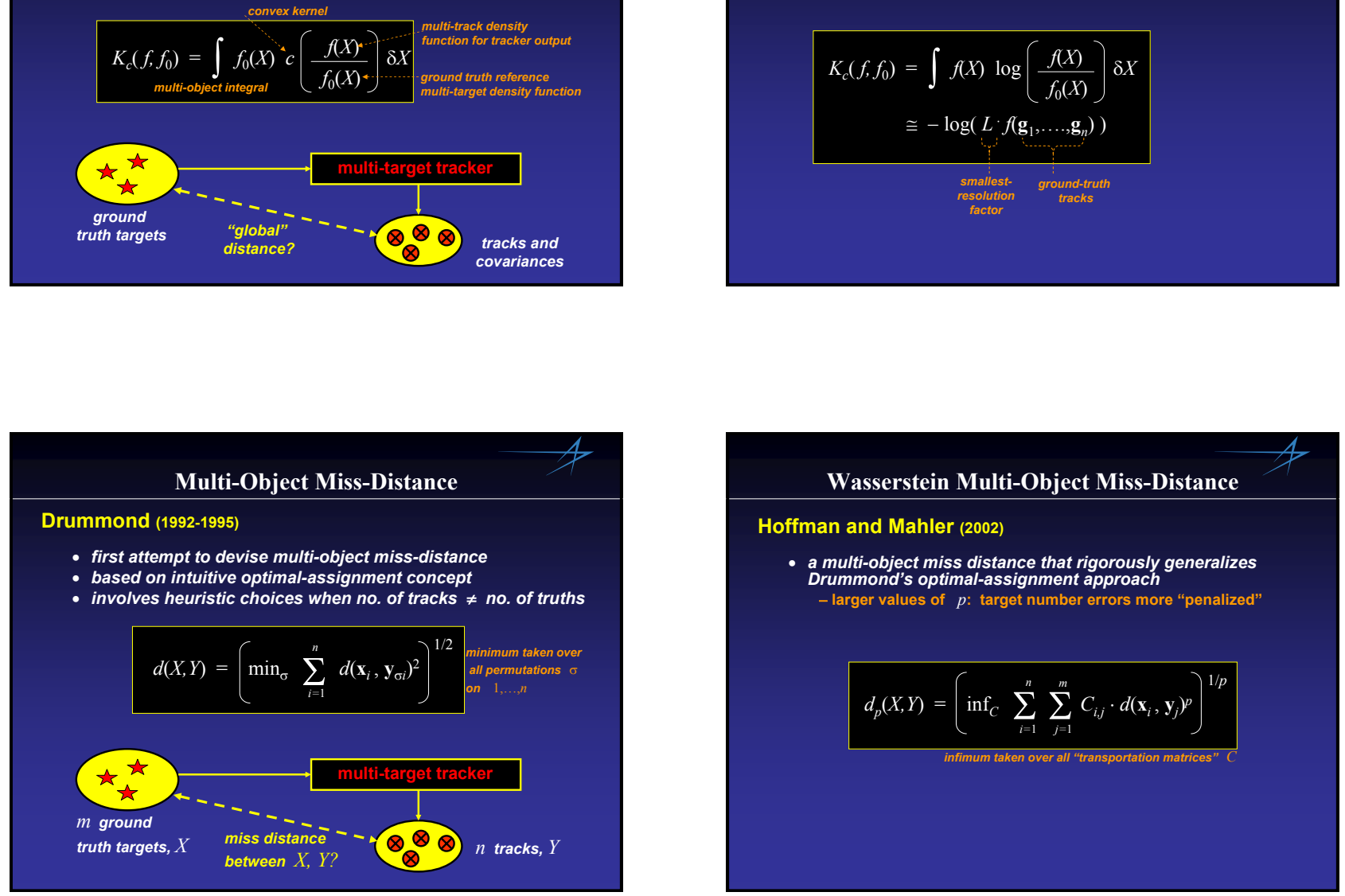

\section{Multi-Object Kullback-Leibler Discrimination}

\section{Mahler (1997)}

- special case: $c(x)=x \cdot \log x$

- well-known information-theoretic measure of effectiveness

$$
\begin{aligned}
K_{c}\left(f, f_{0}\right) & =\int f(X) \log \left(\frac{f(X)}{f_{0}(X)}\right) \delta X \\
& \cong-\log \left(L \cdot f\left(\mathbf{g}_{1}, \ldots, \mathbf{g}_{n}\right)\right)
\end{aligned}
$$

$$
\begin{gathered}
\text { smallest- } \\
\text { resolution } \\
\text { factor }
\end{gathered} \quad \begin{gathered}
\text { ground-truth } \\
\text { tracks }
\end{gathered}
$$

Iviii 

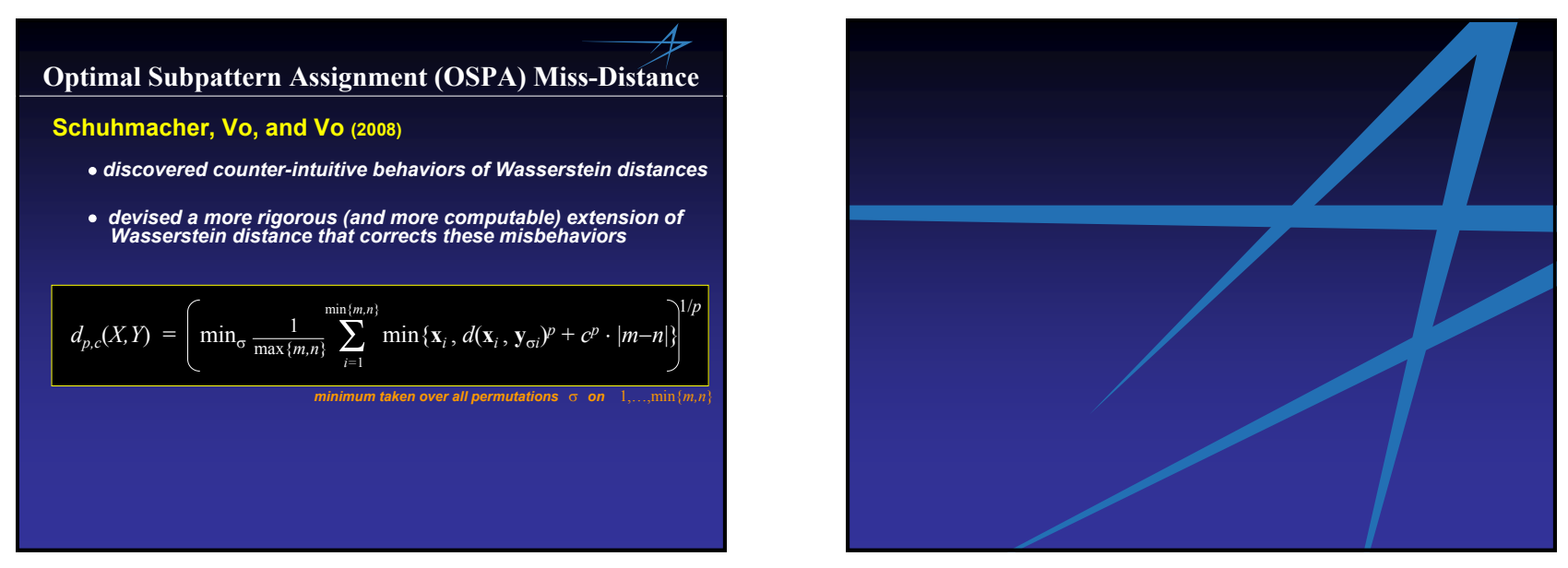


\title{
"Global" Performance MOEs for Multitarget Tracking
}

\author{
Position Paper: Panel on Issues and Challenges in Performance Assessment of Multitarget \\ Tracking Algorithms with Applications to Real-World Problems
}

\author{
Ronald Maher \\ Lockheed Martin MS2 Tactical Systems, Eagan MN
}

February 20, 2008

Traditionally, performance analysis of multitarget data fusion algorithms has been based on measures of effectiveness (MOEs) that quantify "local" aspects of performance: probability of target detection, average localization miss distance, probability of correct ID, etc. Because multitarget tracking algorithms are highly nonlinear, improving performance with respect to one local MOE can result in decreasing performance with respect to another-and thus conflicting MOEs. Because of such problems, attempts have been made to construct over-all ("global") MOEs via, for example, heuristic weighted averages of local MOEs. Unfortunately, this approach suffers from its own difficulties. The resulting single MOEs have tended to be arbitrary, non-intuitive, difficult to interpret, and sometimes not even invariant with respect to a change of units of measurement.

This position paper argues that comprehensive multitarget tracking performance estimation must be based on the fundamental multitarget statistics of a given tracking problem, as described by the theory of finite-set statistics. ${ }^{5}$ Specifically, truly comprehensive MOE should be defined in terms of the multitarget Bayes posterior probability distribution that characterizes the problem. I briefly survey the most promising techniques: multitarget Kullback-Leibler MOEs and multitarget Czsisár information-theoretic MOEs. I also survey intermediately comprehensive MOEs: the multitarget miss distances.

Comprehensive multitarget MOEs can be defined in terms of information theory. Let $f_{k \mid k}(X)$ be the multitarget probability distribution describing the output of a multitarget tracking algorithm. Then the family of multitarget Czsisár discrimination MOEs is defined by

$$
I^{c}(f, g) \triangleq \int f_{0}(X) \cdot c\left(\frac{f_{k \mid k}(X)}{f_{0}(X)}\right) \delta X
$$

where $f_{0}(X)$ is a multitarget reference density and where $c(x) \geq 0$ is a convex kernel function, i.e., $c(1)=0$ and $c(x)$ is strictly convex at $x=1$. Examples are $c(x)=x \cdot \log x$ and $c(x)=(\sqrt{x}-1)^{2}$ and $c(x)=(x-1)^{2}$. If we choose the first of these and let $f_{0}(X)=f_{\text {Grnd }}(X)$ model ground truth $\mathbf{g}_{1}, \ldots, \mathbf{g}_{\gamma}$, then we get

$$
K\left(f_{k \mid k}, f_{\text {Grnd }}\right)=\int f_{k \mid k}(X) \cdot \log \left(\frac{f_{k \mid k}(X)}{f_{\text {Grnd }}(X)}\right) \delta X \cong-\log L^{-1} f_{k \mid k}\left(\left\{\mathbf{g}_{1}, \ldots, \mathbf{g}_{\gamma}\right\}\right)
$$

where $L$ is the (hyper)volume of the smallest degree of localization resolution that is desired for the given problem. Such MOEs were introduced in Chapter 8 of Mathematics of Data Fusion. ${ }^{3}$ They have been used to develop MOEs for multitarget tracking algorithms, for sensor management algorithms, and other applications. ${ }^{2,7,10,1,6,9}$

Another type of MOE is not as comprehensive as information-theoretic MOEs, but is in some ways more intuitively accessible. These are the multitarget miss distances. Hoffman and Mahler ${ }^{4}$ introduced the family of 
Wasserstein distances. Let $X=\left\{\mathbf{x}_{1}, \ldots, \mathbf{x}_{n}\right\}$ and $Y=\left\{\mathbf{y}_{1}, \ldots, \mathbf{y}_{m}\right\}$ where $|X|=n$ and $|Y|=m$. Then the Wasserstein distance of power $p$ is defined by

$$
d_{p}(X, Y) \triangleq \inf _{C} \sqrt[p]{\sum_{i=1}^{n} \sum_{j=1}^{m} C_{i, j} \cdot d\left(\mathbf{x}_{i}, \mathbf{y}_{j}\right)^{p}}
$$

where the infimum is taken over all $n \times m$ so-called "transportation matrices" $C$. The larger the value of $p$, the more that $d_{p}^{W}\left(X, X^{\prime}\right)$ penalizes deviations in target number.

Recently, Schuhmacher, Vo, and $\mathrm{Vo}^{8}$ have uncovered a number of deficiencies in the Wasserstein distances when used for assessing the performance of multitarget tracking algorithms. In response, they have devised closely related but even more theoretically and practically justifiable multitarget miss distances called the optimal sub-pattern assignment (OSPA) distances. Let $c>0$. If $n \leq m$ these are defined by

$$
d_{p}^{c}(X, Y) \triangleq \sqrt[p]{\min _{\sigma} \frac{1}{m} \sum_{i=1}^{n} \min \left\{\mathbf{x}_{i}, d\left(\mathbf{x}_{i}, \mathbf{y}_{\sigma i}\right)^{p}+c^{p} \cdot(m-n)\right\}}
$$

where the minimum is taken over all permutations $\sigma$ on $1, \ldots, m$. If $n \geq m$ then $d_{p}^{c}(X, Y) \triangleq d_{p}^{c}(Y, X)$.

\section{REFERENCES}

[1] A. El-Fallah, J. Hoffman, T. Zajic, R. Mahler, and R. Mehra, "Scientific performance evaluation for distributed sensor management and adaptive data fusion," SPIE Proc Vol. 4380, pp. 328-338, 2001.

[2] A. El-Fallah, R. Mahler, T. Zajic, E. SPIE Procin I. Kadar (ed.), Sign. Proc., Sensor Fusion, and Targ. Recog. IX, pp. 183-194, SPIE Vol. 4052, pp. 183-194, 2000.

[3] I. R. Goodman, R. P. S. Mahler, and H. T. Nguyen, Mathematics of Data Fusion, Kluwer Academic Publishers, New York, 1997.

[4] J. Hoffman and R. Mahler, "Multitarget miss distance via optimal assignment," IEEE Trans. Sys., Man, and Cybernetics - Part A, vol. 34, no. 3, pp. 327-336, 2004.

[5] R. Mahler, Statistical Multisource-Multitarget Information Fusion, Artech House, Norwood, MA, 2007.

[6] J. Hoffman, R. Mahler, and T. Zajic, "User-defined information and scientific performance evaluation," SPIE Proc Vol. 4380, pp. 300-311, 2001.

[7] A. El-Fallah, R. Mahler, T. Zajic, E. Sorensen, M. Alford, and R. Mehra, "Scientific performance evaluation for sensor management," SPIE Proc Vol. 4052, pp. 183-194, 2000.

[8] D. Schuhmacher, B.-T. Vo, and B.-N. Vo, "A consistent metric for performance evaluation of multi-object filters," submitted for publication.

[9] T. Zajic and R. Mahler (1999) "Practical information-based data fusion performance evaluation," SPIE Proc. Vol. 3720, pp. 93-103, 1999.

[10] T. Zajic, J. Hoffman, and R. Mahler, "Scientific performance metrics for data fusion: new results," SPIE Proc. Vol. 4052, pp. 172-182, 2000. 

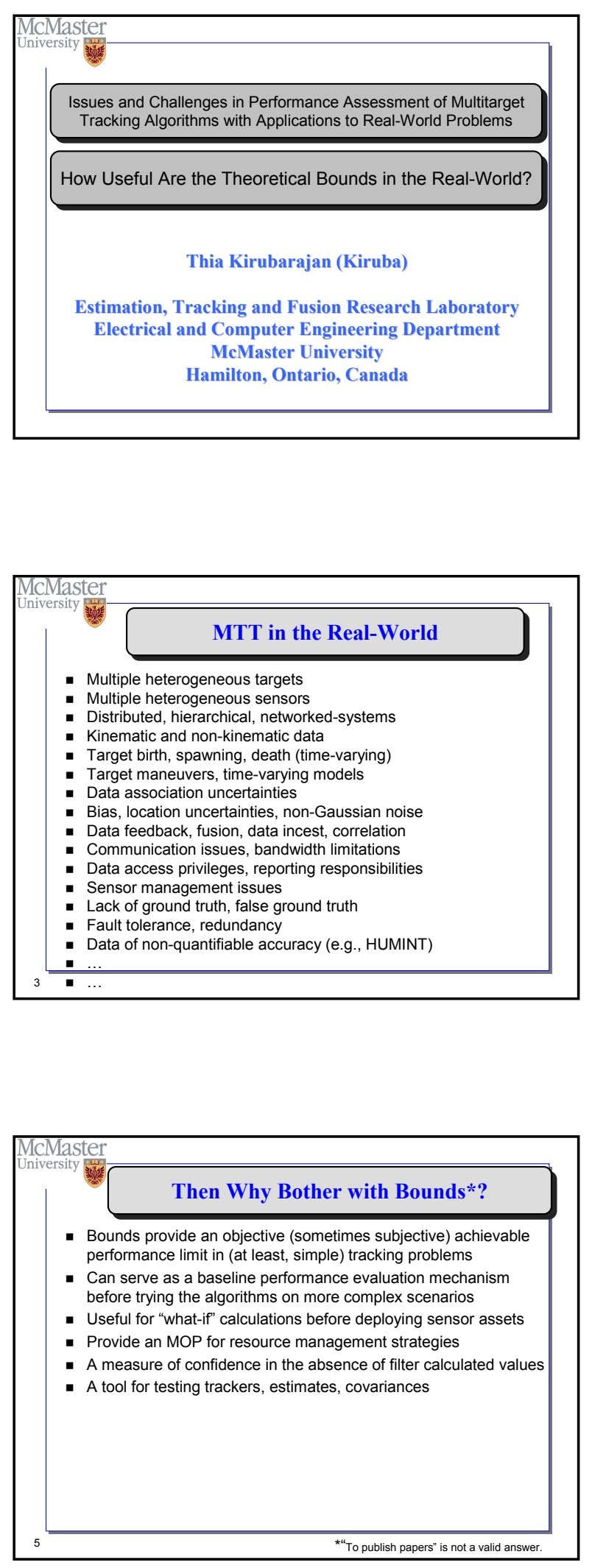

- Quantify analytically the best estimation/tracking accuracy
achievable by any tracker
- Done even before the first measurement is received
- Independent of particular measurement values and track
estimates
- Can be done offline
- Example: Cramer-Rao lower bound (CRLB), Weiss-Weinstein
bound (WWLB), Bhattacharya bound,
- The performance limit given by these is a bound and
not the bound

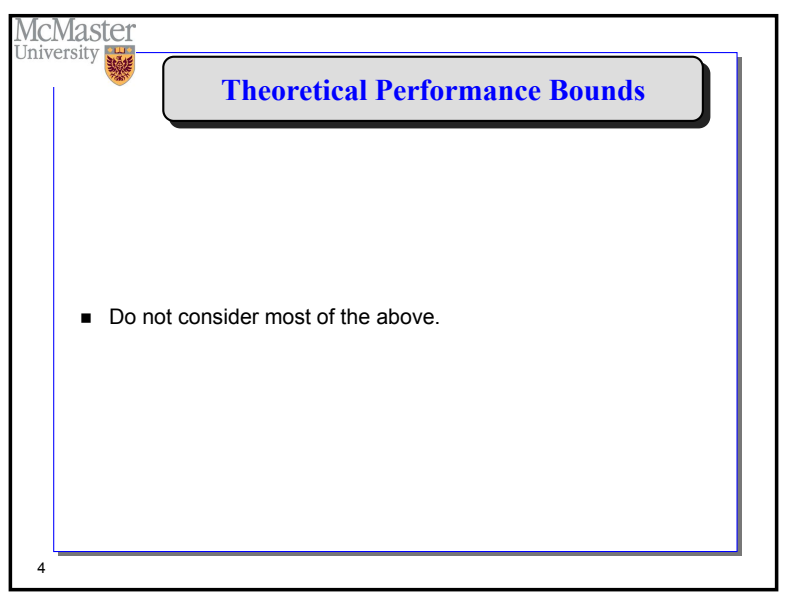



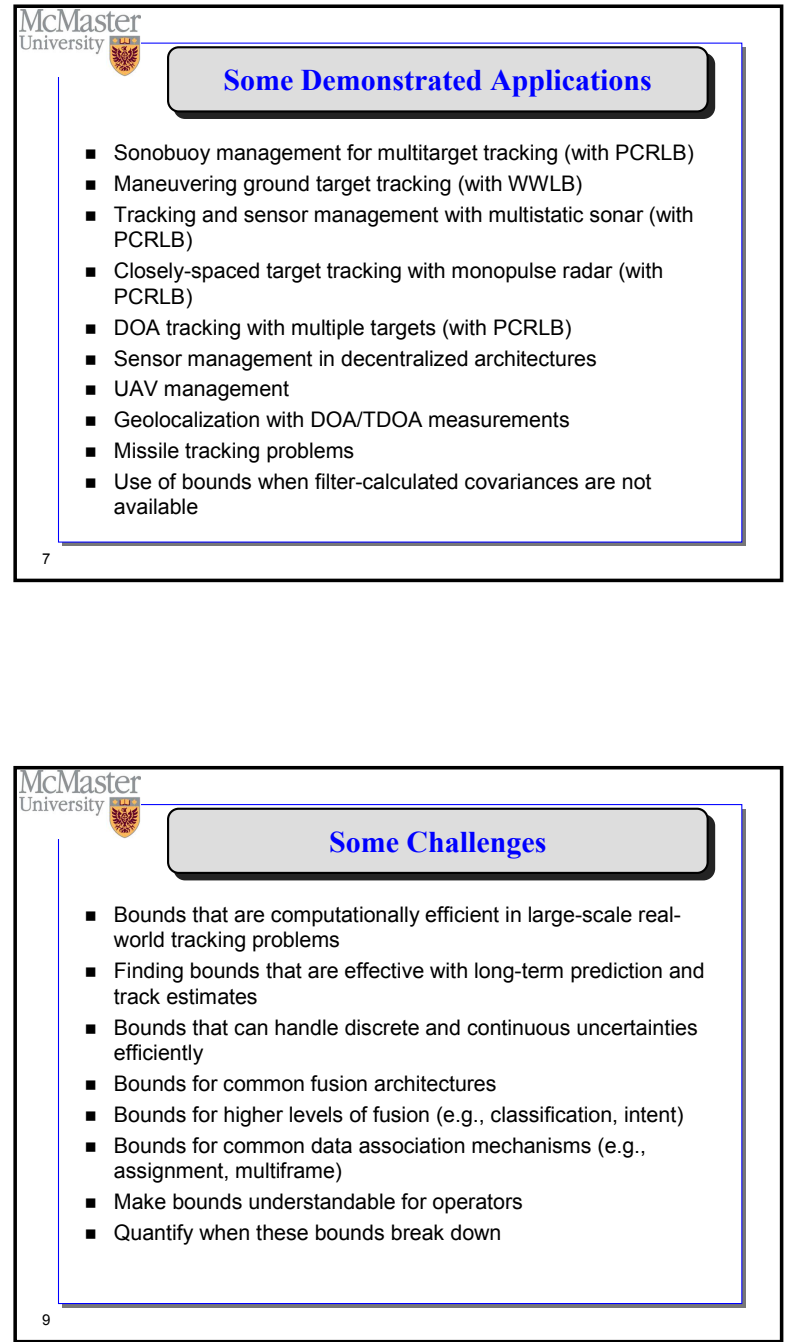
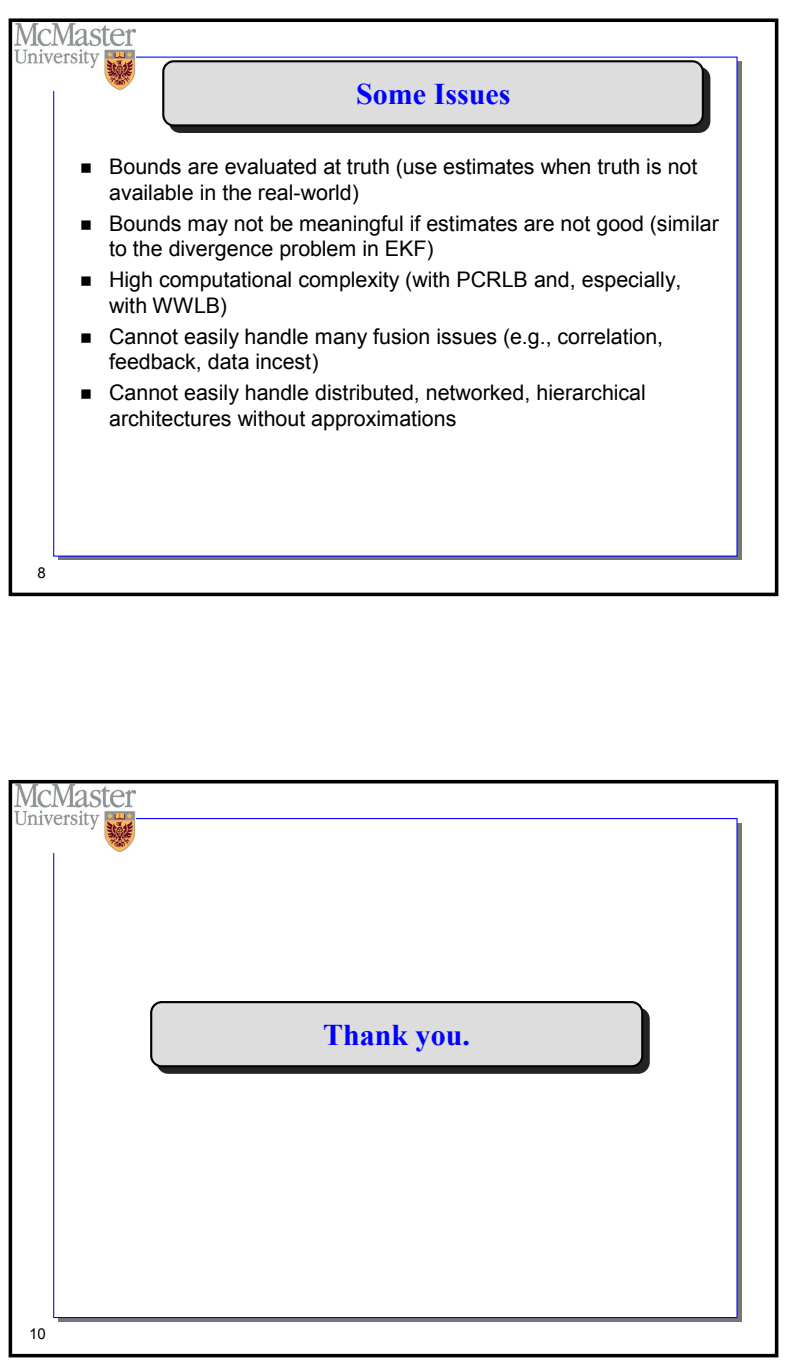


\title{
Issues and Challenges in Performance Assessment of Multitarget Tracking Algorithms with Applications to Real-World Problems: How Useful Are the Theoretical Bounds in the Real-World?
}

\author{
T. Kirubarajan \\ Estimation, Tracking and Fusion Research Laboratory \\ Electrical and Computer Engineering Department, McMaster University \\ Hamilton, Ontario, Canada \\ kiruba@mcmaster.ca
}

\begin{abstract}
A number of performance bounds like, for example, Cramer-Rao Lower Bound and Weiss-Weinstein Bound, have been derived to quantify the performance achievable by any algorithm in state estimation problems. These bounds are evaluated at the ground truth, which means that the exact bound is available only for simulation-based or instrumented real experiments. Also, a number of assumptions on the statistics, state models and their evolution over time are made in order to facilitate the derivation of these complex bounds. As a result, these bounds are at best approximations of achievable performance. In addition, these bounds are often computationally cumbersome, if at all possible, to evaluate in realistic multisensor-multitarget tracking problems. In spite of these limitations, the bounds have been used successfully for performance prediction, tracker evaluation and sensor resource management. In this short paper, we briefly discuss the usefulness of theoretical bounds as realistic performance evaluation measures in real-world tracking problems.
\end{abstract}

Keywords: Multisensor-multitarget tracking, estimation algorithm, performance evaluation, performance bounds, Cramer-Rao lower bound, Weiss-Weinstein bound

\section{INTRODUCTION}

Multisensor-multitarget tracking in the real-world involves a multitude of aspects like, for example, sensor calibration, signal processing, data association, state estimation, performance evaluation, sensor resource management and computational issues [1][2]. That is, tracking is not synonymous with state estimation, but rather it subsumes the latter. A critical, but often neglected, component in tracking is performance evaluation. While it is easy to make a tracker work well and look good on a particular tracking problem and on a particular scenario, it is challenging to make it work uniformly well on most tracking problems. In view of this, there exists an urgent need not only to specify meaningful performance metrics, but also a complete performance evaluation framework with benchmark scenarios. As in many other fields of research, in order to address the issue of impartial evaluation of trackers, different benchmark problems have been proposed for tracking problems as well. In spite of their limited scope, these benchmark problems have been very effective in comparing and contrasting different tracking algorithms in terms of their tracking accuracy, computational complexity and other aspects like sensor usage.

Another common way to evaluate trackers has been the use of theoretical performance metrics like lower bounds on state estimation errors [4]-[9]. These lower bounds quantify the performance achievable by any tracker on a particular scenario. Some of the commonly used theoretical bounds include Cramer-Rao lower bound (CRLB), Weiss-Weinstein lower bound (WWLB) and Bhattachrya bound. These bounds can be calculated off-line, even before the first measurement is received. Also, they are independent of particular measurement values and track estimates. That is, the underlying tracker mechanism does not affect the bound. These properties make the bounds attractive in tracking problems as a way to quantify expected tracking performance. On the other hand, there are a number of shortcomings that may render the bounds useless in realistic tracking problems with real-world issues [3]-[9]. For example, theoretical bounds are to be evaluated at true values of target state, which is often not available in real-world problems. The use of estimated values does not result in meaningful performance limits. In the following, we discuss the practical value of these bounds in realistic target tracking problems. 


\section{TARGET TRACKING IN THE REAL-WORLD}

While the classical single-target-in-a-clean-environment scenario is common in many academic publications, target tracking in the real-world consists of a number of challenging issues. Some of these issues are listed below:

- Multiple heterogeneous targets

- Multiple heterogeneous sensors

- Distributed, hierarchical, networked-systems

- Kinematic and non-kinematic data

- $\quad$ Target birth, spawning, death (time-varying)

- Target maneuvers, time-varying models

- Data association uncertainties

- Bias, location uncertainties, non-Gaussian noise

- Data feedback, fusion, data incest, correlation

- Communication issues, bandwidth limitations

- Data access privileges, reporting responsibilities

- Sensor management issues

- Lack of ground truth, false ground truth

- Fault tolerance, redundancy

- Data of non-quantifiable accuracy (e.g., HUMINT)

While it must be admitted that the theoretical bounds do not consider many of these issues, they serve the following useful purposes in tracking problems:

- They provide an objective (sometimes subjective) achievable performance limit in (at least, simple) tracking problems.

- They can serve as a baseline performance evaluation mechanism before trying the algorithms on more complex scenarios.

- They are useful for "what-if" calculations before deploying sensor assets.

- They provide a measure of performance (MOP) for resource management strategies.

- They are a measure of confidence in the absence of filter calculated values.

- They are a tool for testing trackers, estimates and covariances.

\section{SOME ISSUES AND CHALLENGES}

In spite of their effective use in many target tracking and fusion problems, theoretical performance bounds have some limitations that restrict their applicability to general tracking problems. We list some of those limitations below:

- Bounds are evaluated at truth, and estimates are used when truth is not available in the real-world.

- Bounds may not be meaningful if estimates are not good (similar to the divergence problem in EKF).

- Calculations of these bounds involved high computational complexity (with PCRLB and, especially, with WWLB).

- They cannot easily handle many fusion issues (e.g., correlation, feedback, data incest).

- They cannot easily handle distributed, networked, hierarchical architectures without approximations. 
In view of these limitations, we suggest the following as possible challenges that need to be overcome in order to make theoretical performance bounds useful in realistic multisensor-multitarget tracking problems:

- Bounds that are computationally efficient in large-scale real-world tracking problems.

- Bounds that are effective with long-term prediction and track estimates.

- Bounds that can handle discrete and continuous uncertainties efficiently

- Bounds for common fusion architectures

- Bounds for higher levels of fusion (e.g., classification, intent)

- Bounds for common data association mechanisms (e.g., assignment, multiframe)

- Bounds that are understandable and useful for operators

- Breaking points of bounds.

\section{REFERENCES}

[1] Y. Bar-Shalom and X. R. Li, Multitarget-Multisensor Tracking: Principles and Techniques, YBS Publishing, Storrs, CT, 1995.

[2] Y. Bar-Shalom, X. Li and T. Kirubarajan, Estimation with Applications to Tracking and Navigation, Wiley, New York, 2001.

[3] M. L. Hernandez, T. Kirubarajan and Y. Bar-Shalom, "Multisensor Resource Deployment using Posterior CramerRao bounds”, IEEE Transactions on Aerospace and Electronic Systems, vol. 40, no. 2, pp. 399-416, April 2004.

[4] C. Hue, J.-P. Le Cadre and P. Prez, "Performance Analysis of Two Sequential Monte Carlo Methods and Posterior Cramer-Rao bounds for Multitarget Tracking", Proceedings of the Fifth International Conference on Information Fusion, vol. 1, pp. 464-473, Annapolis, MD, July 2002.

[5] S. Reece and D. Nicholson, "Tighter Alternatives to the Cramer-Rao Lower Bound for Discrete-Time Filtering”, Proc. IEEE Conf. on Decision and Control, Vol. 1, pp. 101-106, Philadelphia, PA, USA, Jul. 2005.

[6] P. Tichavsky, C. S. Muravchik, and A. Nehorai, "Posterior Cramer-Rao Bounds for Discrete-Time Nonlinear Filtering”, IEEE Trans. Signal Processing, Vol. 46, pp. 1386--1396, May 1998.

[7] E. Weinstein and A. J. Weiss, “A General Class of Lower Bounds in Parameter Estimation”, IEEE Trans. Information Theory, Vol. 34, pp. 338--342, Mar. 1988.

[8] A. J. Weiss and E. Weinstein, “A Lower Bound on the Mean Square Error in Random Parameter Estimation”, IEEE Trans. Information Theory, Vol. IT-31, pp. 680--682, Sep. 1985.

[9] X. Zhang and P. K. Willett, "Cramer-Rao bounds for Discrete Time Linear Filtering with Measurement Origin Uncertainty", Proceedings of the Workshop on Estimation, Tracking and Fusion: A Tribute to Yaakov Bar-Shalom, pp. 546-561, Monterey, CA, May 2001. 